\title{
AN ERROR ESTIMATE FOR A FINITE-VOLUME SCHEME FOR THE CAHN-HILLIARD EQUATION WITH DYNAMIC BOUNDARY CONDITIONS
}

\author{
FLORE NABET*
}

\begin{abstract}
In this paper we consider a finite-volume approximation for the Cahn-Hilliard equation with dynamic boundary conditions. The convergence of the scheme is proved in [22], we prove here an error estimate for the fully-discrete scheme. We also give numerical simulations which validate the theoretical result.
\end{abstract}

Key words. Cahn-Hilliard equation, dynamic boundary condition, finite-volume method, error estimate.

AMS subject classifications. 35K55, 65M08, 65M15, 76M12

\section{Introduction.}

1.1. The Cahn-Hilliard model. We consider the following Cahn-Hilliard equation with a dynamic boundary condition which describes the phase separation process of a binary mixture. Find the concentration of one of the two components $c:(0, T) \times \Omega \rightarrow \mathbb{R}$ and the chemical potential $\mu:(0, T) \times \Omega \rightarrow \mathbb{R}$ such that

$$
\begin{aligned}
\partial_{t} c & =\Gamma_{b} \Delta \mu ; & & \text { in }(0, T) \times \Omega ; \\
\mu & =-\frac{3}{2} \varepsilon \sigma_{b} \Delta c+\frac{12}{\varepsilon} \sigma_{b} f_{b}^{\prime}(c) ; & & \text { in }(0, T) \times \Omega ; \\
\frac{\varepsilon^{3}}{64 \Gamma_{b} \Gamma_{s} \partial_{t} c_{\mid \Gamma}} & =\frac{3}{8} \varepsilon^{2} \sigma_{b} \sigma_{s} \Delta_{\Gamma} c_{\mid \Gamma}-6 \sigma_{b} f_{s}^{\prime}\left(c_{\mid \Gamma}\right)-\frac{3}{2} \varepsilon \sigma_{b} \partial_{n} c ; & & \text { on }(0, T) \times \Gamma ; \\
\partial_{n} \mu & =0 ; & & \text { on }(0, T) \times \Gamma ; \\
c(0, .) & =c^{0} ; & & \text { in } \Omega ;
\end{aligned}
$$

where $\Omega$ is a connected and bounded domain of class $\mathcal{C}^{2}$ in $\mathbb{R}^{2}, \partial_{n}$ is the normal derivative operator, $c_{\Gamma \Gamma}$ is the trace of $c$ on the boundary $\Gamma=\partial \Omega$ and $\Delta_{\Gamma}$ is the Laplace-Beltrami operator.

The Cahn-Hilliard model is a diffuse interface model that means that the interfaces have a small but non-zero thickness $\varepsilon>0$ (see Fig. 1a). Several physical parameters which describe the physical properties of the mixture components and the wall appear in the model: a diffusion coefficient called the mobility (supposed to be constant here) $\Gamma_{b}>0$, the binary surface tension coefficient $\sigma_{b}>0$ between the two components (which is the density interfacial energy), a capillarity coefficient $\sigma_{s}>0$ and a relaxation coefficient $\Gamma_{s}>0$. The bulk and surface potentials $f_{b}$ (typically $f_{b}(c)=c^{2}(1-c)^{2}$, see Fig. 1b) and $f_{s}$ respectively satisfy the following dissipativity assumption (useful to prove the bounds on the discrete solutions given in Proposition 4.5),

$$
\liminf _{|c| \rightarrow \infty} f_{b}^{\prime \prime}(c)>0 \text { and } \quad \liminf _{|c| \rightarrow \infty} f_{s}^{\prime \prime}(c)>0,
$$

and the polynomial growth condition for $f_{b}$

$$
\left|f_{b}^{\prime}(c)\right| \leq C\left(1+|c|^{p}\right), \quad \forall c \in \mathbb{R}
$$

for some $C>0$ and $p \in[1,+\infty[$.

${ }^{*}$ CMAP, Ecole polytechnique, CNRS, Université Paris-Saclay, 91128, Palaiseau, France (flore.nabet@polytechnique.edu). 


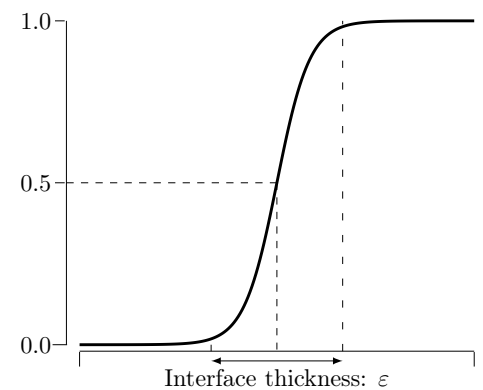

(a) Interface thickness

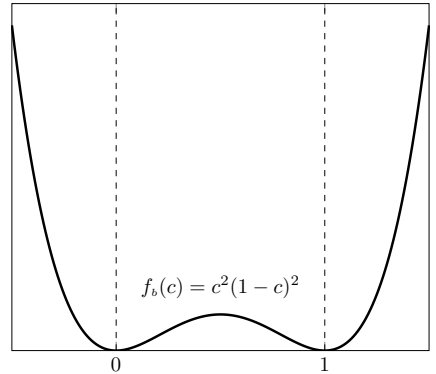

(b) Bulk potential

Fig. 1: Definition of the interface thickness and double-well structure of $f_{b}$

The total Cahn-Hilliard energy is written as follows

$$
\mathcal{F}(c)=\underbrace{\int_{\Omega}\left(\frac{3}{4} \varepsilon \sigma_{b}|\nabla c|^{2}+\frac{12}{\varepsilon} \sigma_{b} f_{b}(c)\right)}_{:=\mathcal{F}_{b}(c)}+\underbrace{\int_{\Gamma}\left(\frac{3}{16} \varepsilon^{2} \sigma_{b} \sigma_{s}\left|\nabla_{\Gamma} c_{\mid \Gamma}\right|^{2}+6 \sigma_{b} f_{s}\left(c_{\mid \Gamma}\right)\right)}_{:=\mathcal{F}_{s}(c)},
$$

and this energy is dissipated over time

$$
\frac{\mathrm{d}}{\mathrm{d} t} \mathcal{F}(c(t, .))=-\Gamma_{b} \int_{\Omega}|\nabla \mu(t, .)|^{2}-\frac{\varepsilon^{3}}{64 \Gamma_{b} \Gamma_{s}} \int_{\Gamma}\left|\partial_{t} c_{1 \Gamma}(t, .)\right|^{2}, \quad t \in[0, T[.
$$

We can remark that the bulk energy $\mathcal{F}_{b}$ is the energy associated with the Cahn-Hilliard equation with Neumann boundary conditions. Definition (1.3) of the total energy induces us to introduce the function spaces $H_{\Gamma}^{1}(\Omega)=\left\{u \in H^{1}(\Omega): u_{\mid \Gamma} \in H^{1}(\Gamma)\right\}$ and $H_{\Gamma}^{2}(\Omega)=\left\{u \in H^{2}(\Omega): u_{\mid \Gamma} \in\right.$ $\left.H^{2}(\Gamma)\right\}$ and the corresponding norms, for $i \in\{1,2\}$

$$
\|u\|_{H_{\Gamma}^{i}(\Omega)}=\left(\|u\|_{H^{i}(\Omega)}^{2}+\left\|u_{1 \Gamma}\right\|_{H^{i}(\Gamma)}^{2}\right)^{\frac{1}{2}}, \forall u \in H_{\Gamma}^{i}(\Omega) .
$$

In the analysis to follow, for the sake of simplicity, all the coefficients in problem (1.1) will be taken equal to one (expect for the numerical results given in Section 5).

1.2. Former results and outline. In the past 30 years, the Cahn-Hilliard equation associated with the homogeneous Neumann boundary condition on the order parameter $c$ has been extensively studied. Recently physicists $[15,16,20]$ have introduced the dynamic boundary condition which allows to take into account the interaction between the components and the wall, especially the contact-line dynamics (see [18]). In the case of Neumann boundary conditions the numerical analysis with finite-difference and finite-element methods is wellunderstood (see $[3,5,6,7,9,8,10,12,13,14,17,23]$ and the references therein). However, to our knowledge, for the problem that we study here there is no error estimate for the fullydiscrete scheme on a curved domain. The only work on the numerical analysis of parabolic problems with dynamic boundary conditions on a smooth domain that we know is given in [21]. The authors prove error estimates for several parabolic problems, including semilinear problems such as Allen-Cahn equation, with dynamic boundary conditions on a $\mathcal{C}^{\infty}$ domain using finiteelement discretization in space. With regard to the Cahn-Hilliard equation, there exist finitedifference methods but without proof of stability or convergence (see $[15,16,20]$ ). A numerical 
analysis for the semi-discrete scheme using a spatial finite-element scheme is done in [2] in a slab with periodic boundary conditions in the longitudinal direction. In [22] the author propose a finite-volume scheme and prove the convergence of the numerical scheme towards a weak solution of problem (1.1) for a smooth non-polygonal domain. Finite-volume methods have advantage to easily adapt to the non flat geometry of the boundary and to naturally couple the equation in the domain and the dynamic boundary condition by the flux term $\partial_{n} c$. That is why, in order to prove an error estimate on a smooth domain $\Omega$ for this problem, we use the same finite-volume scheme that the one introduced in [22]. Therefore in Section 2 we present the finite-volume framework that is the finite-volume notation on a curved domain, the associated discrete inner products and norms and the functional inequalities used in the paper. Then Section 3 is devoted to the presentation of the numerical scheme. In Section 4 (and in the Appendix) we prove the main result of the paper: an error estimate for the fully-discrete scheme (Theorem 4.2). Finally in Section 5 we present a numerical error estimate for this model in accordance with the error estimate theorem proved in the previous section. Note that the paper is written in the two dimensional case. If we want to study the three dimensional case, which would be a natural perspective for this work, the main difficulty is the handling of the Laplace-Beltrami operator in a two-dimensional surface.

\section{The finite-volume framework.}

2.1. Mesh and notation. We recall here the main finite-volume notations used in the paper (see Fig. 2). The usual notation for a polygonal domain can be found for example in [11] and the notation associated to a curved domain in [22].
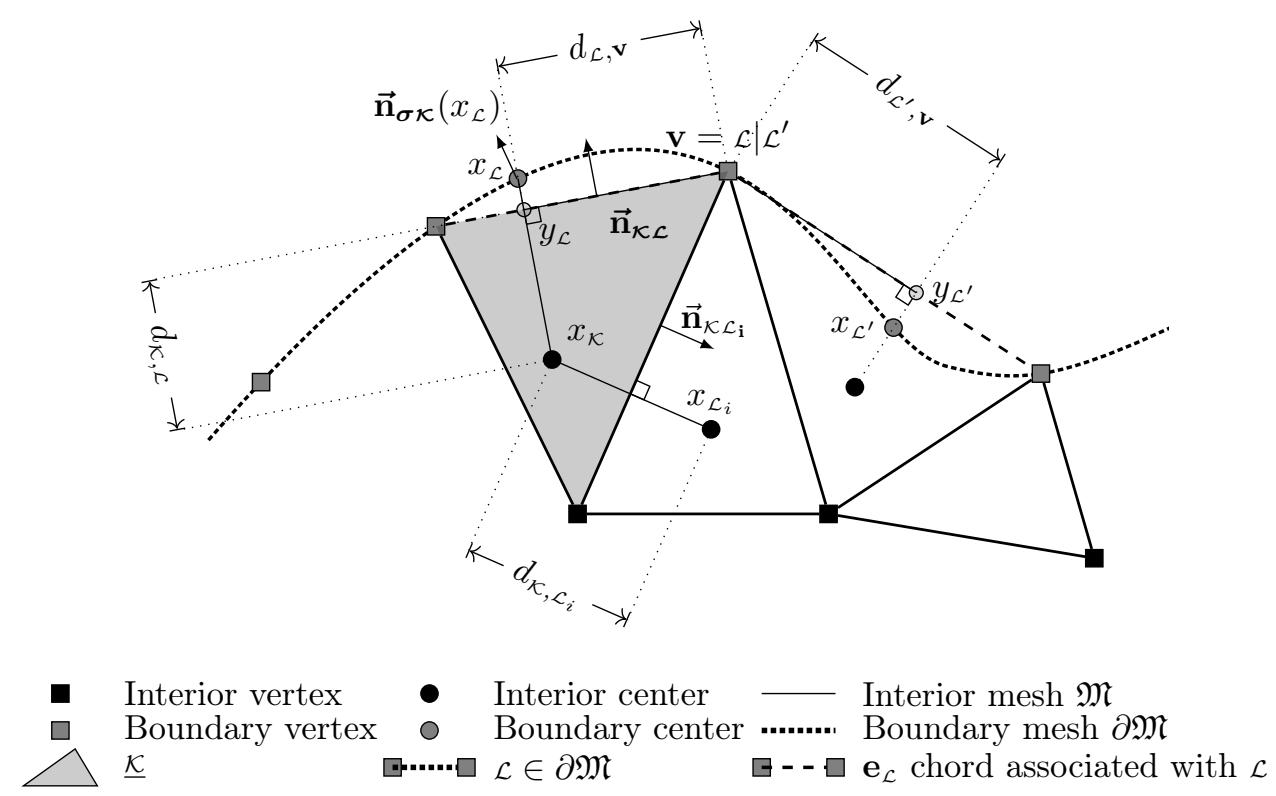

Fig. 2: Mesh $\mathcal{T}$ associated with $\Omega$

An admissible mesh $\mathcal{T}$ of $\Omega$ is given by an interior mesh $\mathfrak{M}$ and a boundary mesh $\partial \mathfrak{M}$. The interior mesh $\mathfrak{M}$ is a set of disjoint open subsets of $\Omega$, denoted by $\mathcal{K}$ and called interior control volumes, which satisfy:

- $\bar{\Omega}=\cup_{\mathcal{K} \in \mathfrak{M} \overline{\mathcal{K}} ;}$ 
- if $\mathcal{K}, \mathcal{L} \in \mathfrak{M}, \mathcal{K} \neq \mathcal{L}$, then $\mathcal{K} \cap \mathcal{L}=\emptyset$;

- if $\mathcal{K}, \mathcal{L} \in \mathfrak{M}, \mathcal{K} \neq \mathcal{L}$ such that the dimension of $\overline{\mathcal{K}} \cap \overline{\mathcal{L}}$ is equal to 1 , then $\overline{\mathcal{K}} \cap \overline{\mathcal{L}}$ is the edge of the mesh separating the control volumes $\mathcal{K}$ and $\mathcal{L}$;

- if $\mathcal{K} \cap \Gamma$ contains a finite number of points, then $\mathcal{K}$ is polygonal;

- for any $\mathcal{K} \in \mathfrak{M}$, we associate a point $x_{\mathcal{K}} \in \mathcal{K}$ (referred to as the center of $\mathcal{K}$ ) such that if $\mathcal{K}, \mathcal{L}$ are two neighboring interior control volumes, the edge which separates $\mathcal{K}$ and $\mathcal{L}$, which is denoted by $\sigma=\mathcal{K} \mid \mathcal{L}$, is orthogonal to the straight line going through $x_{\mathcal{K}}$ and $x_{\mathcal{L}}$.

Let $\mathcal{E}$ be the set of edges of the interior mesh $\mathfrak{M}$. We decompose $\mathcal{E}$ into two disjoint subsets: the set of interior (flat) edges $\mathcal{E}_{\text {int }}=\{\sigma \in \mathcal{E}: \sigma \not \subset \Gamma\}$ and the set of exterior (curved) edges $\mathcal{E}_{\text {ext }}=\{\sigma \in \mathcal{E}: \sigma \subset \Gamma\}$. Similarly we use the notations $\mathcal{E}_{\mathcal{K}}^{\text {int }}$ and $\mathcal{E}_{\mathcal{K}}^{\text {ext }}$ for the edges of a given control volume $\mathcal{K} \in \mathfrak{M}$. For any $\sigma \in \mathcal{E}$, we note $m_{\sigma}$ its length. For each edge $\sigma \in \mathcal{E}$, we associate a diamond cell $\mathcal{D}$ defined as follows:

- $\mathcal{D}=\mathcal{D}_{\sigma}$ the quadrangle whose diagonals are the edge $\sigma$ and the line segment $\left[x_{\mathcal{K}}, x_{\mathcal{L}}\right]$ if $\sigma \in \mathcal{E}_{\text {int }}$;

- $\mathcal{D}=\mathcal{D}_{\sigma}=\left\{t x+(1-t) x_{\mathcal{K}}, t \in[0,1], x \in \sigma\right\}$ if $\sigma \in \mathcal{E}_{\text {ext }} \cap \mathcal{E}_{\mathcal{K}}$.

We note $m_{\mathcal{D}}$ the Lebesgue measure of $\mathcal{D}$ and $\mathfrak{D}$ is the set of all diamond cells.

Since the domain $\Omega$ is not polygonal, we have to introduce an approximate domain $\underline{\Omega}=$

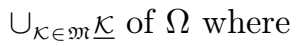

- $\underline{\mathcal{K}}=\mathcal{K}$ if $\mathcal{E}_{\mathcal{K}} \cap \mathcal{E}_{\text {ext }}=\emptyset$;

- $\underline{\mathcal{K}}$ is the polygon obtained by joining all the vertex of $\mathcal{K}$ if $\mathcal{E}_{\mathcal{K}} \cap \mathcal{E}_{\text {ext }} \neq \emptyset$. We can notice that in this case $\mathcal{K}$ may be not convex and that $\underline{\mathcal{K}}$ may be not included in $\Omega$.

We denote by $m_{\mathcal{K}}\left(\right.$ resp. $\left.m_{\underline{\mathcal{K}}}\right)$ the Lebesgue measure of $\mathcal{K}$ (resp. $\underline{\mathcal{K}}$ ) and by $m_{\mathfrak{M}} \in \mathbb{R}^{\mathfrak{M}}$ (resp. $\left.m_{\mathfrak{M}} \in \mathbb{R}^{\mathfrak{M}}\right)$ the vector $\left(m_{\mathcal{K}}\right)_{\mathcal{K} \in \mathfrak{M}}\left(\right.$ resp. $\left.\left(m_{\underline{\mathcal{K}}}\right)_{\mathcal{K} \in \mathfrak{M}}\right)$. The Lebesgue measures of $\mathcal{K}$ and $\underline{\mathcal{K}}$ are then related by the following relation.

Proposition 2.1. For any interior control volume $\mathcal{K} \in \mathfrak{M}$ such that the dimension of $\partial \mathcal{K} \cap \Gamma$ is equal to 1 , we have

$$
m_{\underline{\mathcal{K}}}-m_{\mathcal{K}}=\mathcal{O}\left(\operatorname{diam}(\mathcal{K})^{3}\right) .
$$

Therefore, there exists $C_{1}, C_{2}>0$ depending only on $\Gamma$ such that

$$
C_{1} m_{\underline{\mathcal{K}}} \leq m_{\mathcal{K}} \leq C_{2} m_{\underline{\mathcal{K}}} .
$$

The boundary mesh $\partial \mathfrak{M}$ is equal to the set of exterior edges $\mathcal{E}_{\text {ext }}$. Thus, the exterior edges are also boundary control volumes. When we consider them as edges, we denote them by $\sigma \in \mathcal{E}_{\text {ext }}$, and, when we consider them as control volumes of the boundary mesh, we denote them by $\mathcal{L} \in \partial \mathfrak{M}$ (and its length by $m_{\mathcal{L}}$ ). The chord associated with $\mathcal{L}$ is then denoted by $\mathbf{e}_{\mathcal{L}}$ (and its length by $m_{\mathbf{e}_{\mathcal{L}}}$ ), and, the quantities $m_{\mathcal{L}}$ and $m_{\mathbf{e}_{\mathcal{L}}}$ are related by the following relation.

Proposition 2.2. For any boundary control volume $\mathcal{L} \in \partial \mathfrak{M}$, we have

$$
m_{\mathbf{e}_{\mathcal{L}}}-m_{\mathcal{L}}=\mathcal{O}\left(m_{\mathcal{L}}^{3}\right) .
$$

In particular, there exists $C_{3}>0$ independent of $\operatorname{size}(\mathcal{T})$ such that

$$
m_{\mathbf{e}_{\mathcal{L}}} \leq m_{\mathcal{L}} \leq C_{3} m_{\mathbf{e}_{\mathcal{L}}} .
$$

Let $m_{\partial \mathfrak{M}}$ (resp. $\left.m_{\underline{\partial \mathfrak{M}}}\right)$ be the vector $\left(m_{\mathcal{L}}\right)_{\mathcal{L} \in \partial \mathfrak{M}}$ (resp. $\left.\left(m_{\mathbf{e}_{\mathcal{L}}}\right)_{\mathcal{L} \in \partial \mathfrak{M}}\right)$, then we note $m_{\mathcal{T}}=$ $\left(m_{\mathfrak{M}}, m_{\partial \mathfrak{M}}\right) \in \mathbb{R}^{\mathcal{T}}\left(\operatorname{resp} . m_{\mathcal{I}}=\left(m_{\underline{\mathfrak{M}}}, m_{\underline{\partial \mathfrak{M}}}\right) \in \mathbb{R}^{\mathcal{T}}\right)$.

For any control volume $\mathcal{L} \in \overline{\partial M}$, we associate a point $x_{\mathcal{L}} \in \mathcal{L}$, called the center of the control volume. For any boundary control volume $\mathcal{L} \in \partial \mathfrak{M}$, let be $\mathcal{K} \in \mathfrak{M}$ the interior control 
volume such that $\mathcal{L}=\sigma$ is an edge of $\mathcal{K}$. Then, we impose that the straight line going through $x_{\mathcal{K}}$ and $x_{\mathcal{L}}$ is orthogonal to the chord $\mathbf{e}_{\mathcal{L}}$ associated with $\mathcal{L}$. Moreover, we define $y_{\mathcal{L}}$ as the orthogonal projection of $x_{\mathcal{L}}$ on the chord $\mathbf{e}_{\mathcal{L}}$.

Let $\mathcal{V}$ be the set of vertices of the mesh $\mathfrak{M}$ which belongs to $\Gamma$. We denote by $\mathbf{v}=\mathcal{L} \mid \mathcal{L}^{\prime}$ the vertex which separates the boundary control volumes $\mathcal{L}$ and $\mathcal{L}^{\prime}$. For any $\mathbf{v}=\mathcal{L} \mid \mathcal{L}^{\prime}$, let $d_{\mathcal{L}, \mathbf{v}}=d\left(y_{\mathcal{L}}, \mathbf{v}\right)$ be the approximation of the length $m_{\gamma_{\mathcal{L} \mathbf{v}}}$ of the $\operatorname{arc} \gamma_{\mathcal{L} \mathbf{v}}$ included in the boundary control volume $\mathcal{L}$ whose ends are $x_{\mathcal{L}}$ and $\mathbf{v}$. The measure of the $\operatorname{arc} \gamma_{\mathcal{L L}^{\prime}}$ (which is the arc whose ends are $x_{\mathcal{L}}$ and $x_{\mathcal{L}^{\prime}}$ and passing through the vertex $\mathbf{v}$ ) is then approximate by the distance $d_{\mathcal{L}, \mathcal{L}^{\prime}}=d_{\mathcal{L}, \mathbf{v}}+d_{\mathcal{L}^{\prime}, \mathbf{v}}$

If $\sigma=\mathcal{K} \mid \mathcal{L} \in \mathcal{E}_{\text {int }}$ is an interior edge, we note $d_{\mathcal{K}, \mathcal{L}}$ the distance between the centers $x_{\mathcal{K}}$ and $x_{\mathcal{L}}$; and $\overrightarrow{\mathbf{n}}_{\mathcal{K} \mathcal{L}}$ the normal vector to $\sigma$ going from $\mathcal{K}$ to $\mathcal{L}$. If $\sigma=\mathcal{L} \in \mathcal{E}_{\text {ext }} \cap \mathcal{E}_{\mathcal{K}}$ is an exterior edge, we note $d_{\mathcal{K}, \mathcal{L}}$ the distance between the center $x_{\mathcal{K}}$ and the point $y_{\mathcal{L}}$; and $\overrightarrow{\mathbf{n}}_{\mathcal{K} \mathcal{L}}$ the normal vector to the chord $\mathbf{e}_{\mathcal{L}}$ outward to $\mathcal{K}$. In this case, the distance $d\left(x_{\mathcal{K}}, x_{\mathcal{L}}\right)$ and its approximation $d_{\mathcal{K}, \mathcal{L}}$ satisfy the following relation.

Proposition 2.3. Let us consider a boundary control volume $\mathcal{L} \in \partial \mathfrak{M}$ such that $\mathcal{L}=\sigma \in$ $\mathcal{E}_{\mathcal{K}}$ where $\mathcal{K} \in \mathfrak{M}$ is an interior control volume, then we have

$$
d\left(x_{\mathcal{L}}, y_{\mathcal{L}}\right)=\left|d\left(x_{\mathcal{K}}, x_{\mathcal{L}}\right)-d_{\mathcal{K}, \mathcal{L}}\right|=\mathcal{O}\left(m_{\mathcal{L}} m_{\gamma_{\mathcal{L} \mathbf{v}}}\right) .
$$

Let $\operatorname{size}(\mathcal{T})$ be the maximum of the diameters of the interior control volumes $\mathcal{K}$. We introduce a positive number $\operatorname{reg}(\mathcal{T})$ that measures the regularity of a given mesh and is useful to perform the numerical analysis of finite-volume schemes

$$
\operatorname{reg}(\mathcal{T}):=\max \left(\max _{\mathcal{K} \in \mathfrak{M}} \frac{\operatorname{diam}(\mathcal{K})}{\sqrt{m_{\mathcal{K}}}}, \max _{\substack{\mathcal{K} \in \mathfrak{M} \\ \sigma \in \mathcal{E}_{\mathcal{K}}}} \frac{\operatorname{diam}(\mathcal{K})}{d\left(x_{\mathcal{K}}, \sigma\right)}, \max _{\substack{\mathcal{K} \in \mathfrak{M} \\ \sigma \in \mathcal{E}_{\mathcal{K}}}} \frac{d_{\mathcal{K}, \mathcal{L}}}{d\left(x_{\mathcal{K}}, \sigma\right)}\right) .
$$

The number $\operatorname{reg}(\mathcal{T})$ should be uniformly bounded when $\operatorname{size}(\mathcal{T}) \rightarrow 0$.

For the needs of the proof of error estimate theorem, we also define a family of quasiuniform meshes.

DEFINITION 2.4 (Quasi-uniform mesh family of $\Omega$ ).

For a given mesh $\mathcal{T}$, we define the number $\operatorname{reg}_{\text {unif }}(\mathcal{T})$ as follows

$$
\operatorname{reg}_{\text {unif }}(\mathcal{T}) \stackrel{\text { def }}{=} \sup \left(\operatorname{reg}(\mathcal{T}), \sup _{\mathcal{K} \in \mathfrak{M}} \frac{\operatorname{size}(\mathcal{T})^{2}}{m_{\mathcal{K}}}\right) .
$$

We say that a mesh family $\left(\mathcal{T}^{(i)}\right)_{i \in \mathbb{N}}$ is quasi-uniform if $\operatorname{reg}_{\text {unif }}\left(\mathcal{T}^{(i)}\right)$ is bounded.

2.2. Inner-products and norms. Since the domain $\Omega$ is not polygonal, we introduce a $L^{2}$-inner product on the domain $\Omega$ (and on its boundary $\Gamma=\partial \Omega$ ) but also on the approximate polygonal domain $\underline{\Omega}$ (and on its boundary $\partial \underline{\Omega}$ ).

For the space discretization, the finite-volume method associates an unknown value $u_{\mathcal{K}} \in \mathbb{R}$ (resp. $u_{\mathcal{L}} \in \mathbb{R}$ ) to each interior (resp. boundary) control volume $\mathcal{K} \in \mathfrak{M}$ (resp. $\left.\mathcal{L} \in \partial \mathfrak{M}\right)$. Thus we note

$$
u_{\mathcal{T}}=\left(u_{\mathfrak{M}}, u_{\partial \mathfrak{M}}\right)=\left(\left(u_{\mathcal{K}}\right)_{\mathcal{K} \in \mathfrak{M}},\left(u_{\mathcal{L}}\right)_{\mathcal{L} \in \partial \mathfrak{M}}\right) \in \mathbb{R}^{\mathcal{T}}=\mathbb{R}^{\mathfrak{M}} \times \mathbb{R}^{\partial \mathfrak{M}} .
$$

Definition 2.5 (Discrete $L^{2}$-inner products).

- We define the inner product $(., .)_{L^{2}(\Omega)}$ on $L^{2}(\Omega)$ and the inner product $(., .)_{\mathfrak{M}}$ on $L^{2}(\underline{\Omega})$ as follows: for any $u_{\mathfrak{M}}, v_{\mathfrak{M}} \in \mathbb{R}^{\mathfrak{M}}$, we have

$$
\left(u_{\mathfrak{M}}, v_{\mathfrak{M}}\right)_{L^{2}(\Omega)}=\sum_{\mathcal{K} \in \mathfrak{M}} m_{\mathcal{K}} u_{\mathcal{K}} v_{\mathcal{K}} \quad \text { and } \quad\left(u_{\mathfrak{M}}, v_{\mathfrak{M}}\right)_{\mathfrak{M}}=\sum_{\mathcal{K} \in \mathfrak{M}} m_{\underline{\mathcal{K}}} u_{\mathcal{K}} v_{\mathcal{K}}
$$


We denote by $\|\cdot\|_{L^{2}(\Omega)}$ and $\|\cdot\|_{0, \mathfrak{M}}$ the associated norms.

- We define the inner product $(., .)_{L^{2}(\Gamma)}$ on $L^{2}(\Gamma)$ and the inner product $(., .)_{\partial \mathfrak{M}}$ on $L^{2}(\partial \underline{\Omega})$ as follows: for any $u_{\partial \mathfrak{M}}, v_{\partial \mathfrak{M}} \in \mathbb{R}^{\partial \mathfrak{M}}$, one has

$$
\left(u_{\partial \mathfrak{M}}, v_{\partial \mathfrak{M}}\right)_{L^{2}(\Gamma)}=\sum_{\mathcal{L} \in \partial \mathfrak{M}} m_{\mathcal{L}} u_{\mathcal{L}} v_{\mathcal{L}} \text { and }\left(u_{\partial \mathfrak{M}}, v_{\partial \mathfrak{M}}\right)_{\partial \mathfrak{M}}=\sum_{\mathcal{L} \in \partial \mathfrak{M}} m_{\mathbf{e}_{\mathcal{L}}} u_{\mathcal{L}} v_{\mathcal{L}}
$$

We denote by $\|\cdot\|_{L^{2}(\Gamma)}$ and $\|\cdot\|_{0, \partial m}$ the associated norms.

Owing to Proposition 2.1 (resp. Proposition 2.2), the norms $\|\cdot\|_{L^{2}(\Omega)}$ and $\|\cdot\|_{0, \mathfrak{M}}$ (resp. $\|\cdot\|_{L^{2}(\Gamma)}$ and $\left.\|\cdot\|_{0, \partial \mathfrak{M}}\right)$ are equivalent (with constants independent on the mesh size).

We also define semi-inner products in $\mathbb{R}^{\mathcal{T}}$ and $\mathbb{R}^{2 \mathfrak{M}}$.

Definition 2.6 (Discrete $H^{1}$-semidefinite inner products). We define the $H^{1}$-semidefinite inner product in $\mathbb{R}^{\mathcal{T}}$ as follows: for any $u_{\mathcal{T}}, v_{\mathcal{T}} \in \mathbb{R}^{\mathcal{T}}$,

$$
\llbracket u_{\mathcal{T}}, v_{\mathcal{T}} \rrbracket_{1, \mathcal{T}}=\sum_{\sigma=\mathcal{K} \mid \mathcal{L} \in \mathcal{E}_{i n t}} \frac{m_{\sigma}}{d_{\mathcal{K}, \mathcal{L}}}\left(u_{\mathcal{K}}-u_{\mathcal{L}}\right)\left(v_{\mathcal{K}}-v_{\mathcal{L}}\right)+\sum_{\sigma=\mathcal{L} \in \mathcal{E}_{\text {ext }}} \frac{m_{\mathbf{e}_{\mathcal{L}}}}{d_{\mathcal{K}, \mathcal{L}}}\left(u_{\mathcal{K}}-u_{\mathcal{L}}\right)\left(v_{\mathcal{K}}-v_{\mathcal{L}}\right) .
$$

We also define the $H^{1}$-semidefinite inner product in $\mathbb{R}^{\partial \mathfrak{M}}$ as follows:

$$
\llbracket u_{\partial \mathfrak{M}}, v_{\partial \mathfrak{M}} \rrbracket_{1, \partial \mathfrak{M}}=\sum_{\mathbf{v}=\mathcal{L} \mid \mathcal{L}^{\prime} \in \mathcal{V}} \frac{1}{d_{\mathcal{L}, \mathcal{L}^{\prime}}}\left(u_{\mathcal{L}}-u_{\mathcal{L}^{\prime}}\right)\left(v_{\mathcal{L}}-v_{\mathcal{L}^{\prime}}\right), \forall u_{\partial \mathfrak{M}}, v_{\partial \mathfrak{M}} \in \mathbb{R}^{\partial \mathfrak{M}} .
$$

We denote by $|\cdot|_{1, \mathcal{T}}$ and $|\cdot|_{1, \partial \mathfrak{m}}$ the associated seminorms. Moreover, we define the $H^{1}$-norms in $\mathbb{R}^{\mathcal{T}}$ and $\mathbb{R}^{\partial \mathfrak{M}}$ as follows: for any $u_{\mathcal{T}} \in \mathbb{R}^{\mathcal{T}}, u_{\partial \mathfrak{M}} \in \mathbb{R}^{\partial \mathfrak{M}}$,

$$
\left\|u_{\mathcal{T}}\right\|_{1, \mathcal{T}}=\left(\left\|u_{\mathcal{T}}\right\|_{0, \mathfrak{M}}^{2}+\left|u_{\mathcal{T}}\right|_{1, \mathcal{T}}^{2}\right)^{\frac{1}{2}} \quad \text { and } \quad\left\|u_{\partial \mathfrak{M}}\right\|_{1, \partial \mathfrak{M}}=\left(\left\|u_{\partial \mathfrak{M}}\right\|_{0, \partial \mathfrak{M}}^{2}+\left|u_{\partial \mathfrak{M}}\right|_{1, \partial \mathfrak{M}}^{2}\right)^{\frac{1}{2}} .
$$

2.3. Functional inequalities. We give here without proofs some functional inequalities available in the literature and that we will use all along the paper. In [1] the proofs are given on a polygonal domain using the continuous embedding of $B V(\Omega)$ into $L^{2}(\Omega)$. Since the properties of the space $B V(\Omega)$ used in [1] hold with a Lipschitz, bounded, connected domain of $\mathbb{R}^{2}$ and that computations performed in [1] are also true for the norms defined in Section 2.2, the results can be adapted in our case. We consider an admissible mesh $\mathcal{T}$ of $\Omega$.

Lemma 2.7 (Discrete mean Poincaré inequality, [1, Theorem 3.6]). There exists $C_{4}>0$ depending only on $\Omega$ such that for any $u_{\mathcal{T}} \in \mathbb{R}^{\mathcal{T}}$,

$$
\left\|u_{\mathfrak{M}}-m_{\Omega}\left(u_{\mathfrak{M}}\right)\right\|_{L^{2}(\Omega)} \leq C_{4}\left|u_{\mathcal{T}}\right|_{1, \mathcal{T}} \text { with } m_{\Omega}\left(u_{\mathfrak{M}}\right)=\frac{1}{|\Omega|} \sum_{\mathcal{K} \in \mathfrak{M}} m_{\mathcal{K}} u_{\mathcal{K}}
$$

Thus, we also have

$$
\left\|u_{\mathfrak{m}}\right\|_{L^{2}(\Omega)} \leq C_{4}\left|u_{\mathcal{T}}\right|_{1, \mathcal{T}}+|\Omega|^{\frac{1}{2}}\left|m_{\Omega}\left(u_{\mathfrak{m}}\right)\right|
$$

Comparing the mean values on $\Omega$ and $\underline{\Omega}$ we also obtain a Poincaré inequality that involves the mean value on $\underline{\Omega}$. More precisely, thanks to Propostion 2.1 and the mesh regularity (2.1), there exists $C_{5}>0$ depending only on $\Gamma$ and $\operatorname{reg}(\mathcal{T})$ such that for any $u_{\mathcal{T}} \in \mathbb{R}^{\mathcal{T}}$,

$$
\left\|u_{\mathfrak{M}}\right\|_{L^{2}(\Omega)} \leq C_{4}\left|u_{\mathcal{T}}\right|_{1, \mathcal{T}}+\frac{|\Omega|^{\frac{1}{2}}}{C_{1}}\left|m_{\underline{\Omega}}\left(u_{\mathfrak{M}}\right)\right|+\frac{C_{5}}{|\Omega|^{\frac{1}{2}}} \operatorname{size}(\mathcal{T})\left\|u_{\mathfrak{M}}\right\|_{L^{1}(\Omega)},
$$

with $m_{\underline{\Omega}}\left(u_{\mathfrak{M}}\right)=\frac{1}{|\underline{\Omega}|} \sum_{\mathcal{K} \in \mathfrak{M}} m_{\underline{\mathcal{K}}} u_{\mathcal{K}}$. 
Lemma 2.8 (Poincaré-Sobolev inequality, [1, Theorem 3.2]). Let $1 \leq q<+\infty$, there exists $C_{6}>0$ depending only on $q, \Omega$ and $\operatorname{reg}(\mathcal{T})$ such that

$$
\left\|u_{\mathfrak{M}}\right\|_{L^{q}(\Omega)}:=\left(\sum_{\mathcal{K} \in \mathfrak{M}} m_{\mathcal{K}}\left|u_{\mathcal{K}}\right|^{q}\right)^{\frac{1}{q}} \leq C_{6}\left\|u_{\mathcal{T}}\right\|_{1, \mathcal{T}}, \quad \forall u_{\mathcal{T}} \in \mathbb{R}^{\mathcal{T}}
$$

We can also easily prove the following Sobolev inequality on the one dimensional manifold $\Gamma$.

Lemma 2.9 ([22, Lemma A.3]). There exists $C_{7}>0$ depending only on $\Gamma$ such that for any $u_{\partial \mathfrak{M}} \in \mathbb{R}^{\partial \mathfrak{M}}$,

$$
\sup _{\mathcal{L} \in \partial \mathfrak{M}}\left|u_{\mathcal{L}}\right| \leq C_{7}\left\|u_{\partial \mathfrak{M}}\right\|_{1, \partial \mathfrak{M}}
$$

Using the fact that the trace operator is continuous from $B V(\Omega)$ into $L^{1}(\Gamma)$, we can use similar techniques as that in [1, Theorem 3.2] to obtain the following discrete trace theorem.

Lemma 2.10 (Trace inequality). There exists $C_{8}>0$ depending only on $\Omega$ such that for any $u_{\mathcal{T}} \in \mathbb{R}^{\mathcal{T}}$ we have

$$
\left\|u_{\partial \mathfrak{M}}\right\|_{L^{2}(\Gamma)} \leq C_{8}\left(\left|u_{\mathcal{T}}\right|_{1, \mathcal{T}}+\left\|u_{\mathfrak{M}}\right\|_{L^{2}(\Omega)}\right) .
$$

We can also remark that for a quasi-uniform mesh family $\left(\mathcal{T}^{(i)}\right)_{i \in \mathbb{N}}$ (see Definition 2.4) and for any $q \geq 1$, there exists a uniform constant $C_{9}>0$ (depending on $q$ and $\operatorname{reg}_{\text {unif }}(\mathcal{T})$ ) such that

$$
\sup _{\mathcal{K} \in \mathfrak{M}}\left|u_{\mathcal{K}}\right| \leq \frac{C_{9}}{\operatorname{size}\left(\mathcal{T}^{(i)}\right)^{2 / q}}\left\|u_{\mathfrak{M}}\right\|_{L^{q}(\Omega)}, \quad \forall u_{\mathfrak{M}} \in \mathbb{R}^{\mathfrak{M}}
$$

3. The finite-volume scheme . This section is devoted to the presentation of the numerical scheme. We refer the reader to [22] for the proofs of the energy stability, the existence of a discrete solution and the convergence analysis.

For the time discretization, let $N \in \mathbb{N}$ and $\Delta t=\frac{T}{N}$ be the time step. For any $n \in$ $\{0, \cdots, N\}$ we define $t^{n}=n \Delta t$. Then, at time $t^{n}$, the unknowns are denoted by

$$
c_{\mathcal{T}}^{n}=\left(\begin{array}{c}
\left(c_{\mathcal{K}}^{n}\right)_{\mathcal{K} \in \mathfrak{M}} \\
\left(c_{\mathcal{L}}^{n}\right)_{\mathcal{L} \in \partial \mathfrak{M}}
\end{array}\right) \text { and } \mu_{\mathcal{T}}^{n}=\left(\begin{array}{c}
\left(\mu_{\mathcal{K}}^{n}\right)_{\mathcal{K} \in \mathfrak{M}} \\
\left(\mu_{\mathcal{L}}^{n}\right)_{\mathcal{L} \in \partial \mathfrak{M}}
\end{array}\right) \text {. }
$$

Since $\mu$ is associated with the homogeneous Neumann boundary condition, for any $\mathcal{L} \in \partial \mathfrak{M}$ we have $\mu_{\mathcal{L}}^{n}=\mu_{\mathcal{K}}^{n}$ where $\mathcal{K} \in \mathfrak{M}$ is the interior control volume such that $\mathcal{L} \subset \partial \mathcal{K}$.

To obtain the finite-volume approximation of problem (1.1) we integrate the continuous equations (1.1a) and (1.1b) on all interior control volumes $\mathcal{K} \in \mathfrak{M}$ and we use a consistent twopoint flux approximation for the Laplace operators (associated with the Neumann boundary condition for $\mu$ ). Then we integrate dynamic boundary condition (1.1c) on all boundary control volumes $\mathcal{L} \in \partial \mathfrak{M}$ and we use a consistent two-point flux approximation for the Laplace-Beltrami operator.

As regards the discretization of nonlinear terms $f_{b}^{\prime}$ and $f_{s}^{\prime}$ (denoted by $d^{f_{b}}$ and $d^{f_{s}}$ respectively) we use two different discretizations (see Definition 3.1): the classical implicit discretization and a semi-implicit discretization which enables us to obtain an energy estimate unconditionally stable.

The problem is then written as follows. For a given $c_{\mathcal{T}}^{n} \in \mathbb{R}^{\mathcal{T}}$, find $\left(c_{\mathcal{T}}^{n+1}, \mu_{\mathcal{T}}^{n+1}\right) \in \mathbb{R}^{\mathcal{T}} \times \mathbb{R}^{\mathcal{T}}$ such that for any $\mathcal{K} \in \mathfrak{M}, \mathcal{L} \in \partial \mathfrak{M}$,

$$
m_{\underline{\mathcal{K}}} \frac{c_{\mathcal{K}}^{n+1}-c_{\mathcal{K}}^{n}}{\Delta t}=-\sum_{\sigma \in \mathcal{E}_{\mathcal{K}}^{i n t}} \frac{m_{\sigma}}{d_{\mathcal{K}, \mathcal{L}}}\left(\mu_{\mathcal{K}}^{n+1}-\mu_{\mathcal{L}}^{n+1}\right)
$$




$$
\begin{aligned}
m_{\underline{\mathcal{K}}} \mu_{\mathcal{K}}^{n+1}= & \sum_{\sigma \in \mathcal{E}_{\mathcal{K}}^{i n t}} \frac{m_{\sigma}}{d_{\mathcal{K}, \mathcal{L}}}\left(c_{\mathcal{K}}^{n+1}-c_{\mathcal{L}}^{n+1}\right)+\sum_{\sigma \in \mathcal{E}_{\mathcal{K}}^{e x t}} \frac{m_{\mathbf{e}_{\mathcal{L}}}}{d_{\mathcal{K}, \mathcal{L}}}\left(c_{\mathcal{K}}^{n+1}-c_{\mathcal{L}}^{n+1}\right) \\
& +m_{\underline{\mathcal{K}}} d^{f_{b}}\left(c_{\mathcal{K}}^{n}, c_{\mathcal{K}}^{n+1}\right) ; \\
m_{\mathbf{e}_{\mathcal{L}}} \frac{c_{\mathcal{L}}^{n+1}-c_{\mathcal{L}}^{n}}{\Delta t}= & -\sum_{\mathbf{v} \in \mathcal{V}_{\mathcal{L}}} \frac{\left(c_{\mathcal{L}}^{n+1}-c_{\mathcal{L}^{\prime}}^{n+1}\right)}{d_{\mathcal{L}, \mathcal{L}^{\prime}}}-m_{\mathbf{e}_{\mathcal{L}}} d^{f_{s}}\left(c_{\mathcal{L}}^{n}, c_{\mathcal{L}}^{n+1}\right)-\frac{m_{\mathbf{e}_{\mathcal{L}}}}{d_{\mathcal{K}, \mathcal{L}}}\left(c_{\mathcal{L}}^{n+1}-c_{\mathcal{K}}^{n+1}\right) .
\end{aligned}
$$

Definition 3.1 (Discretization of nonlinear terms). The implicit discretization is defined as follows: for any $\mathcal{K} \in \mathfrak{M}, \mathcal{L} \in \partial \mathfrak{M}$,

$$
d^{f_{b}}\left(c_{\mathcal{K}}^{n}, c_{\mathcal{K}}^{n+1}\right)=f_{b}^{\prime}\left(c_{\mathcal{K}}^{n+1}\right) \text { and } d^{f_{s}}\left(c_{\mathcal{L}}^{n}, c_{\mathcal{L}}^{n+1}\right)=f_{s}^{\prime}\left(c_{\mathcal{L}}^{n+1}\right) .
$$

As regards the semi-implicit discretization, for $* \in\{b, s\}$ we note

$$
d^{f_{*}}(x, y)=\frac{f_{*}(x)-f_{*}(y)}{x-y}, \forall x, y, x \neq y \quad \text { and } \quad d^{f_{*}}(x, x)=f_{*}^{\prime}(x), \forall x .
$$

We remark that in practice we use a polynomial function for the potential $f_{*}$ and that $d^{f_{*}}(x, y)$ is a polynomial function in the variables $x, y$. Thus, from a computational point of view, we do not have numerical instability when $x$ is too close to $y$.

Proposition 3.2. Using the semi-implicit discretization, the discrete energy is dissipated as follows: if $c_{\mathcal{T}}^{n}$ is given and $\left(c_{\mathcal{T}}^{n+1}, \mu_{\mathcal{T}}^{n+1}\right)$ is solution to problem (3.1), then there exists $C_{10}>0$ independent of $\Delta t$ and $\mathcal{T}$ such that for any $\Delta t$

$$
\begin{aligned}
\mathcal{F}_{\mathcal{T}}\left(c_{\mathcal{T}}^{n+1}\right)-\mathcal{F}_{\mathcal{T}}\left(c_{\mathcal{T}}^{n}\right)+C_{10}( & \Delta t\left|\mu_{\mathcal{T}}^{n+1}\right|_{1, \mathcal{T}}^{2}+\frac{1}{\Delta t} \| c_{\partial \mathfrak{M}}^{n+1}-\left.c_{\partial \mathfrak{M}}^{n}\right|_{0, \partial \mathfrak{M}} ^{2} \\
& \left.+\frac{1}{2}\left|c_{\mathcal{T}}^{n+1}-c_{\mathcal{T}}^{n}\right|_{1, \mathcal{T}}^{2}+\frac{1}{2}\left|c_{\partial \mathfrak{M}}^{n+1}-c_{\partial \mathfrak{M}}^{n}\right|_{1, \partial \mathfrak{M}}^{2}\right) \leq 0 .
\end{aligned}
$$

If we use an implicit discretization of the nonlinear terms, we also obtain (3.2) but with a condition $\Delta t \leq \Delta t_{0}$ (with $\Delta t_{0}$ depending on the parameters of the equation).

We note that summing equation (3.1a) for all $\mathcal{K} \in \mathfrak{M}$ we have the volume conservation at the discrete level

$$
|\underline{\Omega}| m_{\underline{\Omega}}\left(c_{\mathfrak{M}}^{n}\right):=\sum_{\mathcal{K} \in \mathfrak{M}} m_{\underline{\mathcal{K}}} c_{\mathcal{K}}^{n}=\sum_{\mathcal{K} \in \mathfrak{M}} m_{\underline{\mathcal{K}}} c_{\mathcal{K}}^{0}:=|\underline{\Omega}| m_{\underline{\Omega}}\left(c_{\mathfrak{M}}^{0}\right), \quad \forall n \in\{1, \ldots, N\} .
$$

4. Error estimate for the fully-discrete scheme. We can now enter in the heart of the matter. The most delicate point in the proof of the error estimate (Theorem 4.2) comes from the nonlinear term $f_{b}^{\prime}(c)$ for which we have to pay special attention. For this, we are inspired by methods described in $[9,19]$ for the Neumann boundary condition and a finiteelement approximation. However some supplementary difficulties arise in our case. First, the finite-volume framework complicates the study of this term. Indeed, when we use a conform finite-element method, we work (for the space discretization at least) on $H^{1}$-conformal spaces. That is not the case in the framework of finite-volume method where we resort to discrete spaces. Moreover, we use here two different discretizations for the nonlinear term $f_{b}^{\prime}$ (see Definition 3.1). The second one, that is the semi-implicit discretization, is more difficult to study, which complicates again the proof of Theorem 4.2.

4.1. Main result. In this section we present the error estimate (Theorem 4.2) between the center-value projection (see Definition 4.1) of the exact solution and the discrete solution obtained by solving problem (3.1). 
Definition 4.1 (Center-value projection). The center-value projection $\mathbb{P}_{\mathcal{T}}^{c}: \mathcal{C}^{0}(\bar{\Omega}) \rightarrow \mathbb{R}^{\mathcal{T}}$ is defined as follows. For any $u \in \mathcal{C}^{0}(\bar{\Omega})$, we set $\mathbb{P}_{\mathcal{T}}^{c} u=\left(\mathbb{P}_{\mathfrak{M}}^{c} u, \mathbb{P}_{\partial \mathfrak{M}}^{c} u\right)$ with

$$
\mathbb{P}_{\mathfrak{M}}^{c} u=\left(\mathbb{P}_{\mathcal{K}}^{c} u\right)_{\mathcal{K} \in \mathfrak{M}}=\left(u\left(x_{\mathcal{K}}\right)\right)_{\mathcal{K} \in \mathfrak{M}} \text { and } \mathbb{P}_{\partial \mathfrak{M}}^{c} u=\left(\mathbb{P}_{\mathcal{L}}^{c} u\right)_{\mathcal{L} \in \partial \mathfrak{M}}=\left(u_{\mid \Gamma}\left(x_{\mathcal{L}}\right)\right)_{\mathcal{L} \in \partial \mathfrak{M}} .
$$

TheOREM 4.2 (Error estimate). Let $(c, \mu) \in \mathcal{C}^{3}([0, T] \times \bar{\Omega}) \times \mathcal{C}^{2}([0, T] \times \bar{\Omega})$ be a solution to the Cahn-Hilliard equation (1.1) associated with the initial data $c^{0} \in \mathcal{C}^{2}(\bar{\Omega})$. Let $M>0$ be such that $\|c\|_{L^{\infty}\left(0, T ; L^{\infty}(\Omega)\right)} \leq M$ and $M^{\prime}>M$. Then setting $c_{\mathcal{T}}^{0}=\mathbb{P}_{\mathcal{T}}^{c} c^{0}$, for any solution $\left(c_{\mathcal{T}}^{n}, \mu_{\mathcal{T}}^{n}\right)$ to problem (3.1) satisfying

$$
\sup \left(\sup _{\mathcal{K} \in \mathfrak{M}}\left|c_{\mathcal{K}}^{n}\right|, \sup _{\mathcal{L} \in \partial \mathfrak{M}}\left|c_{\mathcal{L}}^{n}\right|\right) \leq M^{\prime}, \quad \forall n \in \llbracket 0, N \rrbracket,
$$

there exists $C_{11}>0$ (depending on $M$ and $M^{\prime}$ ) such that the following estimate holds (with $\Delta t$ small enough),

$$
\sup _{n \in \llbracket 0, N \rrbracket}\left\|\mathbb{P}_{\mathcal{T}}^{c} c\left(t^{n}\right)-c_{\mathcal{T}}^{n}\right\|_{1, \mathcal{T}}+\sup _{n \in \llbracket 0, N \rrbracket}\left\|\mathbb{P}_{\partial \mathfrak{M}}^{c} c\left(t^{n}\right)-c_{\partial \mathfrak{M}}^{n}\right\|_{1, \partial \mathfrak{M}} \leq C_{11}(\Delta t+\operatorname{size}(\mathcal{T})) .
$$

Note that the proof given in Section 4.3 does not use assumption (4.1). Thus, in this case, to perform the proof we need some Lipschitz type regularity properties on the potentials and so we work with truncated Lipschitz potentials instead of initial potentials. However note that,

- If we observe (numerically for instance) that bound (4.1) is satisfied, then Theorem 4.2 holds with the initials potentials.

- Otherwise, Corollary 4.3 proves that we can carry out the proof of Theorem 4.2 with the truncated functions (which satisfy all the necessary regularity assumptions) and then, for a quasi-uniform mesh family, we obtain Theorem 4.2 with the initial potentials.

Corollary 4.3. For a quasi-uniform mesh family, if $\Delta t$ and $\operatorname{size}(\mathcal{T})$ are so that $\Delta t \leq$ $\operatorname{size}(\mathcal{T})^{\alpha}$ (for an arbitrary value $\alpha>0$ ), then assumption (4.1) is still satisfied for $\Delta t$ and $\operatorname{size}(\mathcal{T})$ small enough. Therefore there exists at least one solution $c_{\mathcal{T}}^{n}$ to discrete problem (3.1) which satisfies (4.1) and Theorem 4.2 holds for this solution.

Note that there may exist solutions of problem (3.1) for which (4.1) does not hold.

Proof. The main steps are the following:

1. We introduce truncated Lipschitz continuous functions in the following way. The exact solution $c$ of continuous problem (1.1) is supposed to belong to $\mathcal{C}^{3}([0, T] \times \bar{\Omega})$. Thus, there exists $M>0$ such that $\|c\|_{L^{\infty}\left(0, T ; L^{\infty}(\Omega)\right)} \leq M$. Let $M^{\prime}>M$, we choose truncated potentials $\widetilde{f}_{b}$ and $\widetilde{f}_{s}$ of the initial potentials $f_{b}$ and $f_{s}$ satisfying $\widetilde{f}_{b}=f_{b}$ and $\widetilde{f}_{s}=f_{s}$ on $\left[-M^{\prime}, M^{\prime}\right]$, and which are constant at the infinity. These truncated functions, and all their derivatives, are Lipschitz continuous. Moreover, the definition of the semi-implicit discretization of nonlinear terms implies

$$
d^{f_{b}}(x, y)=\int_{0}^{1} f_{b}^{\prime}(x+s(y-x)) \mathrm{d} s \text { and } d^{f_{s}}(x, y)=\int_{0}^{1} f_{s}^{\prime}(x+s(y-x)) \mathrm{d} s .
$$

Thus the semi-implicit discretization of $\widetilde{f}_{b}^{\prime}\left(\right.$ resp. $\left.\widetilde{f}_{s}^{\prime}\right)$ coincides with $d^{f_{b}}\left(\right.$ resp. $\left.d^{f_{s}}\right)$ on $\left[-M^{\prime}, M^{\prime}\right]^{2}$ and is Lipschitz continuous.

2. We can derive the proof of Theorem 4.2 with the truncated potentials (see Section 4.3) instead of the initial potentials (and so with $c_{\mathcal{T}}^{n}$ a solution to discrete problem (3.1) but with the truncated potentials). We obtain estimate (4.2) for this problem with constant $C_{11}$ depending on $M^{\prime}$. 
3. For a mesh belonging to a quasi-uniform mesh family, gathering estimate (2.5), Lemma 2.8 and Theorem 4.2 we deduce

$$
\sup _{\mathcal{K} \in \mathfrak{M}}\left|\mathbb{P}_{\mathcal{K}}^{c} c\left(t^{n}\right)-c_{\mathcal{K}}^{n}\right| \leq C_{6} C_{11} C_{9}\left(\frac{\Delta t}{\operatorname{size}(\mathcal{T})^{2 / q}}+\operatorname{size}(\mathcal{T})^{1-2 / q}\right)
$$

Moreover, thanks to Lemma 2.9 and Theorem 4.2 we also have

$$
\sup _{\mathcal{L} \in \partial \mathfrak{M}}\left|\mathbb{P}_{\mathcal{L}}^{c} c\left(t^{n}\right)-c_{\mathcal{L}}^{n}\right| \leq C_{7} C_{11}(\Delta t+\operatorname{size}(\mathcal{T})) .
$$

Thus, if $\Delta t$ and $\operatorname{size}(\mathcal{T})$ tend to 0 with $\Delta t \leq C \operatorname{size}(\mathcal{T})^{\alpha}$ for some $\alpha>0$, we have that bound (4.1) is satisfied for any approximate solution. Moreover, since the functions $f_{b}$ (resp. $f_{s}$ ) and $\widetilde{f}_{b}$ (resp. $\widetilde{f}_{s}$ ) coincide on $\left[-M^{\prime}, M^{\prime}\right]$, if $c_{\mathcal{T}}^{n}$ is solution to discrete problem (3.1) with the truncated potentials, it also holds with the initial potentials (and reciprocally).

Remark 4.4. From a computational point of view, we can check that assumption (4.1) holds when we use the potentials $f_{b}$ and $f_{s}$.

In order to prove the error estimate theorem we have to use a priori bounds on the discrete solution (obtained thanks to the discrete energy estimate given in Proposition 3.2).

Proposition 4.5 (Bounds of the discrete solutions, [22, Proposition 4.4]). For any $c^{0} \in$ $\mathcal{C}^{2}(\bar{\Omega})$, let $c_{\mathcal{T}}^{0}=\mathbb{P}_{\mathcal{T}}^{c} c^{0}$ and $\left(c_{\mathcal{T}}^{n}, \mu_{\mathcal{T}}^{n}\right) \in \mathbb{R}^{\mathcal{T}} \times \mathbb{R}^{\mathcal{T}}$ be a solution to problem (3.1). Then, there exist positive constants $M_{1}, M_{2}, M_{3}, M_{4}$ and $M_{5}$ independent of $\Delta t$ and $\operatorname{size}(\mathcal{T})$ such that

$$
\begin{gathered}
\sup _{n \leq N}\left\|c_{\mathcal{T}}^{n}\right\|_{1, \mathcal{T}} \leq M_{1}, \quad \sup _{n \leq N}\left\|c_{\partial \mathfrak{M}}^{n}\right\|_{1, \partial \mathfrak{M}} \leq M_{2}, \\
\sum_{n=0}^{N-1} \Delta t\left\|\mu_{\mathcal{T}}^{n+1}\right\|_{1, \mathcal{T}}^{2} \leq M_{3}, \quad \sum_{n=0}^{N-1} \Delta t\left\|\frac{c_{\mathcal{T}}^{n+1}-c_{\mathcal{T}}^{n}}{\Delta t}\right\|_{1, \mathcal{T}}^{2} \leq \frac{M_{4}}{\Delta t}, \\
\text { and } \quad \sum_{n=0}^{N-1} \Delta t\left\|\frac{c_{\partial \mathfrak{M}}^{n+1}-c_{\partial \mathfrak{M}}^{n}}{\Delta t}\right\|_{0, \partial \mathfrak{M}}^{2}+\Delta t^{2} \sum_{n=0}^{N-1}\left|\frac{c_{\partial \mathfrak{M}}^{n+1}-c_{\partial \mathfrak{M}}^{n}}{\Delta t}\right|_{1, \partial \mathfrak{M}}^{2} \leq M_{5} .
\end{gathered}
$$

We can remark that in [22] the proposition is proved by choosing the mean-value projection on all control volumes as discrete initial data for the initial concentration $c^{0}$. In fact, when the initial data is not enough regular, we have to choose this projection to obtain the $a$ priori bounds. However, when the initial data belongs to $\mathcal{C}^{2}(\bar{\Omega})$ the center-value projection is sufficient. Indeed, thanks to the mean-value theorem, we obtain a bound on the $H^{1}$-seminorm of $c_{\mathcal{T}}^{0}$ which allows us to prove the proposition as in [22].

4.2. Discrete projections. To prove Theorem 4.2 we have to define another projection: the elliptic projection. These projection is in fact the solution of a suitable Laplace problem and thus depends on the boundary condition that we want to impose. Therefore, the definition of the elliptic projection for the chemical potential (see Definition 4.6) and for the order parameter (see Definition 4.7) are different.

DeFinition 4.6 (Elliptic projection with Neumann boundary conditions).

We define the space $H_{N}^{2}(\Omega)=\left\{u \in H^{2}(\Omega): \nabla u \cdot \overrightarrow{\mathbf{n}}=0\right.$ on $\left.\Gamma\right\}$, then the elliptic projection $\mathbb{P}_{\mathcal{T}}^{\text {ell,N }}: H_{N}^{2}(\Omega) \rightarrow \mathbb{R}^{\mathcal{T}}$ is defined as follows. For any $u \in H_{N}^{2}(\Omega), \mathbb{P}_{\mathcal{T}}^{\text {ell, } \mathrm{N}} u$ is the solution to the following discrete Laplace problem. 


$$
\begin{aligned}
& \text { Find } v_{\mathcal{T}} \in \mathbb{R}^{\mathcal{T}} \text { such that } \sum_{\mathcal{K} \in \mathfrak{M}} m_{\underline{\mathcal{K}}} v_{\mathcal{K}}=\int_{\Omega} u \text { and } \\
& \qquad \sum_{\sigma \in \mathcal{E}_{\mathcal{K}}^{i n t}} m_{\sigma} \frac{v_{\mathcal{K}}-v_{\mathcal{L}}}{d_{\mathcal{K}, \mathcal{L}}}=-\int_{\mathcal{K}} \Delta u(x) \mathrm{d} x, \quad \forall \mathcal{K} \in \mathfrak{M} .
\end{aligned}
$$

DeFInITION 4.7 (Elliptic projection with Ventcell boundary conditions).

The elliptic projection $\mathbb{P}_{\mathcal{T}}^{\text {ell, } \mathrm{D}}: H_{\Gamma}^{2}(\Omega) \rightarrow \mathbb{R}^{\mathcal{T}}$ is defined as follows. For any $u \in H_{\Gamma}^{2}(\Omega)$, $\mathbb{P}_{\mathcal{T}}^{\text {ell,D }} u$ is the solution to the following discrete Laplace problem.

Find $v_{\mathcal{T}} \in \mathbb{R}^{\mathcal{T}}$ such that $\sum_{\mathcal{K} \in \mathfrak{M}} m_{\underline{\mathcal{K}}} v_{\mathcal{K}}=\int_{\Omega} u$ and for any $\mathcal{K} \in \mathfrak{M}, \mathcal{L} \in \partial \mathfrak{M}$,

$$
\left\{\begin{array}{l}
\sum_{\sigma \in \mathcal{E}_{\mathcal{K}}^{\text {int }}} m_{\sigma} \frac{v_{\mathcal{K}}-v_{\mathcal{L}}}{d_{\mathcal{K}, \mathcal{L}}}+\sum_{\sigma \in \mathcal{E}_{\mathcal{K}}^{\text {ext }}} m_{\mathbf{e}_{\mathcal{L}}} \frac{v_{\mathcal{K}}-v_{\mathcal{L}}}{d_{\mathcal{K}, \mathcal{L}}}=-\int_{\mathcal{K}} \Delta u(x) \mathrm{d} x \\
\sum_{\mathbf{v} \in \mathcal{V}_{\mathcal{L}}} \frac{v_{\mathcal{L}}-v_{\mathcal{L}^{\prime}}}{d_{\mathcal{L}, \mathcal{L}^{\prime}}}+m_{\mathbf{e}_{\mathcal{L}}} \frac{v_{\mathcal{L}}-v_{\mathcal{K}}}{d_{\mathcal{K}, \mathcal{L}}}=-\int_{\mathcal{L}} \Delta_{\Gamma} u_{I \Gamma}(x) \mathrm{d} \sigma(x)+\int_{\mathcal{L}} \nabla u(x) \cdot \overrightarrow{\mathbf{n}}(x) \mathrm{d} \sigma(x) .
\end{array}\right.
$$

Remark 4.8. The elliptic projection is the solution to the finite-volume two-point flux approximation of the continuous problem

$$
\left\{\begin{aligned}
-\Delta v & =f & & \text { in } \Omega, \\
-\alpha \Delta_{\Gamma} v+\partial_{n} v & =g & & \text { on } \Gamma,
\end{aligned}\right.
$$

with $f=-\Delta u$ and

- $\alpha=0, g=0$ for the elliptic projection $\mathbb{P}_{\mathcal{T}}^{\text {ell, }}$;

- $\alpha=1, g=-\Delta_{\Gamma} u+\partial_{n} u$ for the elliptic projection $\mathbb{P}_{\mathcal{T}}^{\text {ell,D }}$.

When $u$ is a time-dependent function, for a fixed time $t \in \mathbb{R}$, we denote $\mathbb{P}_{\mathcal{T}}^{\text {ell, }}(u(t))$ and $\mathbb{P}_{\mathcal{T}}^{\text {ell,D }}(u(t))$ the elliptic projections of the function $v=u(t,$.$) .$

In order to prove Theorem 4.2 we have to relate the different discrete projections to the solution of the continuous problem (1.1). With this in mind we give below several properties which will be used all along the proof in Section 4.3.

Thanks to the Taylor's formulas and the Jensen inequality we can easily prove the following estimates between an arbitrary function and its center-value projection.

Lemma 4.9. Let $u \in H^{2}(\Omega)$, there exists $C_{12}>0$ independent of $\operatorname{size}(\mathcal{T})$ such that

$$
\left\|u-\mathbb{P}_{\mathfrak{M}}^{c} u\right\|_{L^{2}(\Omega)} \leq C_{12} \operatorname{size}(\mathcal{T})\|\nabla u\|_{H^{1}(\Omega)} .
$$

Moreover, there exists $C_{13}>0$ independent of $\operatorname{size}(\mathcal{T})$ such that for any $u_{1 \Gamma} \in H^{1}(\Gamma)$,

$$
\left\|u_{\mid \Gamma}-\mathbb{P}_{\partial \mathfrak{M}}^{c} u_{\mid \Gamma}\right\|_{L^{2}(\Gamma)} \leq C_{13} \operatorname{size}(\mathcal{T})\left\|\nabla_{\Gamma} u_{\mid \Gamma}\right\|_{L^{2}(\Gamma)} .
$$

Since the introduction of the elliptic projection is essential to prove the error estimate theorem, we also need to control this projection.

Proposition 4.10. Let $u \in H_{N}^{2}(\Omega)$ be a Lipschitz continuous function, there exists $C_{14}>$ 0 depending only on $\Omega$ such that

$$
\left\|\mathbb{P}_{\mathfrak{M}}^{\mathrm{ell}, \mathrm{N}} u-u\right\|_{L^{2}(\Omega)}+\left|\mathbb{P}_{\mathcal{T}}^{\mathrm{ell}, \mathrm{N}} u-\mathbb{P}_{\mathcal{T}}^{c} u\right|_{1, \mathcal{T}} \leq C_{14} \operatorname{size}(\mathcal{T})\|u\|_{H^{2}(\Omega)} .
$$


Proof. Thanks to Definition 4.6 of the elliptic projection, $\mathbb{P}_{\mathcal{T}}^{\mathrm{ell}, \mathrm{N}} u$ is solution to discrete problem (4.3). Thus the difference $\mathbb{P}_{\mathcal{T}}^{\text {ell, }}{ }^{-} u-\mathbb{P}_{\mathcal{T}}^{c} u$ is the error associated to problem (4.5) with $\alpha=g=0$. Thanks to the error estimate for the Laplace problem (4.5) for the two-point flux approximation scheme (see for example [11, Section 10.3]) we obtain the expected estimates.D

As regards the analogous proposition in the case of Ventcell boundary condition, the proof does not seem available in the literature and so we propose a complete proof in the Appendix.

Proposition 4.11. Let $u \in H_{\Gamma}^{2}(\Omega)$ be a Lipschitz continuous function, there exists $C_{15}>0$ depending only on $\Omega$ and $\operatorname{reg}(\mathcal{T})$ such that

$$
\left\|\mathbb{P}_{\mathcal{T}}^{\mathrm{ell}, \mathrm{D}} u-u\right\|_{L^{2}(\Omega)}+\left\|\mathbb{P}_{\partial \mathfrak{M}}^{\mathrm{ell}, \mathrm{D}} u-u\right\|_{L^{2}(\Gamma)} \leq C_{15} \operatorname{size}(\mathcal{T})\|u\|_{H_{\Gamma}^{2}(\Omega)}
$$

and

$$
\left|\mathbb{P}_{\mathcal{T}}^{\mathrm{ell}, \mathrm{D}} u-\mathbb{P}_{\mathcal{T}}^{c} u\right|_{1, \mathcal{T}}+\left|\mathbb{P}_{\partial \mathfrak{M}}^{\mathrm{ell}, \mathrm{D}} u-\mathbb{P}_{\partial \mathfrak{M}}^{c} u\right|_{1, \partial \mathfrak{M}} \leq C_{15} \operatorname{size}(\mathcal{T})\|u\|_{H_{\Gamma}^{2}(\Omega)}
$$

4.3. Proof of Theorem 4.2. This section is devoted to the proof of Theorem 4.2. We decompose the proof in three steps. First, we use the scheme to decompose the different components of the error. Then, we control all the terms and prove that they tend to 0 when the mesh size and the time step tend to 0. Finally we use the discrete Gronwall lemma to conclude the proof.

In the proof different components of the total error appear. Thus we decompose the error as follows.

DEFINITION 4.12 (Error). Let $u:(0, T) \times \Omega \rightarrow \mathbb{R}$ and $u_{\mathcal{T}}^{n}$ be a finite-volume approximation of $u$ at time $t^{n}$. We denote by $e_{\mathcal{T}}^{u, n} \in \mathbb{R}^{\mathcal{T}}$ the error associated with $u$ at time $t=t^{n}$ defined as follows

$$
e_{\mathcal{T}}^{u, n}=\ddot{e}_{\mathcal{T}}^{u, n}+\dot{e}_{\mathcal{T}}^{u, n} \text { with } \ddot{e}_{\mathcal{T}}^{u, n}=\mathbb{P}_{\mathcal{T}}^{c} u\left(t^{n}\right)-\mathbb{P}_{\mathcal{T}}^{\mathrm{ell}, *} u\left(t^{n}\right) \text { and } \dot{e}_{\mathcal{T}}^{u, n}=\mathbb{P}_{\mathcal{T}}^{\mathrm{ell}, *} u\left(t^{n}\right)-u_{\mathcal{T}}^{n}
$$

with $*=\{\mathrm{N}, \mathrm{D}\}$ depending on the boundary condition associated with $u$.

We also define $\bar{e}_{\mathcal{T}}^{u, n}=u\left(t^{n}, \cdot\right)-\frac{m_{\mathcal{T}}}{m_{\mathcal{T}}} \mathbb{P}_{\mathcal{T}}^{\mathrm{ell}, *} u\left(t^{n}\right)$.

4.3.1. Different contributions of the error. First, subtracting the scheme and the continuous problem we identify the different components of the error (Proposition 4.13). Then, we separate the error into two parts: the error $\dot{e}_{\mathcal{T}}$ between the elliptic projection of the exact solution and the approximate solution in the left-hand side, and all the other contributions in the right-hand side (Proposition 4.14).

Proposition 4.13. Let us consider a couple $(c, \mu)$ solution to the continuous Cahn-Hilliard equation (1.1) and a couple $\left(c_{\mathcal{T}}^{n+1}, \mu_{\mathcal{T}}^{n+1}\right)$ solution to the finite-volume scheme (3.1). Then, the 
following equality holds

$$
\begin{aligned}
\Delta & t\left|\dot{e}_{\mathcal{T}}^{\mu, n+1}\right|_{1, \mathcal{T}}^{2}+\frac{1}{2}\left(\left|\dot{e}_{\mathcal{T}}^{c, n+1}\right|_{1, \mathcal{T}}^{2}-\left|\dot{e}_{\mathcal{T}}^{c, n}\right|_{1, \mathcal{T}}^{2}+\left|\dot{e}_{\mathcal{T}}^{c, n+1}-\dot{e}_{\mathcal{T}}^{c, n}\right|_{1, \mathcal{T}}^{2}\right) \\
& +\frac{1}{\Delta t} \| \dot{e}_{\partial \mathfrak{M}}^{c, n+1}-\left.\dot{e}_{\partial \mathfrak{M}}^{c, n}\right|_{0, \partial \mathfrak{M}} ^{2}+\frac{1}{2}\left(\left|\dot{e}_{\partial \mathfrak{M}}^{c, n+1}\right|_{1, \partial \mathfrak{M}}^{2}-\left|\dot{e}_{\partial \mathfrak{M}}^{c, n}\right|_{1, \partial \mathfrak{M}}^{2}+\left|\dot{e}_{\partial \mathfrak{M}}^{c, n+1}-\dot{e}_{\partial \mathfrak{M}}^{c, n}\right|_{1, \partial \mathfrak{M}}^{2}\right) \\
= & \Delta t\left(R_{c}^{n+1}, \dot{e}_{\mathfrak{M}}^{\mu, n+1}\right)_{L^{2}(\Omega)}-\left(\bar{e}_{\mathfrak{M}}^{c, n+1}-\bar{e}_{\mathfrak{M}}^{c, n}, \dot{e}_{\mathfrak{M}}^{\mu, n+1}\right)_{L^{2}(\Omega)}+\left(\bar{e}_{\mathfrak{M}}^{\mu, n+1}, \dot{e}_{\mathfrak{M}}^{c, n+1}-\dot{e}_{\mathfrak{M}}^{c, n}\right)_{L^{2}(\Omega)} \\
& +\left(R_{c_{1 \Gamma}}^{n+1}, \dot{e}_{\partial \mathfrak{M}}^{c, n+1}-\dot{e}_{\partial \mathfrak{M}}^{c, n}\right)_{L^{2}(\Gamma)}-\left(\frac{\bar{e}_{\partial \mathfrak{M}}^{c, n+1}-\bar{e}_{\partial \mathfrak{M}}^{c, n}}{\Delta t}, \dot{e}_{\partial \mathfrak{M}}^{c, n+1}-\dot{e}_{\partial \mathfrak{M}}^{c, n}\right)_{L^{2}(\Gamma)} \\
& -\left(f_{b}^{\prime}\left(c\left(t^{n+1}, \cdot\right)\right)-\frac{m_{\mathfrak{M}}}{m_{\mathfrak{M}}} d^{f_{b}}\left(c_{\mathfrak{M}}^{n}, c_{\mathfrak{M}}^{n+1}\right), \dot{e}_{\mathfrak{M}}^{c, n+1}-\dot{e}_{\mathfrak{M}}^{c, n}\right)_{L^{2}(\Omega)} \\
& -\left(f_{s}^{\prime}\left(c_{1 \Gamma}\left(t^{n+1}, \cdot\right)\right)-\frac{m_{\partial \mathfrak{M}}}{m_{\partial \mathfrak{M}}} d^{f_{s}}\left(c_{\partial \mathfrak{M}}^{n}, c_{\partial \mathfrak{M}}^{n+1}\right), \dot{e}_{\partial \mathfrak{M}}^{c, n+1}-\dot{e}_{\partial \mathfrak{M}}^{c, n}\right)_{L^{2}(\Gamma)}
\end{aligned}
$$

where the terms $R_{c}^{n+1}$ and $R_{c_{\Gamma} \Gamma}^{n+1}$ are defined as follows

$$
R_{c_{(เ \Gamma)}}^{n+1}=\frac{c_{(เ \Gamma)}\left(t^{n+1}, x\right)-c_{(เ \Gamma)}\left(t^{n}, x\right)}{\Delta t}-\partial_{t} c_{((\Gamma)}\left(t^{n+1}, x\right) .
$$

Proof. Let $(c, \mu)$ be a solution to the Cahn-Hilliard equation (1.1). Applying Definition 4.6 of the elliptic projection with Neumann boundary condition to $\mu$, for any $\mathcal{K} \in \mathfrak{M}$ we have

$$
\int_{\mathcal{K}} \partial_{t} c\left(t^{n+1}, x\right) \mathrm{d} x+\sum_{\sigma \in \mathcal{E}_{\mathcal{K}}^{\text {int }}} m_{\sigma} \frac{\mathbb{P}_{\mathcal{K}}^{\mathrm{ell}, \mathrm{N}} \mu\left(t^{n+1}\right)-\mathbb{P}_{\mathcal{L}}^{\mathrm{ell}, \mathrm{N}} \mu\left(t^{n+1}\right)}{d_{\mathcal{K}, \mathcal{L}}}=0 .
$$

In the same way, applying Definition 4.7 of the elliptic projection with Ventcell boundary condition to $c$, for any $\mathcal{K} \in \mathfrak{M}$ we have

$$
\begin{aligned}
\sum_{\sigma \in \mathcal{E}_{\mathcal{K}}^{i n t}} m_{\sigma} \frac{\mathbb{P}_{\mathcal{K}}^{\mathrm{ell}, \mathrm{D}} c\left(t^{n+1}\right)-\mathbb{P}_{\mathcal{L}}^{\mathrm{ell}, \mathrm{D}} c\left(t^{n+1}\right)}{d_{\mathcal{K}, \mathcal{L}}} & +\sum_{\sigma \in \mathcal{E}_{\mathcal{K}}^{\text {ext }}} m_{\mathbf{e}_{\mathcal{L}}} \frac{\mathbb{P}_{\mathcal{K}}^{\mathrm{ell}, \mathrm{D}} c\left(t^{n+1}\right)-\mathbb{P}_{\mathcal{L}}^{\mathrm{ell}, \mathrm{D}} c\left(t^{n+1}\right)}{d_{\mathcal{K}, \mathcal{L}}} \\
& +\int_{\mathcal{K}} f_{b}^{\prime}\left(c\left(t^{n+1}, x\right)\right) \mathrm{d} x-\int_{\mathcal{K}} \mu\left(t^{n+1}, x\right) \mathrm{d} x=0
\end{aligned}
$$

and for any $\mathcal{L} \in \partial \mathfrak{M}$,

$$
\begin{aligned}
& \sum_{\mathbf{v} \in \mathcal{V}_{\mathcal{L}}} \frac{\mathbb{P}_{\mathcal{L}}^{\mathrm{ell}, \mathrm{D}} c\left(t^{n+1}\right)-\mathbb{P}_{\mathcal{L}^{\prime}}^{\mathrm{ell}, \mathrm{D}} c\left(t^{n+1}\right)}{d_{\mathcal{L}, \mathcal{L}^{\prime}}}+m_{\mathbf{e}_{\mathcal{L}}} \frac{\mathbb{P}_{\mathcal{L}}^{\mathrm{ell}, \mathrm{D}} c\left(t^{n+1}\right)-\mathbb{P}_{\mathcal{K}}^{\mathrm{ell}, \mathrm{D}} c\left(t^{n+1}\right)}{d_{\mathcal{K}, \mathcal{L}}} \\
&+\int_{\mathcal{L}} f_{s}^{\prime}\left(c_{\mid \Gamma}\left(t^{n+1}, x\right)\right) \mathrm{d} \sigma(x)+\int_{\mathcal{L}} \partial_{t} c_{\mid \Gamma}\left(t^{n+1}, x\right) \mathrm{d} \sigma(x)=0
\end{aligned}
$$

where $\mathcal{K} \in \mathfrak{M}$ is the interior control volume such that $\mathcal{L} \in \partial \mathcal{K}$. Subtracting equation (3.1a) of discrete problem and equation (4.8) and using definition (4.7) of $R_{c}^{n+1}$ imply

$$
\begin{aligned}
\sum_{\sigma \in \mathcal{E}_{\mathcal{K}}^{i n t}} m_{\sigma} \frac{\dot{e}_{\mathcal{K}}^{\mu, n+1}-\dot{e}_{\mathcal{L}}^{\mu, n+1}}{d_{\mathcal{K}, \mathcal{L}}}+m_{\underline{\mathcal{K}}} \frac{\dot{e}_{\mathcal{K}}^{c, n+1}-\dot{e}_{\mathcal{K}}^{c, n}}{\Delta t}=\int_{\mathcal{K}} R_{c}^{n+1}(x) \mathrm{d} x \\
\quad-\int_{\mathcal{K}} \frac{\bar{e}_{\mathcal{K}}^{c, n+1}(x)-\bar{e}_{\mathcal{K}}^{c, n}(x)}{\Delta t} \mathrm{~d} x .
\end{aligned}
$$


Now, we subtract equation (3.1b) of the discrete problem and equation (4.9), then we obtain

$$
\begin{aligned}
\sum_{\sigma \in \mathcal{E}_{\mathcal{K}}^{i n t}} m_{\sigma} \frac{\dot{\mathcal{E}}_{\mathcal{K}}^{c, n+1}-\dot{e}_{\mathcal{L}}^{c, n+1}}{d_{\mathcal{K}, \mathcal{L}}} & +\sum_{\sigma \in \mathcal{E}_{\mathcal{K}}^{e x t}} m_{\mathbf{e}_{\mathcal{L}}} \frac{\dot{e}_{\mathcal{K}}^{c, n+1}-\dot{e}_{\mathcal{L}}^{c, n+1}}{d_{\mathcal{K}, \mathcal{L}}}-m_{\underline{\mathcal{K}}} \dot{e}_{\mathcal{K}}^{\mu, n+1} \\
= & \int_{\mathcal{K}} \bar{e}_{\mathcal{K}}^{\mu, n+1} \mathrm{~d} x-\int_{\mathcal{K}}\left(f_{b}^{\prime}\left(c\left(t^{n+1}, x\right)\right)-\frac{m_{\underline{\mathcal{K}}}}{m_{\mathcal{K}}} d^{f_{b}}\left(c_{\mathcal{K}}^{n}, c_{\mathcal{K}}^{n+1}\right)\right) \mathrm{d} x
\end{aligned}
$$

Subtracting equation (3.1c) of the discrete problem and equation (4.10) we have

$$
\begin{aligned}
& \sum_{\mathbf{v} \in \mathcal{V}_{\mathcal{L}}} \frac{\dot{e}_{\mathcal{L}}^{c, n+1}-\dot{e}_{\mathcal{L}^{\prime}}^{c, n+1}}{d_{\mathcal{L}, \mathcal{L}^{\prime}}}+m_{\mathbf{e}_{\mathcal{L}}} \frac{\dot{e}_{\mathcal{L}}^{c, n+1}-\dot{e}_{\mathcal{K}}^{c, n+1}}{d_{\mathcal{K}, \mathcal{L}}}+m_{\mathbf{e}_{\mathcal{L}}} \frac{\dot{e}_{\mathcal{L}}^{c, n+1}-\dot{e}_{\mathcal{L}}^{c, n}}{\Delta t}=\int_{\mathcal{L}} R_{c_{\mid \Gamma}}^{n+1}(x) \mathrm{d} \sigma(x) \\
& -\int_{\mathcal{L}} \frac{\bar{e}_{\mathcal{L}}^{c, n+1}(x)-\bar{e}_{\mathcal{L}}^{c, n}(x)}{\Delta t} \mathrm{~d} \sigma(x)-\int_{\mathcal{L}}\left(f_{s}^{\prime}\left(c_{\mid \Gamma}\left(t^{n+1}, x\right)\right)-\frac{m_{\mathbf{e}_{\mathcal{L}}}}{m_{\mathcal{L}}} d^{f_{s}}\left(c_{\mathcal{L}}^{n}, c_{\mathcal{L}}^{n+1}\right)\right) \mathrm{d} \sigma(x) .
\end{aligned}
$$

We multiply equation (4.11) by $v_{\mathcal{K}}$ and we sum up over all interior control volumes $\mathcal{K} \in \mathfrak{M}$, we obtain

$$
\llbracket \dot{e}_{\mathcal{T}}^{\mu, n+1}, v_{\mathcal{T}} \rrbracket_{1, \mathcal{T}}+\left(\frac{\dot{e}_{\mathfrak{M}}^{c, n+1}-\dot{e}_{\mathfrak{M}}^{c, n}}{\Delta t}, v_{\mathfrak{M}}\right)_{\mathfrak{M}}=\left(R_{c}^{n+1}, v_{\mathfrak{M}}\right)_{L^{2}(\Omega)}-\left(\frac{\bar{e}_{\mathfrak{M}}^{c, n+1}-\bar{e}_{\mathfrak{M}}^{c, n}}{\Delta t}, v_{\mathfrak{M}}\right)_{L^{2}(\Omega)}
$$

Now, we first multiply equation (4.12) by $u_{\mathcal{K}}$ and we sum up over all interior control volumes $\mathcal{K} \in \mathfrak{M}$. Then, we multiply equation (4.13) by $u_{\mathcal{L}}$ and we sum up over all boundary control volumes $\mathcal{L} \in \partial \mathfrak{M}$. Summing the resulting equalities, we have

$$
\begin{aligned}
\llbracket \dot{e}_{\mathcal{T}}^{c, n+1}, u_{\mathcal{T}} \rrbracket_{1, \mathcal{T}} & -\left(\dot{e}_{\mathfrak{M}}^{\mu, n+1}, u_{\mathfrak{M}}\right)_{\mathfrak{M}}+\llbracket \dot{e}_{\partial \mathfrak{M}}^{c, n+1}, u_{\partial \mathfrak{M}} \rrbracket_{1, \partial \mathfrak{M}}+\left(\frac{\dot{e}_{\partial \mathfrak{M}}^{c, n+1}-\dot{e}_{\partial \mathfrak{M}}^{c, n}}{\Delta t}, u_{\partial \mathfrak{M}}\right)_{\partial \mathfrak{M}} \\
= & \left(\bar{e}_{\mathfrak{M}}^{\mu, n+1}, u_{\mathfrak{M}}\right)_{L^{2}(\Omega)}+\left(R_{c_{1 \Gamma}}^{n+1}, u_{\partial \mathfrak{M}}\right)_{L^{2}(\Gamma)}-\left(\frac{\bar{e}_{\partial \mathfrak{M}}^{c, n+1}-\bar{e}_{\partial \mathfrak{M}}^{c, n}}{\Delta t}, u_{\partial \mathfrak{M}}\right)_{L^{2}(\Omega)} \\
& -\left(f_{b}^{\prime}\left(c\left(t^{n+1}, \cdot\right)\right)-\frac{m_{\mathfrak{M}}}{m_{\mathfrak{M}}} d^{f_{b}}\left(c_{\mathfrak{M}}^{n}, c_{\mathfrak{M}}^{n+1}\right), u_{\mathfrak{M}}\right)_{L^{2}(\Omega)} \\
& -\left(f_{s}^{\prime}\left(c_{\mid \Gamma}\left(t^{n+1}, \cdot\right)-\frac{m_{\underline{\partial M}}}{m_{\partial \mathfrak{M}}} d^{f_{s}}\left(c_{\partial \mathfrak{M}}^{n}, c_{\partial \mathfrak{M}}^{n+1}\right), u_{\partial \mathfrak{M}}\right)_{L^{2}(\Gamma)}\right.
\end{aligned}
$$

By choosing $v_{\mathcal{T}}=\Delta t \dot{e}_{\mathcal{T}}^{\mu, n+1}$ in equation (4.14) and $u_{\mathcal{T}}=\dot{e}_{\mathcal{T}}^{c, n+1}-\dot{e}_{\mathcal{T}}^{c, n}$ in equation (4.15) and adding the two resulting equalities the claim follows.

Proposition 4.14. Let $(c, \mu)$ be solution to the Cahn-Hilliard equation (1.1) and $\left(c_{\mathcal{T}}^{n+1}, \mu_{\mathcal{T}}^{n+1}\right)$ be solution to the discrete scheme (3.1). Then, for any $n_{0} \in \llbracket 0, N \rrbracket$ we have

$$
\begin{array}{r}
\frac{1}{2} \sum_{n=0}^{n_{0}} \Delta t\left|\dot{e}_{\mathcal{T}}^{\mu, n+1}\right|_{1, \mathcal{T}}^{2}+\frac{1}{2}\left(\frac{1}{2}\left|\dot{e}_{\mathcal{T}}^{c, n}+1\right|_{1, \mathcal{T}}^{2}-\left|\dot{e}_{\mathcal{T}}^{c, 0}\right|_{1, \mathcal{T}}^{2}+\sum_{n=0}^{n_{0}}\left|\dot{e}_{\mathcal{T}}^{c, n+1}-\dot{e}_{\mathcal{T}}^{c, n}\right|_{1, \mathcal{T}}^{2}\right) \\
+\frac{1}{2} \sum_{n=0}^{n_{0}} \Delta t \|\left.\frac{\dot{e}_{\partial \mathfrak{M}}^{c, n+1}-\dot{e}_{\partial \mathfrak{M}}^{c, n}}{\Delta t}\right|_{0, \partial \mathfrak{M}} ^{2}+\frac{1}{2}\left(\left|\dot{e}_{\partial \mathfrak{M}}^{c, n_{0}+1}\right|_{1, \partial \mathfrak{M}}^{2}-\left|\dot{e}_{\partial \mathfrak{M}}^{c, 0}\right|_{1, \partial \mathfrak{M}}^{2}+\sum_{n=0}^{n_{0}}\left|\dot{e}_{\partial \mathfrak{M}}^{c, n+1}-\dot{e}_{\partial \mathfrak{M}}^{c, n}\right|_{1, \partial \mathfrak{M}}^{2}\right) \\
\leq T_{c^{0}}+\sum_{n=1}^{n_{0}} \Delta t\left|\dot{e}_{\mathcal{T}}^{c, n}\right|_{1, \mathcal{T}}^{2}+T_{R_{c}^{n+1}}+T_{\bar{e}_{\mathcal{T}}}+T_{g_{\mathcal{T}}}+T_{f_{b}}+T_{f_{s}} \\
+\frac{(T+1) C_{5}^{2}}{2}\left(M_{1}+\left(C_{15}+1\right)\|c\|_{L^{\infty}\left(0, T ; H_{\Gamma}^{2}(\Omega)\right)}\right) \operatorname{size}(\mathcal{T})^{2},
\end{array}
$$


where the different error terms are defined as follows

$$
\begin{aligned}
T_{c^{0}}= & \left\|\dot{e}_{\mathfrak{M}}^{c, 0}\right\|_{L^{2}(\Omega)}^{2}+\frac{|\Omega|(T+1)}{2 C_{1}^{2}} m_{\underline{\Omega}}\left(\dot{e}_{\mathfrak{M}}^{c, 0}\right)^{2}, \\
T_{R_{c}^{n+1}}= & 3 C_{4}^{2} \sum_{n=0}^{n_{0}} \Delta t\left\|R_{c}^{n+1}\right\|_{L^{2}(\Omega)}^{2}+2 C_{3} \sum_{n=0}^{n_{0}} \Delta t\left\|R_{c_{\mid \Gamma \Gamma}}^{n+1}\right\|_{L^{2}(\Gamma)}^{2}, \\
T_{\bar{e}_{\mathcal{T}}=}= & 3 C_{4}^{2} \sum_{n=0}^{n_{0}} \Delta t\left\|\frac{\bar{e}_{\mathfrak{M}}^{c, n+1}-\bar{e}_{\mathfrak{M}}^{c, n}}{\Delta t}\right\|_{L^{2}(\Omega)}^{2}+2 C_{3} \sum_{n=0}^{n_{0}} \Delta t\left\|\frac{\bar{e}_{\partial \mathfrak{M}}^{c, n+1}-\bar{e}_{\partial \mathfrak{M}}^{c, n}}{\Delta t}\right\|_{L^{2}(\Gamma)}^{2} \\
& +\frac{1}{2}\left\|\bar{e}_{\mathfrak{M}}^{\mu, 1}\right\|_{L^{2}(\Omega)}^{2}+2 C_{4}^{2}\left\|\bar{e}_{\mathfrak{M}}^{\mu, n_{0}+1}\right\|_{L^{2}(\Omega)}^{2}+\frac{C_{4}^{2}}{2} \sum_{n=1}^{n_{0}} \Delta t\left\|\frac{\bar{e}_{\mathfrak{M}}^{\mu, n}-\bar{e}_{\mathfrak{M}}^{\mu, n+1}}{\Delta t}\right\|_{L^{2}(\Omega)}^{2}, \\
T_{g_{\mathcal{T}}=} & \frac{1}{2}\left\|g_{\mathfrak{M}}\left(t^{1}, \cdot\right)\right\|_{L^{2}(\Omega)}^{2}+\left(2 C_{4}^{2}+1\right)\left\|g_{\mathfrak{M}}\left(t^{n_{0}+1}, \cdot\right)\right\|_{L^{2}(\Omega)}^{2} \\
& +\left(\frac{C_{4}^{2}}{2}+1\right) \sum_{n=1}^{n_{0}} \Delta t\left\|\frac{g_{\mathfrak{M}}\left(t^{n}, \cdot\right)-g_{\mathfrak{M}}\left(t^{n+1}, \cdot\right)}{\Delta t}\right\|_{L^{2}(\Omega)}^{2}+2 C_{3} \sum_{n=0}^{n_{0}} \Delta t\left\|g_{\partial \mathfrak{M}}\left(t^{n+1}, \cdot\right)\right\|_{L^{2}(\Gamma)}^{2}, \\
T_{f_{b}}= & 3 \sum_{n=0}^{n_{0}} \Delta t\left(\left|f_{b}^{\prime}\left(\mathbb{P}_{\mathcal{T}}^{c}\left(c\left(t^{n+1}\right)\right)\right)-f_{b}^{\prime}\left(c_{\mathcal{T}}^{n+1}\right)\right|_{1, \mathcal{T}}^{2}+\left|d^{f_{b}}\left(c_{\mathcal{T}}^{n+1}, c_{\mathcal{T}}^{n+1}\right)-d^{f_{b}}\left(c_{\mathcal{T}}^{n}, c_{\mathcal{T}}^{n+1}\right)\right|_{1, \mathcal{T}}^{2}\right), \\
T_{f_{s}}= & 2 \sum_{n=0}^{n_{0}} \Delta t\left\|f_{s}^{\prime}\left(\mathbb{P}_{\partial \mathfrak{M}}^{c}\left(c\left(t^{n+1}\right)\right)\right)-d^{f_{s}}\left(c_{\partial \mathfrak{M}}^{n}, c_{\partial \mathfrak{M}}^{n+1}\right)\right\|_{L^{2}(\Gamma)}^{2} \cdot
\end{aligned}
$$

The term $g_{\mathfrak{M}}$ (resp. $\left.g_{\partial \mathfrak{M}}\right)$ is such that for any $\mathcal{K} \in \mathfrak{M}($ resp. $\mathcal{L} \in \partial \mathfrak{M})$,

$$
\begin{aligned}
g_{\mathfrak{M}}(t, x) & =f_{b}^{\prime}(c(t, x))-\frac{m_{\underline{\mathcal{K}}}}{m_{\mathcal{K}}} f_{b}^{\prime}\left(c\left(t, x_{\mathcal{K}}\right)\right), \quad \forall x \in \mathcal{K}, t \in \mathbb{R} ; \\
g_{\partial \mathfrak{M}}(t, x) & =f_{s}^{\prime}\left(c_{\mid \Gamma}(t, x)\right)-\frac{m_{\mathbf{e}_{\mathcal{L}}}}{m_{\mathcal{L}}} f_{s}^{\prime}\left(c_{\mid \Gamma}\left(t, x_{\mathcal{L}}\right)\right), \quad \forall x \in \mathcal{L}, t \in \mathbb{R} .
\end{aligned}
$$

Proof. To begin we sum identity (4.6) for $n$ going from 0 to $n_{0}$ and we use definition (4.17) of the functions $g_{\mathfrak{M}}$ and $g_{\partial \mathfrak{M}}$ for the terms where the nonlinear potentials appear. To obtain estimate (4.16), we have to apply the Young inequality to all the terms in the right-hand side of the resulting equality.

- Let us begin by the terms where the $L^{2}$-inner product with $\dot{e}_{\mathfrak{M}}^{\mu, n+1}$ appears. Noting that if $v_{\mathfrak{M}} \in \mathbb{R}^{\mathfrak{M}}$ has a zero mean-value, for any $u_{\mathfrak{M}} \in \mathbb{R}^{\mathfrak{M}}$, we have

$$
\left(u_{\mathfrak{M}}, v_{\mathfrak{M}}\right)_{L^{2}(\Omega)}=\left(u_{\mathfrak{M}}-m_{\Omega}\left(u_{\mathfrak{M}}\right), v_{\mathfrak{M}}\right)_{L^{2}(\Omega)} \leq\left\|u_{\mathfrak{M}}-m_{\Omega}\left(u_{\mathfrak{M}}\right)\right\|_{L^{2}(\Omega)}\left\|v_{\mathfrak{M}}\right\|_{L^{2}(\Omega)},
$$

then thanks to the discrete mean Poincaré inequality given in Lemma 2.7, we obtain

$$
\left(u_{\mathfrak{M}}, v_{\mathfrak{M}}\right)_{L^{2}(\Omega)} \leq C_{4}\left|u_{\mathcal{T}}\right|_{1, \mathcal{T}}\left\|v_{\mathfrak{M}}\right\|_{L^{2}(\Omega)} .
$$

The couple $(c, \mu)$ is solution to the Cahn-Hilliard equation (1.1) with the homogeneous Neumann boundary condition for $\mu$ so for any $n \in \llbracket 0, n_{0} \rrbracket, m_{\Omega}\left(R_{c}^{n+1}\right)=0$. In the same way, thanks to Definition 4.7 of the elliptic projection, for any $n \in \llbracket 0, n_{0} \rrbracket$ we have $m_{\Omega}\left(\bar{e}_{\mathfrak{M}}^{c, n}\right)=0$ and so $m_{\Omega}\left(\bar{e}_{\mathfrak{M}}^{c, n+1}-\bar{e}_{\mathfrak{M}}^{c, n}\right)=0$. Thus, owing to estimate (4.18) and the Young inequality, for any $n \in \llbracket 0, n_{0} \rrbracket$ one has

$$
\Delta t\left(R_{c}^{n+1}, \dot{e}_{\mathfrak{M}}^{\mu, n+1}\right)_{L^{2}(\Omega)} \leq \frac{1}{8} \Delta t\left|\dot{e}_{\mathcal{T}}^{\mu, n+1}\right|_{1, \mathcal{T}}^{2}+2 C_{4}^{2} \Delta t\left\|R_{c}^{n+1}\right\|_{L^{2}(\Omega)}^{2}
$$

and

$$
\left(\bar{e}_{\mathfrak{M}}^{c, n+1}-\bar{e}_{\mathfrak{M}}^{c, n}, \dot{e}_{\mathfrak{M}}^{\mu, n+1}\right)_{L^{2}(\Omega)} \leq \frac{1}{8} \Delta t\left|\dot{e}_{\mathcal{T}}^{\mu, n+1}\right|_{1, \mathcal{T}}^{2}+2 C_{4}^{2} \Delta t\left\|\frac{\bar{e}_{\mathfrak{M}}^{c, n+1}-\bar{e}_{\mathfrak{M}}^{c, n}}{\Delta t}\right\|_{L^{2}(\Omega)}^{2}
$$


- Now we focus on the terms whose term $\dot{e}_{\mathfrak{m}}^{c, n+1}-\dot{e}_{\mathfrak{m}}^{c, n}$ appears in the inner product on $L^{2}(\Omega)$. For the two first we perform a discrete time integration by parts.

Let us begin by the term where the function $\bar{e}_{\mathfrak{M}}^{\mu, n+1}$ intervenes.

$$
\begin{aligned}
\sum_{n=0}^{n_{0}}\left(\bar{e}_{\mathfrak{M}}^{\mu, n+1}, \dot{e}_{\mathfrak{M}}^{c, n+1}-\dot{e}_{\mathfrak{M}}^{c, n}\right)_{L^{2}(\Omega)}= & -\left(\bar{e}_{\mathfrak{M}}^{\mu, 1}, \dot{e}_{\mathfrak{M}}^{c, 0}\right)_{L^{2}(\Omega)}+\left(\bar{e}_{\mathfrak{M}}^{\mu, n_{0}+1}, \dot{e}_{\mathfrak{M}}^{c, n_{0}+1}\right)_{L^{2}(\Omega)} \\
& +\sum_{n=1}^{n_{0}}\left(\bar{e}_{\mathfrak{M}}^{\mu, n}-\bar{e}_{\mathfrak{M}}^{\mu, n+1}, \dot{e}_{\mathfrak{M}}^{c, n}\right)_{L^{2}(\Omega)} .
\end{aligned}
$$

Noting that by definition of the elliptic projection (see Definition 4.6) $m_{\Omega}\left(\bar{e}_{\mathfrak{m}}^{\mu, n}\right)=0$, the Cauchy-Schwartz inequality, inequality (4.18) and the Young inequality give

$$
\begin{aligned}
& \left|\sum_{n=0}^{n_{0}}\left(\bar{e}_{\mathfrak{M}}^{\mu, n+1}, \dot{e}_{\mathfrak{M}}^{c, n+1}-\dot{e}_{\mathfrak{M}}^{c, n}\right)_{L^{2}(\Omega)}\right| \leq \frac{1}{2}\left\|\bar{e}_{\mathfrak{M}}^{\mu, 1}\right\|_{L^{2}(\Omega)}^{2}+\frac{1}{2}\left\|\dot{e}_{\mathfrak{M}}^{c, 0}\right\|_{L^{2}(\Omega)}^{2} \\
& \quad+\frac{C_{4}^{2}}{2} \sum_{n=1}^{n_{0}} \Delta t\left\|\frac{\bar{e}_{\mathfrak{M}}^{\mu, n}-\bar{e}_{\mathfrak{M}}^{\mu, n+1}}{\Delta t}\right\|_{L^{2}(\Omega)}^{2}+\frac{1}{2} \sum_{n=1}^{n_{0}} \Delta t\left|\dot{e}_{\mathcal{T}}^{c, n}\right|_{1, \mathcal{T}}^{2} \\
& \quad+\frac{1}{8}\left|\dot{e}_{\mathcal{T}}^{c, n_{0}+1}\right|_{1, \mathcal{T}}^{2}+2 C_{4}^{2}\left\|\bar{e}_{\mathfrak{M}}^{\mu, n_{0}+1}\right\|_{L^{2}(\Omega)}^{2} .
\end{aligned}
$$

Considering now the inner product with the term $g_{\mathfrak{M}}\left(t^{n+1}, \cdot\right)$. Since the mean-value of the function $g_{\mathfrak{M}}(t, \cdot)$ is not equal to 0 , we cannot apply exactly the same reasoning. Definition 4.7 of the elliptic projection and the discrete volume conservation (3.3) imply that for any $n \in \llbracket 0, N \rrbracket, m_{\underline{\Omega}}\left(\dot{e}_{\mathfrak{m}}^{c, n}\right)=m_{\underline{\Omega}}\left(\dot{e}_{\mathfrak{m}}^{c, 0}\right)$. Then the Cauchy-Schwarz inequality, Proposition 2.1, Poincaré inequality (2.3) and the Young inequality get

$$
\begin{aligned}
\left|\sum_{n=0}^{n_{0}}\left(g_{\mathfrak{M}}\left(t^{n+1}, \cdot\right), \dot{e}_{\mathfrak{M}}^{c, n+1}-\dot{e}_{\mathfrak{M}}^{c, n}\right)_{L^{2}(\Omega)}\right| \leq \frac{1}{2}\left\|g_{\mathfrak{M}}\left(t^{1}, \cdot\right)\right\|_{L^{2}(\Omega)}^{2}+\frac{1}{2}\left\|\dot{e}_{\mathfrak{M}}^{c, 0}\right\|_{L^{2}(\Omega)}^{2} \\
\quad+\left(2 C_{4}^{2}+1\right)\left\|g_{\mathfrak{M}}\left(t^{n_{0}+1}, \cdot\right)\right\|_{L^{2}(\Omega)}^{2}+\frac{1}{8}\left|\dot{e}_{\mathcal{T}}^{c, n_{0}+1}\right|_{1, \mathcal{T}}^{2}+\frac{|\Omega|(T+1)}{2 C_{1}^{2}} m_{\underline{\Omega}}\left(\dot{e}_{\mathfrak{M}}^{c, 0}\right)^{2} \\
\quad+\frac{1}{2} \sum_{n=1}^{n_{0}} \Delta t\left|\dot{e}_{\mathcal{T}}^{c, n}\right|_{1, \mathcal{T}}^{2}+\left(\frac{C_{4}^{2}}{2}+1\right) \sum_{n=1}^{n_{0}} \Delta t\left\|\frac{g_{\mathfrak{M}}\left(t^{n}, \cdot\right)-g_{\mathfrak{M}}\left(t^{n+1}, \cdot\right)}{\Delta t}\right\|_{L^{2}(\Omega)}^{2} \\
\quad+\frac{C_{5}^{2}}{2} \operatorname{size}(\mathcal{T})^{2}\left(\left\|\dot{e}_{\mathfrak{M}}^{c, n_{0}+1}\right\|_{L^{2}(\Omega)}^{2}+\sum_{n=1}^{n_{0}} \Delta t\left\|\dot{e}_{\mathcal{T}}^{c, n}\right\|_{L^{2}(\Omega)}^{2}\right) .
\end{aligned}
$$

Thanks to the bounds on the discrete solutions (Proposition 4.5) and Proposition 4.11, for any $n \in \llbracket 0, N \rrbracket$, one has

$$
\left\|\dot{e}_{\mathcal{T}}^{c, n}\right\|_{L^{2}(\Omega)} \leq M_{1}+\left(C_{15}+1\right)\|c\|_{L^{\infty}\left(0, T ; H_{\Gamma}^{2}(\Omega)\right)} .
$$

As regards the last term where the inner product with $\dot{e}_{\mathfrak{M}}^{c, n+1}-\dot{e}_{\mathfrak{m}}^{c, n}$ appears we have to use the scheme. Choosing $v_{\mathcal{T}}=\Delta t\left(f_{b}^{\prime}\left(\mathbb{P}_{\mathcal{T}}^{c}\left(c\left(t^{n+1}\right)\right)\right)-d^{f_{b}}\left(c_{\mathcal{T}}^{n}, c_{\mathcal{T}}^{n+1}\right)\right)$ in identity (4.14) we obtain

$$
\begin{aligned}
&\left(f_{b}^{\prime}\left(\mathbb{P}_{\mathfrak{M}}^{c}\left(c\left(t^{n+1}\right)\right)\right)-d^{f_{b}}\left(c_{\mathfrak{M}}^{n}, c_{\mathfrak{M}}^{n+1}\right), \dot{e}_{\mathfrak{M}}^{c, n+1}-\dot{e}_{\mathfrak{M}}^{c, n}\right)_{\mathfrak{M}} \\
&=-\Delta t \llbracket \dot{e}_{\mathcal{T}}^{\mu, n+1}, f_{b}^{\prime}\left(\mathbb{P}_{\mathcal{T}}^{c}\left(c\left(t^{n+1}\right)\right)\right)-d^{f_{b}}\left(c_{\mathcal{T}}^{n}, c_{\mathcal{T}}^{n+1}\right) \rrbracket_{1, \mathcal{T}} \\
&+\Delta t\left(R_{c}^{n+1}, f_{b}^{\prime}\left(\mathbb{P}_{\mathfrak{M}}^{c}\left(c\left(t^{n+1}\right)\right)\right)-d^{f_{b}}\left(c_{\mathfrak{M}}^{n}, c_{\mathfrak{M}}^{n+1}\right)\right)_{L^{2}(\Omega)} \\
&-\left(\bar{e}_{\mathfrak{M}}^{c, n+1}-\bar{e}_{\mathfrak{M}}^{c, n}, f_{b}^{\prime}\left(\mathbb{P}_{\mathfrak{M}}^{c}\left(c\left(t^{n+1}\right)\right)\right)-d^{f_{b}}\left(c_{\mathfrak{M}}^{n}, c_{\mathfrak{M}}^{n+1}\right)\right)_{L^{2}(\Omega)} .
\end{aligned}
$$


Since $m_{\Omega}\left(R_{c}^{n+1}\right)=m_{\Omega}\left(\bar{e}_{\mathfrak{M}}^{c, n+1}-\bar{e}_{\mathfrak{M}}^{c, n}\right)=0$, the Cauchy-Schwarz inequality and inequality (4.18) imply

$$
\begin{aligned}
& \left(f_{b}^{\prime}\left(\mathbb{P}_{\mathfrak{M}}^{c}\left(c\left(t^{n+1}\right)\right)\right)-\frac{m_{\mathfrak{M}}}{m_{\mathfrak{M}}} d^{f_{b}}\left(c_{\mathfrak{M}}^{n}, c_{\mathfrak{M}}^{n+1}\right), \dot{e}_{\mathfrak{M}}^{c, n+1}-\dot{e}_{\mathfrak{M}}^{c, n}\right)_{L^{2}(\Omega)} \\
& \leq \Delta t\left(\left|\dot{e}_{\mathcal{T}}^{\mu, n+1}\right|_{1, \mathcal{T}}+C_{4}\left\|R_{c}^{n+1}\right\|_{L^{2}(\Omega)}+C_{4}\left\|\frac{\bar{e}_{\mathfrak{M}}^{c, n+1}-\bar{e}_{\mathfrak{M}}^{c, n}}{\Delta t}\right\|_{L^{2}(\Omega)}\right) \\
& \quad \times\left|f_{b}^{\prime}\left(\mathbb{P}_{\mathcal{T}}^{c}\left(c\left(t^{n+1}\right)\right)\right)-d^{f_{b}}\left(c_{\mathcal{T}}^{n}, c_{\mathcal{T}}^{n+1}\right)\right|_{1, \mathcal{T}} .
\end{aligned}
$$

Recalling that for any $x \in \mathbb{R}, d^{f_{b}}(x, x)=f_{b}^{\prime}(x)$ (see Definition 3.1) and applying the Young inequality we deduce

$$
\begin{aligned}
& \left(f_{b}^{\prime}\left(\mathbb{P}_{\mathfrak{M}}^{c}\left(c\left(t^{n+1}\right)\right)\right)-d^{f_{b}}\left(c_{\mathfrak{M}}^{n}, c_{\mathfrak{M}}^{n+1}\right), \dot{e}_{\mathfrak{M}}^{c, n+1}-\dot{e}_{\mathfrak{M}}^{c, n}\right)_{\mathfrak{M}} \\
& \quad \leq \frac{1}{4} \Delta t\left|\dot{e}_{\mathcal{T}}^{\mu, n+1}\right|_{1, \mathcal{T}}^{2}+C_{4}^{2} \Delta t\left(\left\|R_{c}^{n+1}\right\|_{L^{2}(\Omega)}^{2}+\left\|\frac{\bar{e}_{\mathfrak{M}}^{c, n+1}-\bar{e}_{\mathfrak{M}}^{c, n}}{\Delta t}\right\|_{L^{2}(\Omega)}^{2}\right) \\
& \quad+3 \Delta t\left|f_{b}^{\prime}\left(\mathbb{P}_{\mathcal{T}}^{c}\left(c\left(t^{n+1}\right)\right)\right)-f_{b}^{\prime}\left(c_{\mathcal{T}}^{n+1}\right)\right|_{1, \mathcal{T}}^{2} \\
& \quad+3 \Delta t\left|d^{f_{b}}\left(c_{\mathcal{T}}^{n+1}, c_{\mathcal{T}}^{n+1}\right)-d^{f_{b}}\left(c_{\mathcal{T}}^{n}, c_{\mathcal{T}}^{n+1}\right)\right|_{1, \mathcal{T}}^{2} .
\end{aligned}
$$

- Finally we focus on the terms due to the dynamic boundary condition, that is the terms where the inner product in $L^{2}(\Gamma)$ appears.

According to the Cauchy-Schwarz inequality, the Young inequality and the equivalence of norms $\|\cdot\|_{0, \partial \mathfrak{M}}$ and $\|\cdot\|_{L^{2}(\Gamma)}$, for any $u_{\partial \mathfrak{M}} \in \mathbb{R}^{\partial \mathfrak{M}}$ we have

$$
\left(u_{\partial \mathfrak{M}}, \dot{e}_{\partial \mathfrak{M}}^{c, n+1}-\dot{e}_{\partial \mathfrak{M}}^{c, n}\right)_{L^{2}(\Gamma)} \leq \frac{1}{8} \Delta t\left\|\frac{\dot{e}_{\partial \mathfrak{M}}^{c, n+1}-\dot{e}_{\partial \mathfrak{M}}^{c, n}}{\Delta t}\right\|_{0, \partial \mathfrak{M}}^{2}+2 C_{3} \Delta t\left\|u_{\partial \mathfrak{M}}\right\|_{L^{2}(\Gamma)}^{2} .
$$

Choosing $u_{\partial \mathfrak{M}}=R_{c_{1 \Gamma} \Gamma}^{n+1}$, then $u_{\partial \mathfrak{M}}=\frac{\bar{e}_{\partial \mathfrak{M}}^{c, n+1}-\bar{e}_{\partial \mathfrak{M}}^{c, n}}{\Delta t}$ and finally $u_{\partial \mathfrak{M}}=g_{\partial \mathfrak{M}}\left(t^{n+1}, \cdot\right)$ in equation (4.25) we can control the first three terms.

For the last term, setting $u_{\partial \mathfrak{M}}=f_{s}^{\prime}\left(\mathbb{P}_{\partial \mathfrak{M}}^{c}\left(c\left(t^{n+1}\right)\right)\right)-d^{f_{s}}\left(c_{\partial \mathfrak{M}}^{n}, c_{\partial \mathfrak{M}}^{n+1}\right)$ in the same way as for equation (4.25) one has

$$
\begin{aligned}
& -\left(f_{s}^{\prime}\left(\mathbb{P}_{\partial \mathfrak{M}}^{c}\left(c\left(t^{n+1}\right)\right)\right)-d^{f_{s}}\left(c_{\partial \mathfrak{M}}^{n}, c_{\partial \mathfrak{M}}^{n+1}\right), \dot{e}_{\partial \mathfrak{M}}^{c, n+1}-\dot{e}_{\partial \mathfrak{M}}^{c, n}\right)_{\partial \mathfrak{M}} \\
& \leq \frac{1}{8} \Delta t\left\|\frac{\dot{e}_{\partial \mathfrak{M}}^{c, n+1}-\dot{e}_{\partial \mathfrak{M}}^{c, n}}{\Delta t}\right\|_{0, \partial \mathfrak{M}}^{2}+2 \Delta t\left\|f_{s}^{\prime}\left(\mathbb{P}_{\partial \mathfrak{M}}^{c}\left(c\left(t^{n+1}\right)\right)\right)-d^{f_{s}}\left(c_{\partial \mathfrak{M}}^{n}, c_{\partial \mathfrak{M}}^{n+1}\right)\right\|_{L^{2}(\Gamma)}^{2}
\end{aligned}
$$

Gathering inequalities (4.19)-(4.26), we obtain estimate (4.16).

4.3.2. Estimate of the residual terms. In order to apply the discrete Gronwall lemma, we have to estimate all the terms in the right-hand side of inequality (4.16) independently of $n$.

We begin by a bound on the initial data.

Proposition 4.15. Let $c^{0} \in \mathcal{C}^{2}(\bar{\Omega})$ and $c_{\mathcal{T}}^{0}=\mathbb{P}_{\mathcal{T}}^{c} c^{0}$. Then for some $C_{16}>0$ we have

$$
m_{\Omega}\left(\dot{e}_{\mathfrak{M}}^{c, 0}\right)+m_{\underline{\Omega}}\left(\dot{e}_{\mathfrak{M}}^{c, 0}\right)+\left\|\dot{e}_{\mathfrak{M}}^{c, 0}\right\|_{L^{2}(\Omega)}+\left|\dot{e}_{\mathcal{T}}^{c, 0}\right|_{1, \mathcal{T}}+\left|\dot{e}_{\partial \mathfrak{M}}^{c, 0}\right|_{1, \partial \mathfrak{M}} \leq C_{16} \operatorname{size}(\mathcal{T})\left\|c^{0}\right\|_{H_{\Gamma}^{2}(\Omega)}
$$


Proof. Estimates of the (semi)norms are only a direct consequence of the definition of $c_{\mathcal{T}}^{0}$ and Proposition 4.11 (associated with Lemma 4.9 for the $L^{2}$-norm).

Then owing to Proposition 2.1 and the Cauchy-Schwarz inequality we deduce

$$
m_{\underline{\Omega}}\left(\dot{e}_{\mathfrak{M}}^{c, 0}\right) \leq\left(\frac{C_{2}}{C_{1}|\Omega|}\right)^{\frac{1}{2}}\left\|\dot{e}_{\mathfrak{M}}^{c, 0}\right\|_{L^{2}(\Omega)} \text { and } m_{\Omega}\left(\dot{e}_{\mathfrak{M}}^{c, 0}\right) \leq \frac{1}{|\Omega|^{\frac{1}{2}}}\left\|\dot{e}_{\mathfrak{M}}^{c, 0}\right\|_{L^{2}(\Omega)},
$$

and the $L^{2}$-estimate gives the claim.

Applying the Taylor's theorem to the term $R_{c}^{n+1}$ defined by (4.7) we can easily obtain the following estimates on the term $T_{R_{c}^{n+1}}$ about the error of the time discretization.

Proposition 4.16. For any $n_{0} \in \llbracket 0, N \rrbracket$, the remainder terms $R_{c}^{n+1}$ and $R_{c_{1 \Gamma}}^{n+1}$ defined by (4.7) satisfy

$$
\begin{aligned}
& \sum_{n=0}^{n_{0}} \Delta t\left\|R_{c}^{n+1}\right\|_{L^{2}(\Omega)}^{2} \leq\left\|\partial_{t t} c\right\|_{L^{2}\left(0, T ; L^{2}(\Omega)\right)}^{2} \Delta t^{2}, \\
& \sum_{n=0}^{n_{0}} \Delta t\left\|R_{c_{\mid \Gamma}}^{n+1}\right\|_{L^{2}(\Gamma)}^{2} \leq\left\|\partial_{t t} c_{\mid \Gamma}\right\|_{L^{2}\left(0, T ; L^{2}(\Gamma)\right)}^{2} \Delta t^{2} .
\end{aligned}
$$

Now we are interested in the term $T_{\bar{e}_{\mathcal{T}}}$ concerning the error between the exact solution and its elliptic projection.

Proposition 4.17. There exists $C_{17}>0$ such that for any $n_{0} \in \llbracket 0, N \rrbracket$ the following estimates hold

$$
\begin{aligned}
& \sum_{n=0}^{n_{0}} \Delta t\left\|\frac{\bar{e}_{\mathfrak{M}}^{c, n+1}-\bar{e}_{\mathfrak{M}}^{c, n}}{\Delta t}\right\|_{L^{2}(\Omega)}^{2} \leq C_{17}^{2} \operatorname{size}(\mathcal{T})^{2}\left\|\partial_{t} c\right\|_{L^{2}\left(0, T ; H_{\Gamma}^{2}(\Omega)\right)}^{2}, \\
& \sum_{n=0}^{n_{0}} \Delta t\left\|\frac{\bar{e}_{\partial \mathfrak{M}}^{c, n+1}-\bar{e}_{\partial \mathfrak{M}}^{c, n}}{\Delta t}\right\|_{L^{2}(\Gamma)}^{2} \leq C_{17}^{2} \operatorname{size}(\mathcal{T})^{2}\left\|\partial_{t} c\right\|_{L^{2}\left(0, T ; H_{\Gamma}^{2}(\Omega)\right)}^{2}, \\
& \sum_{n=0}^{n_{0}} \Delta t\left\|\frac{\bar{e}_{\mathfrak{M}}^{\mu, n+1}-\bar{e}_{\mathfrak{M}}^{\mu, n}}{\Delta t}\right\|_{L^{2}(\Omega)}^{2} \leq C_{17}^{2} \operatorname{size}(\mathcal{T})^{2}\left\|\partial_{t} \mu\right\|_{L^{2}\left(0, T ; H^{2}(\Omega)\right)}^{2} .
\end{aligned}
$$

Moreover, for any $n \in \llbracket 0, N \rrbracket$ we have

$$
\left\|\bar{e}_{\mathfrak{M}}^{\mu, n}\right\|_{L^{2}(\Omega)} \leq C_{17} \operatorname{size}(\mathcal{T})\|\mu\|_{L^{\infty}\left(0, T ; H^{2}(\Omega)\right)} .
$$

Proof. We begin by proving estimates (4.28). Thanks to Definition 4.12 we have

$$
\begin{aligned}
\left\|\bar{e}_{\mathfrak{M}}^{c, n+1}-\bar{e}_{\mathfrak{M}}^{c, n}\right\|_{L^{2}(\Omega)}^{2} \leq & 2\left\|\left(c\left(t^{n+1}, \cdot\right)-c\left(t^{n}, \cdot\right)\right)-\left(\mathbb{P}_{\mathfrak{M}}^{\mathrm{ell}, \mathrm{D}} c\left(t^{n+1}, \cdot\right)-\mathbb{P}_{\mathfrak{M}}^{\mathrm{ell}, \mathrm{D}} c\left(t^{n}, \cdot\right)\right)\right\|_{L^{2}(\Omega)}^{2} \\
& +2 \sum_{\mathcal{K} \in \mathfrak{M}} \int_{\mathcal{K}}\left(\frac{m_{\mathcal{K}}-m_{\underline{\mathcal{K}}}}{m_{\mathcal{K}}}\right)^{2}\left(\mathbb{P}_{\mathcal{K}}^{\mathrm{ell}, \mathrm{D}} c\left(t^{n+1}, \cdot\right)-\mathbb{P}_{\mathcal{K}}^{\mathrm{ell}, \mathrm{D}} c\left(t^{n}, \cdot\right)\right)^{2}
\end{aligned}
$$

Owing to the linearity of the elliptic projection (see Definition 4.7) and Proposition 4.11, the first term in the right-hand side satisfies

$$
\begin{aligned}
\|\left(c\left(t^{n+1}, \cdot\right)-c\left(t^{n}, \cdot\right)\right)-\left(\mathbb{P}_{\mathfrak{M}}^{\mathrm{ell}, \mathrm{D}} c\left(t^{n+1}, \cdot\right)\right. & \left.-\mathbb{P}_{\mathfrak{M}}^{\mathrm{ell}, \mathrm{D}} c\left(t^{n}, \cdot\right)\right) \|_{L^{2}(\Omega)}^{2} \\
& \leq C_{15}^{2} \Delta t \operatorname{size}(\mathcal{T})^{2} \int_{t^{n}}^{t^{n+1}}\left\|\partial_{t} c(t, \cdot)\right\|_{H_{\Gamma}^{2}(\Omega)}^{2} \mathrm{~d} t
\end{aligned}
$$


Moreover, thanks to Proposition 2.1 and the mesh regularity (2.1) for any $\mathcal{K} \in \mathfrak{M}$ we have $m_{\mathcal{K}}-m_{\underline{\mathcal{K}}} \leq C \operatorname{reg}(\mathcal{T})^{2} m_{\mathcal{K}} \operatorname{diam}(\mathcal{K})$. Thus Proposition 4.11 yields

$$
\begin{aligned}
\sum_{\mathcal{K} \in \mathfrak{M}} \int_{\mathcal{K}} & \left(\frac{m_{\mathcal{K}}-m_{\underline{\mathcal{K}}}}{m_{\mathcal{K}}}\right)^{2}\left(\mathbb{P}_{\mathcal{K}}^{\mathrm{ell}, \mathrm{D}} c\left(t^{n+1}, \cdot\right)-\mathbb{P}_{\mathcal{K}}^{\mathrm{pell}, \mathrm{D}} c\left(t^{n}, \cdot\right)\right)^{2} \\
& \leq 2 C(\operatorname{reg}(\mathcal{T}))\left(C_{15}^{2}+1\right) \Delta t \operatorname{size}(\mathcal{T})^{2} \int_{t^{n}}^{t^{n+1}}\left\|\partial_{t} c(t, \cdot)\right\|_{H_{\Gamma}^{2}(\Omega)}^{2} \mathrm{~d} t .
\end{aligned}
$$

Summing inequalities (4.30) and (4.31) for $n$ going from 0 to $n_{0}$ provides estimate (4.28a).

Estimate (4.28c) is obtained with exactly the same reasoning using Definition 4.6 of the elliptic projection (instead of Definition 4.7) and Proposition 4.10 (instead of Proposition 4.11).

In the same way we deduce estimate (4.28b) with a similar reasoning on $\Gamma$ and Proposition 2.2 instead of Proposition 2.1.

It remains to prove estimate (4.29). For any $n \in \llbracket 0, N \rrbracket$,

$$
\left\|\bar{e}_{\mathfrak{M}}^{\mu, n}\right\|_{L^{2}(\Omega)}^{2} \leq 2\left\|\mu\left(t^{n}, \cdot\right)-\mathbb{P}_{\mathfrak{M}}^{\mathrm{ell}, \mathrm{N}} \mu\left(t^{n}, \cdot\right)\right\|_{L^{2}(\Omega)}^{2}+2 \sum_{\mathcal{K} \in \mathfrak{M}} \int_{\mathcal{K}}\left(\frac{m_{\mathcal{K}}-m_{\underline{\mathcal{K}}}}{m_{\mathcal{K}}}\right)^{2}\left(\mathbb{P}_{\mathcal{K}}^{\mathrm{ell}, \mathrm{N}} \mu\left(t^{n}, \cdot\right)\right)^{2}
$$

Then, applying Proposition 4.10 to the function $\mu\left(t^{n}, \cdot\right)$ the first term in the right-hand side is written as follows

$$
\left\|\mu\left(t^{n}, \cdot\right)-\mathbb{P}_{\mathfrak{M}}^{\mathrm{ell}, \mathrm{N}} \mu\left(t^{n}\right)\right\|_{L^{2}(\Omega)} \leq C_{14} \operatorname{size}(\mathcal{T})\|\mu\|_{L^{\infty}\left(0, T ; H^{2}(\Omega)\right)} .
$$

The second term is treated as estimate (4.31) that concludes the proof.

We can now we concentrate on the error due to the discretization of nonlinear terms. In this way we begin with the term $T_{g_{\mathcal{T}}}$ related to the terms $g_{\mathfrak{M}}$ and $g_{\partial \mathfrak{M}}$.

Proposition 4.18. For any $t \in[0, T]$, the functions $g_{\mathfrak{m}}$ and $g_{\partial \mathfrak{m}}$ defined by (4.17) satisfy the following estimates with $C_{18}$ (respectively $C_{19}$ ) depending on $f_{b}$ (repectively $f_{s}$ ) but not on $\Delta t$ and $\operatorname{size}(\mathcal{T})$,

$$
\begin{aligned}
&\left\|g_{\mathfrak{M}}(t, \cdot)\right\|_{L^{2}(\Omega)}^{2} \leq C_{18} \operatorname{size}(\mathcal{T})^{2}\left(\|\nabla c(t, \cdot)\|_{H^{1}(\Omega)}^{2}+\|c(t, \cdot)\|_{L^{\infty}(\Omega)}^{2 p}+1\right) \\
&\left\|g_{\partial \mathfrak{M}}(t, \cdot)\right\|_{L^{2}(\Gamma)}^{2} \leq C_{19} \operatorname{size}(\mathcal{T})^{2}\left(\left\|\nabla_{\Gamma} c_{\mid \Gamma}(t, \cdot)\right\|_{L^{2}(\Gamma)}^{2}+\left(\max _{\bar{B}\left(0,\|c(t, \cdot)\|_{L^{\infty}(\Gamma)}\right)}^{2}\left|f_{s}^{\prime}\right|\right)^{2}\right) .
\end{aligned}
$$

Moreover, there exists $C_{20}>0$ depending only on $f_{b}$ (and on its derivatives) such that for any $n_{0} \in \llbracket 0, N \rrbracket$,

$$
\sum_{n=0}^{n_{0}} \Delta t\left\|\frac{g_{\mathfrak{M}}\left(t^{n+1}, \cdot\right)-g_{\mathfrak{M}}\left(t^{n}, \cdot\right)}{\Delta t}\right\|_{L^{2}(\Omega)}^{2} \leq C_{20} M_{\infty}^{c}\left\|\partial_{t} c\right\|_{L^{2}\left(0, T ; H^{2}(\Omega)\right)}^{2} \operatorname{size}(\mathcal{T})^{2}
$$

where

$$
M_{\infty}^{c}=\max \left(1,\|\nabla c\|_{L^{\infty}\left(0, T ; L^{\infty}(\Omega)\right)}^{4},\left\|D^{2} c\right\|_{L^{\infty}\left(0, T ; L^{\infty}(\Omega)\right)}^{2}\right) .
$$

Proof. Thanks to definition (4.17) of $g_{\mathfrak{M}}$ and $g_{\partial \mathfrak{m}}$ and since $f_{b}^{\prime}$ and $f_{s}^{\prime}$ are Lipschitz continuous functions, Propositions 2.1 and 2.2, the mesh regularity (2.1), the polynomial growth assumption (1.2) for $f_{b}$, the bounds on the discrete solutions in Proposition 4.5 and Lemma 4.9 yield estimates (4.32). 
We concentrate now on estimate (4.33). For any $n \in \llbracket 0, N-1 \rrbracket$ we define the function $h^{n}$ such that for any $x \in \Omega$,

$$
h^{n}(x)=f_{b}^{\prime}\left(c\left(t^{n+1}, x\right)\right)-f_{b}^{\prime}\left(c\left(t^{n}, x\right)\right) .
$$

Therefore, for any $\mathcal{K} \in \mathfrak{M}$ and for any $n \in \llbracket 0, N-1 \rrbracket$,

$$
g_{\mathfrak{M}}\left(t^{n+1}, x\right)-g_{\mathfrak{M}}\left(t^{n}, x\right)=h^{n}(x)-\frac{m_{\underline{\mathcal{K}}}}{m_{\mathcal{K}}} h^{n}\left(x_{\mathcal{K}}\right), \quad \forall x \in \mathcal{K} .
$$

Thus, thanks to Lemma 4.9, Proposition 2.1 and the mesh regularity (2.1), there exists $C>0$ depending only on $\Gamma, \operatorname{reg}(\mathcal{T})$ and the Lipschitz constant of $f_{b}^{\prime}$ such that

$$
\left\|g_{\mathfrak{M}}\left(t^{n+1}, \cdot\right)-g_{\mathfrak{M}}\left(t^{n}, \cdot\right)\right\|_{L^{2}(\Omega)}^{2} \leq 2 C_{12^{2}}^{2} \operatorname{size}(\mathcal{T})^{2}\left\|\nabla h^{n}\right\|_{H^{1}(\Omega)}^{2}+C \operatorname{size}(\mathcal{T})^{2} \Delta t \int_{t^{n}}^{t^{n+1}}\left\|\partial_{t} c\right\|_{H^{2}(\Omega)}^{2} .
$$

Moreover, there exists $M_{f_{b}}$ depending only on the Lipschitz constants of the derivatives of $f_{b}$ (up to 3) such that

$$
\begin{aligned}
\left\|\nabla h^{n}\right\|_{H^{1}(\Omega)}^{2} \leq M_{f_{b}} \Delta t( & \left(\|\nabla c\|_{L^{\infty}\left(0, T ; L^{\infty}(\Omega)\right)}^{4}+\left\|D^{2} c\right\|_{L^{\infty}\left(0, T ; L^{\infty}(\Omega)\right)}^{2}\right) \int_{t^{n}}^{t^{n+1}}\left\|\partial_{t} c\right\|_{L^{2}(\Omega)}^{2} \\
& \left.+\|\nabla c\|_{L^{\infty}\left(0, T ; L^{\infty}(\Omega)\right)}^{2} \int_{t^{n}}^{t^{n+1}}\left\|\partial_{t} \nabla c\right\|_{L^{2}(\Omega)}^{2}+\int_{t^{n}}^{t^{n+1}}\left\|\partial_{t} c\right\|_{H^{2}(\Omega)}^{2}\right)
\end{aligned}
$$

Summing these inequalities for $n$ going from 0 to $n_{0}$ gives estimate (4.33).

Now we have to deal with the main difficulty of the discretization of nonlinear terms. In each case the reasoning is similar. Thus we begin by proving the following general result.

LEMmA 4.19. Let us consider a function $\phi \in \mathcal{C}^{2}\left(\mathbb{R}^{2}, \mathbb{R}\right)$ such that all the derivatives up to the second order are bounded. Then, there exists $C_{21}>0$ depending only on $\phi$ and on the mesh regularity $\operatorname{reg}(\mathcal{T})$, such that for any $a_{\mathcal{T}}, b_{\mathcal{T}} \in \mathbb{R}^{\mathcal{T}}$ the following estimates hold

$$
\left|\phi\left(a_{\mathcal{T}}, b_{\mathcal{T}}\right)\right|_{1, \mathcal{T}} \leq C_{21}\left(\left|a_{\mathcal{T}}\right|_{1, \mathcal{T}}+\left|b_{\mathcal{T}}\right|_{1, \mathcal{T}}\right) .
$$

Moreover for any $\widetilde{b}_{\mathcal{T}} \in \mathbb{R}^{\mathcal{T}}$, one has

$$
\begin{aligned}
& \left|\phi\left(a_{\mathcal{T}}, b_{\mathcal{T}}\right)-\phi\left(b_{\mathcal{T}}, b_{\mathcal{T}}\right)\right|_{1, \mathcal{T}}^{2} \\
& \quad \leq C_{21}\left(\left|a_{\mathcal{T}}-b_{\mathcal{T}}\right|_{1, \mathcal{T}}^{2}+\left|b_{\mathcal{T}}-\widetilde{b}_{\mathcal{T}}\right|_{1, \mathcal{T}}^{2}+\operatorname{Lip}_{\mathcal{T}}\left(\widetilde{b}_{\mathcal{T}}\right)^{2}\left\|a_{\mathfrak{M}}-b_{\mathfrak{M}}\right\|_{L^{2}(\Omega)}^{2}\right)
\end{aligned}
$$

where $\operatorname{Lip}_{\mathcal{T}}\left(\widetilde{b}_{\mathcal{T}}\right)=\sup _{\sigma \in \mathcal{E}}\left|\frac{\widetilde{b}_{\mathcal{K}}-\widetilde{b}_{\mathcal{L}}}{d_{\mathcal{K}, \mathcal{L}}}\right|$.

Furthermore for any $a_{\partial \mathfrak{M}}, b_{\partial \mathfrak{M}} \in \mathbb{R}^{\partial \mathfrak{M}}$ we have

$$
\left\|\phi\left(a_{\partial \mathfrak{M}}, b_{\partial \mathfrak{M}}\right)-\phi\left(b_{\partial \mathfrak{M}}, b_{\partial \mathfrak{M}}\right)\right\|_{L^{2}(\Gamma)} \leq C_{21}\left\|a_{\partial \mathfrak{M}}-b_{\partial \mathfrak{M}}\right\|_{L^{2}(\Gamma)} .
$$

Proof. We first give the proof of estimate (4.35). Thanks to the definition of the discrete $H^{1}$-seminorm we have

$$
\left|\phi\left(a_{\mathcal{T}}, b_{\mathcal{T}}\right)-\phi\left(b_{\mathcal{T}}, b_{\mathcal{T}}\right)\right|_{1, \mathcal{T}}^{2}=\sum_{\sigma=\mathcal{K} \mid \mathcal{L} \in \mathcal{E}_{i n t}} \frac{m_{\sigma}}{d_{\mathcal{K}, \mathcal{L}}}\left(\phi_{\sigma}\left(a_{\mathcal{T}}, b_{\mathcal{T}}\right)\right)^{2}+\sum_{\sigma=\mathcal{L} \in \mathcal{E}_{\text {ext }}} \frac{m_{\mathbf{e}_{\mathcal{L}}}}{d_{\mathcal{K}, \mathcal{L}}}\left(\phi_{\sigma}\left(a_{\mathcal{T}}, b_{\mathcal{T}}\right)\right)^{2},
$$


where for any $\sigma=\mathcal{K} \mid \mathcal{L} \in \mathcal{E}_{\text {int }}$ or $\sigma=\mathcal{L} \in \mathcal{E}_{\text {ext }} \cap \mathcal{E}_{\mathcal{K}}($ with $\mathcal{K} \in \mathfrak{M})$ we note

$$
\phi_{\sigma}\left(a_{\mathcal{T}}, b_{\mathcal{T}}\right)=\left[\phi\left(a_{\mathcal{K}}, b_{\mathcal{K}}\right)-\phi\left(a_{\mathcal{L}}, b_{\mathcal{L}}\right)\right]-\left[\phi\left(b_{\mathcal{K}}, b_{\mathcal{K}}\right)-\phi\left(b_{\mathcal{L}}, b_{\mathcal{L}}\right)\right] .
$$

One has

$$
\begin{aligned}
\phi\left(a_{\mathcal{K}}, b_{\mathcal{K}}\right)-\phi\left(a_{\mathcal{L}}, b_{\mathcal{L}}\right)=\int_{0}^{1}( & \left(a_{\mathcal{K}}-a_{\mathcal{L}}\right) \partial_{1} \phi\left(a_{\mathcal{L}}+s\left(a_{\mathcal{K}}-a_{\mathcal{L}}\right), b_{\mathcal{L}}+s\left(b_{\mathcal{K}}-b_{\mathcal{L}}\right)\right) \\
& \left.\quad+\left(b_{\mathcal{K}}-b_{\mathcal{L}}\right) \partial_{2} \phi\left(a_{\mathcal{L}}+s\left(a_{\mathcal{K}}-a_{\mathcal{L}}\right), b_{\mathcal{L}}+s\left(b_{\mathcal{K}}-b_{\mathcal{L}}\right)\right)\right) \mathrm{d} s
\end{aligned}
$$

and

$$
\begin{aligned}
\phi\left(b_{\mathcal{K}}, b_{\mathcal{K}}\right)-\phi\left(b_{\mathcal{L}}, b_{\mathcal{L}}\right)=\int_{0}^{1}\left(\left(b_{\mathcal{K}}-b_{\mathcal{L}}\right) \partial_{1} \phi\left(b_{\mathcal{L}}+s\left(b_{\mathcal{K}}-b_{\mathcal{L}}\right), b_{\mathcal{L}}+s\left(b_{\mathcal{K}}-b_{\mathcal{L}}\right)\right)\right. \\
\left.\quad+\left(b_{\mathcal{K}}-b_{\mathcal{L}}\right) \partial_{2} \phi\left(b_{\mathcal{L}}+s\left(b_{\mathcal{K}}-b_{\mathcal{L}}\right), b_{\mathcal{L}}+s\left(b_{\mathcal{K}}-b_{\mathcal{L}}\right)\right)\right) \mathrm{d} s
\end{aligned}
$$

Then for any $\sigma \in \mathcal{E}$ the term $\phi_{\sigma}\left(a_{\mathcal{T}}, b_{\mathcal{T}}\right)$ can be written as follows

$$
\phi_{\sigma}\left(a_{\mathcal{T}}, b_{\mathcal{T}}\right)=\phi_{\sigma}^{1}\left(a_{\mathcal{T}}, b_{\mathcal{T}}\right)+\phi_{\sigma}^{2}\left(a_{\mathcal{T}}, b_{\mathcal{T}}\right),
$$

with

$$
\begin{aligned}
\phi_{\sigma}^{1}\left(a_{\mathcal{T}}, b_{\mathcal{T}}\right) & =\int_{0}^{1}\left(b_{\mathcal{K}}-b_{\mathcal{L}}\right) \\
\times & \left(\partial_{2} \phi\left(a_{\mathcal{L}}+s\left(a_{\mathcal{K}}-a_{\mathcal{L}}\right), b_{\mathcal{L}}+s\left(b_{\mathcal{K}}-b_{\mathcal{L}}\right)\right)-\partial_{2} \phi\left(b_{\mathcal{L}}+s\left(b_{\mathcal{K}}-b_{\mathcal{L}}\right), b_{\mathcal{L}}+s\left(b_{\mathcal{K}}-b_{\mathcal{L}}\right)\right)\right. \\
& \left.+\partial_{1} \phi\left(a_{\mathcal{L}}+s\left(a_{\mathcal{K}}-a_{\mathcal{L}}\right), b_{\mathcal{L}}+s\left(b_{\mathcal{K}}-b_{\mathcal{L}}\right)\right)-\partial_{1} \phi\left(b_{\mathcal{L}}+s\left(b_{\mathcal{K}}-b_{\mathcal{L}}\right), b_{\mathcal{L}}+s\left(b_{\mathcal{K}}-b_{\mathcal{L}}\right)\right)\right) \mathrm{d} s
\end{aligned}
$$

and

$$
\phi_{\sigma}^{2}\left(a_{\mathcal{T}}, b_{\mathcal{T}}\right)=\int_{0}^{1}\left(\left(a_{\mathcal{K}}-a_{\mathcal{L}}\right)-\left(b_{\mathcal{K}}-b_{\mathcal{L}}\right)\right) \partial_{1} \phi\left(a_{\mathcal{L}}+s\left(a_{\mathcal{K}}-a_{\mathcal{L}}\right), b_{\mathcal{L}}+s\left(b_{\mathcal{K}}-b_{\mathcal{L}}\right)\right) \mathrm{d} s
$$

For the term $\phi_{\sigma}^{2}\left(a_{\mathcal{T}}, b_{\mathcal{T}}\right)$, since the function $\partial_{1} \phi$ is bounded we obtain

$$
\left|\phi_{\sigma}^{2}\left(a_{\mathcal{T}}, b_{\mathcal{T}}\right)\right|_{1, \mathcal{T}} \leq\left\|\partial_{1} \phi\right\|_{L^{\infty}}\left|a_{\mathcal{T}}-b_{\mathcal{T}}\right|_{1, \mathcal{T}} .
$$

As regards the term $\phi_{\sigma}^{1}\left(a_{\mathcal{T}}, b_{\mathcal{T}}\right)$, we write

$$
\phi_{\sigma}^{1}\left(a_{\mathcal{T}}, b_{\mathcal{T}}\right)=\phi_{\sigma}^{1,1}\left(a_{\mathcal{T}}, b_{\mathcal{T}}\right)+\phi_{\sigma}^{1,2}\left(a_{\mathcal{T}}, b_{\mathcal{T}}\right),
$$

with

$$
\begin{aligned}
& \phi_{\sigma}^{1,1}\left(a_{\mathcal{T}}, b_{\mathcal{T}}\right)=\int_{0}^{1}\left(\left(b_{\mathcal{K}}-\widetilde{b}_{\mathcal{K}}\right)-\left(b_{\mathcal{L}}-\widetilde{b}_{\mathcal{L}}\right)\right) \\
& \quad \times\left(\partial_{2} \phi\left(a_{\mathcal{L}}+s\left(a_{\mathcal{K}}-a_{\mathcal{L}}\right), b_{\mathcal{L}}+s\left(b_{\mathcal{K}}-b_{\mathcal{L}}\right)\right)-\partial_{2} \phi\left(b_{\mathcal{L}}+s\left(b_{\mathcal{K}}-b_{\mathcal{L}}\right), b_{\mathcal{L}}+s\left(b_{\mathcal{K}}-b_{\mathcal{L}}\right)\right)\right. \\
& \left.\quad+\partial_{1} \phi\left(a_{\mathcal{L}}+s\left(a_{\mathcal{K}}-a_{\mathcal{L}}\right), b_{\mathcal{L}}+s\left(b_{\mathcal{K}}-b_{\mathcal{L}}\right)\right)-\partial_{1} \phi\left(b_{\mathcal{L}}+s\left(b_{\mathcal{K}}-b_{\mathcal{L}}\right), b_{\mathcal{L}}+s\left(b_{\mathcal{K}}-b_{\mathcal{L}}\right)\right)\right) \mathrm{d} s
\end{aligned}
$$


and

$$
\begin{aligned}
\phi_{\sigma}^{1,2}\left(a_{\mathcal{T}}, b_{\mathcal{T}}\right) & =\int_{0}^{1}\left(\widetilde{b}_{\mathcal{K}}-\widetilde{b}_{\mathcal{L}}\right) \\
\times & \left(\partial_{2} \phi\left(a_{\mathcal{L}}+s\left(a_{\mathcal{K}}-a_{\mathcal{L}}\right), b_{\mathcal{L}}+s\left(b_{\mathcal{K}}-b_{\mathcal{L}}\right)\right)-\partial_{2} \phi\left(b_{\mathcal{L}}+s\left(b_{\mathcal{K}}-b_{\mathcal{L}}\right), b_{\mathcal{L}}+s\left(b_{\mathcal{K}}-b_{\mathcal{L}}\right)\right)\right. \\
\quad & \left.+\partial_{1} \phi\left(a_{\mathcal{L}}+s\left(a_{\mathcal{K}}-a_{\mathcal{L}}\right), b_{\mathcal{L}}+s\left(b_{\mathcal{K}}-b_{\mathcal{L}}\right)\right)-\partial_{1} \phi\left(b_{\mathcal{L}}+s\left(b_{\mathcal{K}}-b_{\mathcal{L}}\right), b_{\mathcal{L}}+s\left(b_{\mathcal{K}}-b_{\mathcal{L}}\right)\right)\right) \mathrm{d} s .
\end{aligned}
$$

Since the functions $\partial_{1} \phi$ and $\partial_{2} \phi$, are bounded we get

$$
\left|\phi_{\sigma}^{1,1}\left(a_{\mathcal{T}}, b_{\mathcal{T}}\right)\right|_{1, \mathcal{T}} \leq \max \left(\left\|\partial_{1} \phi\right\|_{L^{\infty}},\left\|\partial_{2} \phi\right\|_{L^{\infty}}\right)\left|b_{\mathcal{T}}-\widetilde{b}_{\mathcal{T}}\right|_{1, \mathcal{T}} .
$$

Finally we have to deal with the term $\phi_{\sigma}^{1,2}\left(a_{\mathcal{T}}, b_{\mathcal{T}}\right)$. For any $i=1,2$, since $\partial_{1, i} \phi$ is bounded we have

$$
\begin{aligned}
\left|\partial_{i} \phi\left(a_{\mathcal{L}}+s\left(a_{\mathcal{K}}-a_{\mathcal{L}}\right), b_{\mathcal{L}}+s\left(b_{\mathcal{K}}-b_{\mathcal{L}}\right)\right)-\partial_{i} \phi\left(b_{\mathcal{L}}+s\left(b_{\mathcal{K}}-b_{\mathcal{L}}\right), b_{\mathcal{L}}+s\left(b_{\mathcal{K}}-b_{\mathcal{L}}\right)\right)\right| \\
\leq\left\|\partial_{1, i} \phi\right\|_{L^{\infty}}\left|(1-s)\left(a_{\mathcal{L}}-b_{\mathcal{L}}\right)+s\left(a_{\mathcal{K}}-b_{\mathcal{K}}\right)\right|
\end{aligned}
$$

and so for any $\sigma \in \mathcal{E}$, we obtain

$$
\left(\frac{\phi_{\sigma}^{1,2}\left(a_{\mathcal{T}}, b_{\mathcal{T}}\right)}{d_{\mathcal{K}, \mathcal{L}}}\right)^{2} \leq 2\left(\max \left(\left\|\partial_{1,1} \phi\right\|_{L^{\infty}},\left\|\partial_{1,2} \phi\right\|_{L^{\infty}}\right)\right)^{2}\left|\frac{\widetilde{b}_{\mathcal{K}}-\widetilde{b}_{\mathcal{L}}}{d_{\mathcal{K}, \mathcal{L}}}\right|^{2}\left(\left|a_{\mathcal{K}}-b_{\mathcal{K}}\right|^{2}+\left|a_{\mathcal{L}}-b_{\mathcal{L}}\right|^{2}\right)
$$

Since $d_{\mathcal{K}, \mathcal{L}} \leq \operatorname{reg}(\mathcal{T}) d\left(x_{\mathcal{K}}, \sigma\right)$ for any $\mathcal{K} \in \mathfrak{M}$, for any $\sigma \in \mathcal{E}_{\mathcal{K}}$ (see definition (2.1)), there exists $C(\operatorname{reg}(\mathcal{T}))$ such that

$$
\left|\phi_{\sigma}^{1,2}\left(a_{\mathcal{T}}, b_{\mathcal{T}}\right)\right|_{1, \mathcal{T}} \leq C(\operatorname{reg}(\mathcal{T})) \max \left(\left\|\partial_{1,1} \phi\right\|_{L^{\infty}},\left\|\partial_{1,2} \phi\right\|_{L^{\infty}}\right) \operatorname{Lip}_{\mathcal{T}}\left(\widetilde{b}_{\mathcal{T}}\right)\left\|a_{\mathfrak{M}}-b_{\mathfrak{M}}\right\|_{L^{2}(\Omega)},
$$

and estimate (4.35) yields.

Owing to (4.37) we also obtain estimate (4.34) and a similar reasoning gives estimate (4.36). . With this result at hand we can now bounded the terms $T_{f_{b}}$ (Proposition 4.20) and $T_{f_{s}}$ (Proposition 4.21).

Proposition 4.20. For any $n_{0} \in \llbracket 0, N \rrbracket$, there exists $C_{22}>0$ (depending on $f_{b}$ but not on $\Delta t$ and $\operatorname{size}(\mathcal{T})$ ) such that

$$
\begin{aligned}
\sum_{n=0}^{n_{0}} \Delta t & \left(\left|f_{b}^{\prime}\left(\mathbb{P}_{\mathcal{T}}^{c}\left(c\left(t^{n+1}\right)\right)\right)-f_{b}^{\prime}\left(c_{\mathcal{T}}^{n+1}\right)\right|_{1, \mathcal{T}}^{2}+\left|d^{f_{b}}\left(c_{\mathcal{T}}^{n+1}, c_{\mathcal{T}}^{n+1}\right)-d^{f_{b}}\left(c_{\mathcal{T}}^{n}, c_{\mathcal{T}}^{n+1}\right)\right|_{1, \mathcal{T}}^{2}\right) \\
\leq & C_{22}\left(1+\|\nabla c\|_{L^{\infty}\left(0, T ; L^{\infty}(\Omega)\right)}^{2}\right)\left(\sum_{n=1}^{n_{0}+1} \Delta t\left|\dot{e}_{\mathcal{T}}^{c, n}\right|_{1, \mathcal{T}}^{2}+\sum_{n=0}^{n_{0}} \Delta t\left|\dot{e}_{\mathcal{T}}^{c, n+1}-\dot{e}_{\mathcal{T}}^{c, n}\right|_{1, \mathcal{T}}^{2}\right) \\
& +C_{22} \operatorname{size}(\mathcal{T})^{2}\|c\|_{L^{\infty}\left(0, T ; H_{\Gamma}^{2}(\Omega)\right)}^{2} \\
& +C_{22}\|\nabla c\|_{L^{\infty}\left(0, T ; L^{\infty}(\Omega)\right)}^{2}\left(1+\left\|c^{0}\right\|_{H_{\Gamma}^{2}(\Omega)}^{2}+\|c\|_{L^{\infty}\left(0, T ; H_{\Gamma}^{2}(\Omega)\right)}^{2}\right) \operatorname{size}(\mathcal{T})^{2} \\
& +C_{22}\left(1+\|\nabla c\|_{L^{\infty}\left(0, T ; L^{\infty}(\Omega)\right)}^{2}\right)\left\|\partial_{t} \nabla c\right\|_{L^{2}\left(0, T ; L^{\infty}(\Omega)\right)}^{2} \Delta t^{2} \\
& +C_{22}\left(1+\|\nabla c\|_{L^{\infty}\left(0, T ; L^{\infty}(\Omega)\right)}^{2}\right)\left\|\partial_{t} c\right\|_{L^{2}\left(0, T ; H_{\Gamma}^{2}(\Omega)\right)}^{2} \Delta t^{2} \operatorname{size}(\mathcal{T})^{2}
\end{aligned}
$$


Proof. We will apply Lemma 4.19 twice with a good choice of the functions which intervene. First, we apply the lemma to the function $\phi$ defined by $\phi(x, y)=f_{b}^{\prime}(x)$ for any $x, y \in \mathbb{R}$. Then we choose $a_{\mathcal{T}}=c_{\mathcal{T}}^{n+1}$ and $b_{\mathcal{T}}=\widetilde{b}_{\mathcal{T}}=\mathbb{P}_{\mathcal{T}}^{c}\left(c\left(t^{n+1}\right)\right)$, and so thanks to (4.35) we obtain

$$
\begin{aligned}
\mid f_{b}^{\prime}\left(\mathbb{P}_{\mathcal{T}}^{c}\left(c\left(t^{n+1}\right)\right)\right)-f_{b}^{\prime}\left(c_{\mathcal{T}}^{n+1}\right) & \left.\right|_{1, \mathcal{T}} ^{2} \\
& \leq C_{21}\left(\left|e_{\mathcal{T}}^{c, n+1}\right|_{1, \mathcal{T}}^{2}+\operatorname{Lip}_{\mathcal{T}}\left(\mathbb{P}_{\mathcal{T}}^{c}\left(c\left(t^{n+1}\right)\right)\right)^{2}\left\|e_{\mathfrak{M}}^{c, n+1}\right\|_{L^{2}(\Omega)}^{2}\right)
\end{aligned}
$$

Now we apply Lemma 4.19 to the function $\phi=d^{f_{b}}$ with $a_{\mathcal{T}}=c_{\mathcal{T}}^{n}, b_{\mathcal{T}}=c_{\mathcal{T}}^{n+1}$ and $\widetilde{b}_{\mathcal{T}}=$ $\mathbb{P}_{\mathcal{T}}^{c}\left(c\left(t^{n+1}\right)\right)$. Inequality (4.35) implies

$$
\begin{aligned}
& \left|d^{f_{b}}\left(c_{\mathcal{T}}^{n+1}, c_{\mathcal{T}}^{n+1}\right)-d^{f_{b}}\left(c_{\mathcal{T}}^{n}, c_{\mathcal{T}}^{n+1}\right)\right|_{1, \mathcal{T}}^{2} \\
& \quad \leq C_{21}\left(\left|c_{\mathcal{T}}^{n+1}-c_{\mathcal{T}}^{n}\right|_{1, \mathcal{T}}^{2}+\left|e_{\mathcal{\mathcal { T }}}^{c, n+1}\right|_{1, \mathcal{T}}^{2}+\operatorname{Lip}_{\mathcal{\mathcal { T }}}\left(\mathbb{P}_{\mathcal{T}}^{c}\left(c\left(t^{n+1}\right)\right)\right)^{2}\left\|c_{\mathfrak{M}}^{n+1}-c_{\mathfrak{M}}^{n}\right\|_{L^{2}(\Omega)}^{2}\right)
\end{aligned}
$$

Thanks to the mean-value theorem, we have

$$
\operatorname{Lip}_{\mathcal{T}}\left(\mathbb{P}_{\mathcal{T}}^{c}\left(c\left(t^{n+1}\right)\right)\right) \leq\|\nabla c\|_{L^{\infty}\left(0, T ; L^{\infty}(\Omega)\right)} .
$$

The definition of the elliptic projection and the volume conservation at the discrete level (3.3) imply $m_{\underline{\Omega}}\left(\dot{e}_{\mathfrak{M}}^{c, n}\right)=m_{\underline{\Omega}}\left(\dot{e}_{\mathfrak{M}}^{c, 0}\right)$, thus thanks to the Poincaré inequality (2.3), the bound on the initial data (Proposition 4.15), and equation (4.23) we deduce

$$
\begin{aligned}
\left\|\dot{e}_{\mathfrak{M}}^{c, n}\right\|_{L^{2}(\Omega)}^{2} \leq & 3 C_{4}^{2}\left|\dot{e}_{\mathcal{T}}^{c, n}\right|_{1, \mathcal{T}}^{2}+\frac{3 C_{16}^{2}}{C_{1}^{2}}|\Omega| \operatorname{size}(\mathcal{T})^{2}\left\|c^{0}\right\|_{H_{\Gamma}^{2}(\Omega)}^{2} \\
& +3 C_{5}^{2}\left(M_{1}+\left(C_{15}+1\right)\|c\|_{L^{\infty}\left(0, T ; H_{\Gamma}^{2}(\Omega)\right)}\right)^{2} \operatorname{size}(\mathcal{T})^{2}
\end{aligned}
$$

Moreover Lemma 4.9 and Proposition 4.11 give

$$
\begin{aligned}
\left\|\ddot{e}_{\mathfrak{M}}^{c, n}\right\|_{L^{2}(\Omega)} & \leq\left(C_{12}+C_{15}\right) \operatorname{size}(\mathcal{T})\left\|c\left(t^{n}, \cdot\right)\right\|_{H_{\Gamma}^{2}(\Omega)}, \\
\left|\ddot{e}_{\mathfrak{M}}^{c, n}\right|_{1, \mathcal{T}} & \leq C_{15} \operatorname{size}(\mathcal{T})\left\|c\left(t^{n}, \cdot\right)\right\|_{H_{\Gamma}^{2}(\Omega)} .
\end{aligned}
$$

Thus, gathering estimates (4.42) and (4.43) there exists $C_{23}>0$ such that for any $n \in \llbracket 0, N \rrbracket$,

$$
\left\|e_{\mathfrak{M}}^{c, n}\right\|_{L^{2}(\Omega)}^{2} \leq C_{23}\left|\dot{e}_{\mathcal{T}}^{c, n}\right|_{1, \mathcal{T}}^{2}+C_{23} \operatorname{size}(\mathcal{T})^{2}\left(1+\left\|c^{0}\right\|_{H_{\Gamma}^{2}(\Omega)}^{2}+\|c\|_{L^{\infty}\left(0, T ; H_{\Gamma}^{2}(\Omega)\right)}^{2}\right) .
$$

Now, we have to deal with the terms on the right-hand side of (4.40). First, we write

$$
\left|c_{\mathcal{T}}^{n+1}-c_{\mathcal{T}}^{n}\right|_{1, \mathcal{T}} \leq\left|\dot{e}_{\mathcal{T}}^{c, n+1}-\dot{e}_{\mathcal{T}}^{c, n}\right|_{1, \mathcal{T}}+\left|\ddot{e}_{\mathcal{T}}^{c, n+1}-\ddot{e}_{\mathcal{T}}^{c, n}\right|_{1, \mathcal{T}}+\left|\mathbb{P}_{\mathcal{T}}^{c} c\left(t^{n+1}\right)-\mathbb{P}_{\mathcal{T}}^{c} c\left(t^{n}\right)\right|_{1, \mathcal{T}}
$$

Since the projections $\mathbb{P}_{\mathcal{T}}^{\mathrm{ell}, \mathrm{D}}$ and $\mathbb{P}_{\mathcal{T}}^{c}$ are linear we can apply Proposition 4.11 that implies

$$
\left|\ddot{e}_{\mathcal{T}}^{c, n+1}-\ddot{e}_{\mathcal{T}}^{c, n}\right|_{1, \mathcal{T}}^{2} \leq C_{15}^{2} \Delta t \operatorname{size}(\mathcal{T})^{2} \int_{t^{n}}^{t^{n+1}}\left\|\partial_{t} c(t, \cdot)\right\|_{H_{\Gamma}^{2}(\Omega)}^{2} \mathrm{~d} t
$$

Moreover, thanks to Proposition 2.3 there exists $C>0$ depending only on $\Omega$ and $\operatorname{reg}(\mathcal{T})$ such 
that

$$
\begin{aligned}
& \left|\mathbb{P}_{\mathcal{T}}^{c} c\left(t^{n+1}\right)-\mathbb{P}_{\mathcal{T}}^{c} c\left(t^{n}\right)\right|_{1, \mathcal{T}}^{2} \\
& \leq \Delta t \sum_{\sigma=\mathcal{K} \mid \mathcal{L} \in \mathcal{E}_{i n t}} m_{\sigma} d_{\mathcal{K}, \mathcal{L}} \int_{t^{n}}^{t^{n+1}}\left|\int_{0}^{1} \partial_{t} \nabla c\left(t, x_{\mathcal{L}}+s\left(x_{\mathcal{K}}-x_{\mathcal{L}}\right)\right) \cdot \overrightarrow{\mathbf{n}}_{\mathcal{K} \mathcal{L}} \mathrm{d} s\right|^{2} \mathrm{~d} t \\
& \quad+\Delta t \sum_{\sigma=\mathcal{L} \in \mathcal{E}_{\text {ext }}} m_{\mathbf{e}_{\mathcal{L}}} d_{\mathcal{K}, \mathcal{L}} \int_{t^{n}}^{t^{n+1}}\left|\frac{d\left(x_{\mathcal{K}}, x_{\mathcal{L}}\right)}{d_{\mathcal{K}, \mathcal{L}}} \int_{0}^{1} \partial_{t} \nabla c\left(t, x_{\mathcal{L}}+s\left(x_{\mathcal{K}}-x_{\mathcal{L}}\right)\right) \cdot \overrightarrow{\mathbf{n}}_{\mathcal{K} \mathcal{L}} \mathrm{d} s\right|^{2} \mathrm{~d} t \\
& \quad \leq C \Delta t \int_{t^{n}}^{t^{n+1}}\left\|\partial_{t} \nabla c(t, \cdot)\right\|_{L^{\infty}(\Omega)}^{2} \mathrm{~d} t .
\end{aligned}
$$

Therefore we have

$$
\begin{aligned}
\left|c_{\mathcal{T}}^{n+1}-c_{\mathcal{T}}^{n}\right|_{1, \mathcal{T}}^{2} \leq & 3\left|\dot{e}_{\mathcal{T}}^{c, n+1}-\dot{e}_{\mathcal{T}}^{c, n}\right|_{1, \mathcal{T}}^{2}+3 C_{15}^{2} \Delta t \operatorname{size}(\mathcal{T})^{2} \int_{t^{n}}^{t^{n+1}}\left\|\partial_{t} c(t, \cdot)\right\|_{H_{\Gamma}^{2}(\Omega)}^{2} \\
& +3 C \Delta t \int_{t^{n}}^{t^{n+1}}\left\|\partial_{t} \nabla c(t, \cdot)\right\|_{L^{\infty}(\Omega)}^{2} \mathrm{~d} t
\end{aligned}
$$

Finally, the discrete conservation of the volume (3.3) implies $m_{\underline{\Omega}}\left(c_{\mathfrak{M}}^{n+1}-c_{\mathfrak{M}}^{n}\right)=0$ and so the Poincaré inequality (2.3) and the bound on the discrete solutions in Proposition 4.5 give

$$
\left\|c_{\mathfrak{M}}^{n+1}-c_{\mathfrak{M}}^{n}\right\|_{L^{2}(\Omega)} \leq C_{4}\left|c_{\mathcal{T}}^{n+1}-c_{\mathcal{T}}^{n}\right|_{1, \mathcal{T}}+2 C_{5} M_{1} \operatorname{size}(\mathcal{T})
$$

Summing (4.39) and (4.40), gathering estimates (4.41), (4.43), (4.44), (4.45) and (4.46) and summing the resulting inequality for $n$ going from 0 to $n_{0}$ conclude the proof.

Proposition 4.21. For any $n_{0} \in \llbracket 0, N \rrbracket$, there exists $C_{24}>0$ independent of $\Delta t$ and $\operatorname{size}(\mathcal{T})$ (but depending on $f_{s}$ ) such that

$$
\begin{aligned}
& \sum_{n=0}^{n_{0}} \Delta t\left\|f_{s}^{\prime}\left(\mathbb{P}_{\partial \mathfrak{M}}^{c}\left(c\left(t^{n+1}\right)\right)\right)-d^{f_{s}}\left(c_{\partial \mathfrak{M}}^{n}, c_{\partial \mathfrak{M}}^{n+1}\right)\right\|_{L^{2}(\Gamma)} \leq C_{24} \sum_{n=0}^{n_{0}} \Delta t\left|\dot{e}_{\mathcal{T}}^{c, n+1}\right|_{1, \mathcal{T}}^{2} \\
& \quad+C_{24} \Delta t^{2}\left(\sum_{n=0}^{n_{0}} \Delta t\left\|\frac{\dot{e}_{\partial \mathfrak{M}}^{c, n+1}-\dot{e}_{\partial \mathfrak{M}}^{c, n}}{\Delta t}\right\|_{0, \partial \mathfrak{M}}^{2}+\operatorname{size}(\mathcal{T})^{2}\left\|\partial_{t} c\right\|_{L^{2}\left(0, T ; H_{\Gamma}^{2}(\Omega)\right)}^{2}+\left\|\partial_{t} c_{\Gamma \Gamma}\right\|_{L^{2}\left(0, T ; H^{1}(\Gamma)\right)}^{2}\right) \\
& \quad+C_{24} \operatorname{size}(\mathcal{T})^{2}\left(\left\|c^{0}\right\|_{H_{\Gamma}^{2}(\Omega)}^{2}+\|c\|_{L^{\infty}\left(0, T ; H_{\Gamma}^{2}(\Omega)\right)}^{2}\right) .
\end{aligned}
$$

Proof. Applying inequality (4.36) to the function $\phi=f_{s}^{\prime}$ at first, and then, to $\phi=d^{f_{s}}$ give

$$
\left\|f_{s}^{\prime}\left(\mathbb{P}_{\partial \mathfrak{M}}^{c}\left(c\left(t^{n+1}\right)\right)\right)-d^{f_{s}}\left(c_{\partial \mathfrak{M}}^{n}, c_{\partial \mathfrak{M}}^{n+1}\right)\right\|_{L^{2}(\Gamma)} \leq C_{21}\left(\left\|e_{\partial \mathfrak{M}}^{c, n+1}\right\|_{L^{2}(\Gamma)}+\left\|c_{\partial \mathfrak{M}}^{n+1}-c_{\partial \mathfrak{M}}^{n}\right\|_{L^{2}(\Gamma)}\right) .
$$

Owing to Lemma 2.10 and estimate (4.42) we have

$$
\left\|\dot{e}_{\partial \mathfrak{M}}^{c, n+1}\right\|_{L^{2}(\Gamma)}^{2} \leq 2 C_{8}^{2}\left(\left(1+2 C_{2} C_{4}^{2}\right)\left|\dot{e}_{\mathcal{T}}^{c, n+1}\right|_{1, \mathcal{T}}^{2}+\frac{2 C_{2} C_{16}^{2}}{C_{1}}|\Omega| \operatorname{size}(\mathcal{T})^{2}\left\|c^{0}\right\|_{H_{\Gamma}^{2}(\Omega)}^{2}\right) .
$$

Moreover, thanks to Lemma 4.9 and Proposition 4.11 we obtain

$$
\left\|\ddot{e}_{\partial \mathfrak{M}}^{c, n+1}\right\|_{L^{2}(\Gamma)} \leq\left(C_{13}+C_{15}\right) \operatorname{size}(\mathcal{T})\left\|c\left(t^{n+1}, \cdot\right)\right\|_{H_{\Gamma}^{2}(\Omega)} .
$$


Finally we write

$\left\|c_{\partial \mathfrak{M}}^{n+1}-c_{\partial \mathfrak{M}}^{n}\right\|_{L^{2}(\Gamma)} \leq\left\|\dot{e}_{\partial \mathfrak{M}}^{c, n+1}-\dot{e}_{\partial \mathfrak{M}}^{c, n}\right\|_{L^{2}(\Gamma)}+\left\|\ddot{e}_{\partial \mathfrak{M}}^{c, n+1}-\ddot{e}_{\partial \mathfrak{M}}^{c, n}\right\|_{L^{2}(\Gamma)}+\left\|\mathbb{P}_{\partial \mathfrak{M}}^{c} c\left(t^{n+1}\right)-\mathbb{P}_{\partial \mathfrak{M}}^{c} c\left(t^{n}\right)\right\|_{L^{2}(\Gamma)}$.

Then, the linearity of the projection $\mathbb{P}_{\partial \mathfrak{M}}^{\mathrm{ell}, \mathrm{D}}$ and $\mathbb{P}_{\partial \mathfrak{M}}^{c}$, Lemma 4.9 and Proposition 4.11 give

$$
\left\|\ddot{e}_{\partial \mathfrak{M}}^{c, n+1}-\ddot{e}_{\partial \mathfrak{M}}^{c, n}\right\|_{L^{2}(\Gamma)}^{2} \leq\left(C_{13}+C_{15}\right)^{2} \operatorname{size}(\mathcal{T})^{2} \Delta t \int_{t^{n}}^{t^{n+1}}\left\|\partial_{t} c(t, \cdot)\right\|_{H_{\Gamma}^{2}(\Omega)}^{2} \mathrm{~d} t
$$

and

$$
\left\|\mathbb{P}_{\partial \mathfrak{M}}^{c} c\left(t^{n+1}\right)-\mathbb{P}_{\partial \mathfrak{M}}^{c} c\left(t^{n}\right)\right\|_{L^{2}(\Gamma)}^{2} \leq 2\left(C_{13}^{2}+1\right) \Delta t \int_{t^{n}}^{t^{n+1}}\left\|\partial_{t} c_{\mid \Gamma}(t, \cdot)\right\|_{H^{1}(\Gamma)}^{2} \mathrm{~d} t .
$$

Thus gathering these estimates and summing the resulting inequality between 0 and $n_{0}$ conclude the proof.

4.3.3. End of the proof of Theorem 4.2. We are now able to apply the discrete Gronwall lemma and to conclude the proof. Gathering Propositions 4.15, 4.16, 4.17, 4.18, 4.20 and 4.21, we can estimate term (4.16) (see Proposition 4.14). Thus, there exists $C_{25}>0$ (depending on $c$ and $\mu$ ) independent of $\operatorname{size}(\mathcal{T})$ and $\Delta t$ such that for any $n_{0} \in \llbracket 0, N-1 \rrbracket$,

$$
\begin{aligned}
& \frac{1}{2} \sum_{n=0}^{n_{0}} \Delta t\left|\dot{e}_{\mathcal{T}}^{\mu, n+1}\right|_{1, \mathcal{T}}^{2}+\frac{1}{4}\left|\dot{e}_{\mathcal{T}}^{c, n_{0}+1}\right|_{1, \mathcal{T}}^{2}+\frac{1}{2}\left|\dot{e}_{\partial \mathfrak{M}}^{c, n_{0}+1}\right|_{1, \partial \mathfrak{M}}^{2}+\frac{1}{2} \sum_{n=0}^{n_{0}}\left|\dot{e}_{\mathcal{T}}^{c, n+1}-\dot{e}_{\mathcal{T}}^{c, n}\right|_{1, \mathcal{T}}^{2} \\
& +\frac{1}{2} \sum_{n=0}^{n_{0}} \Delta t\left\|\frac{\dot{e}_{\partial \mathfrak{M}}^{c, n+1}-\dot{e}_{\partial \mathfrak{M}}^{c, n}}{\Delta t}\right\|_{0, \partial \mathfrak{M}}^{2}+\frac{1}{2} \sum_{n=0}^{n_{0}}\left|\dot{e}_{\partial \mathfrak{M}}^{c, n+1}-\dot{e}_{\partial \mathfrak{M}}^{c, n}\right|_{1, \partial \mathfrak{M}}^{2} \\
& \leq C_{25}\left(\operatorname{size}(\mathcal{T})^{2}+\Delta t^{2}\right)+C_{25} \Delta t\left|\dot{e}_{\mathcal{T}}^{c, n_{0}+1}\right|_{1, \mathcal{T}}^{2}+\left(1+C_{25}\right) \sum_{n=0}^{n_{0}} \Delta t\left|\dot{e}_{\mathcal{T}}^{c, n}\right|_{1, \mathcal{T}}^{2} \\
& +C_{25} \Delta t \sum_{n=0}^{n_{0}}\left|\dot{e}_{\mathcal{T}}^{c, n+1}-\dot{e}_{\mathcal{T}}^{c, n}\right|_{1, \mathcal{T}}^{2}+C_{25} \Delta t^{2} \sum_{n=0}^{n_{0}} \Delta t\left\|\frac{\dot{e}_{\partial \mathfrak{M}}^{c, n+1}-\dot{e}_{\partial \mathfrak{M}}^{c, n}}{\Delta t}\right\|_{0, \partial \mathfrak{M}}^{2} .
\end{aligned}
$$

Thus by choosing $\Delta t \leq \min \left(\frac{1}{8 C_{25}}, \frac{1}{2 \sqrt{C_{25}}}\right)$ we deduce

$$
\frac{1}{8}\left|\dot{e}_{\mathcal{T}}^{c, n_{0}+1}\right|_{1, \mathcal{T}}^{2} \leq\left(1+C_{25}\right) \sum_{n=0}^{n_{0}} \Delta t\left|\dot{e}_{\mathcal{T}}^{c, n}\right|_{1, \mathcal{T}}^{2}+C_{25}\left(\operatorname{size}(\mathcal{T})^{2}+\Delta t^{2}\right)
$$

Thanks to the discrete Gronwall lemma associated with estimates (4.42) and (4.43), Proposition 4.15 on the initial data and Proposition 4.11 we conclude the proof of Theorem 4.2.

5. Numerical error estimate. In the section we present numerical error estimate which illustrate the previous result. Several qualitative numerical results which show the different behaviours of the scheme in function of the properties of the components and the wall are performed in [22].

The domain $\Omega$ is the unit circle and we consider a Delaunay triangular mesh for which for any $\mathcal{K} \in \mathfrak{M}$ the center $x_{\mathcal{K}}$ is the circumcenter of the triangle $\mathcal{K}$ (or $\underline{\mathcal{K}}$ if $\partial \mathcal{K} \cap \mathcal{E}_{\text {ext }} \neq \emptyset$ ) and for any $\mathcal{L} \in \partial \mathfrak{M}, y_{\mathcal{L}}$ is the middle of the chord $\mathbf{e}_{\mathcal{L}}$. Since we do not know non-trivial solution of problem (1.1), we compute a reference solution on a very fine mesh $\mathcal{T}_{\text {ref }}$ whose mesh size is $\operatorname{size}\left(\mathcal{T}_{\text {ref }}\right) \sim 0.01$ and a very small time step $\Delta t_{\text {ref }}=10^{-5}$. The initial concentration is given by

$$
c_{0}(x, y)=\frac{1}{2}(1+0.01(\sin (4 \pi x)+\sin (4 \pi y))),
$$




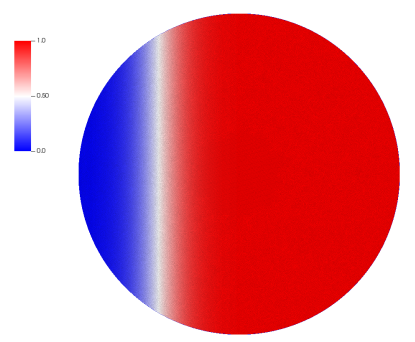

Fig. 3: Reference solution at time $T=0.5$

and the reference solution at final time $T=0.5$ is plotted in Fig. 3.

In equation (1.1) we choose the following parameters: the interface thickness $\varepsilon=0.5$, the mobility and the surface tension $\Gamma_{b}=\sigma_{b}=0.1$, the capillarity coefficient $\sigma_{s}=5$ and the relaxation coefficient $\Gamma_{s}=10$. As regards the non-linear potentials we set $f_{b}(c)=f_{s}(c)=$ $c^{2}(1-c)^{2}$ (see Fig. 1b) and we use the semi-implicit discretization.

To compare the approximate solution (computed on a coarse mesh) with the reference solution (computed on a fine mesh), we have to interpolate the reference solution on the coarse mesh. Then, at final time $T=0.5$, we plot the relative error between the interpolate of the reference solution $\mathcal{I}_{\mathcal{T}}\left(c_{\mathcal{T}_{\text {ref }}}^{N_{\text {ref }}}\right)$ and the approximate solution $c_{\mathcal{T}}^{N}$, namely $\frac{\left\|\mathcal{I}_{\mathcal{T}}\left(c_{\mathcal{T}_{\text {ref }}}^{N_{\text {ref }}}\right)-c_{\mathcal{T}}^{N}\right\|}{\left\|\mathcal{I}_{\mathcal{T}}\left(c_{\mathcal{T}_{\text {ref }}}^{N_{\text {ref }}}\right)\right\|}$, for the $L^{2}$-norm and the $H^{1}$-seminorm in $\underline{\Omega}$ and on $\partial \underline{\Omega}$ in two cases:

- when the time step tends to 0 and a fixed mesh size (see Fig. 4 and 5);

- when the mesh size tends to 0 and a fixed time step (see Fig. 6 and 7).

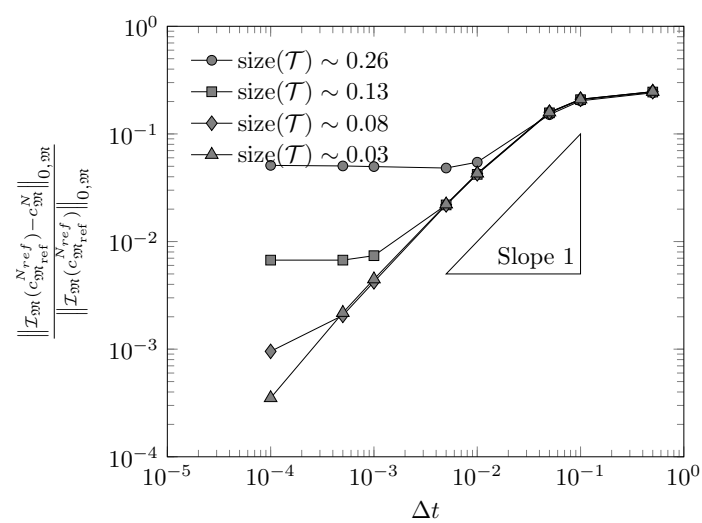

(a) $L^{2}$-error in $\Omega$

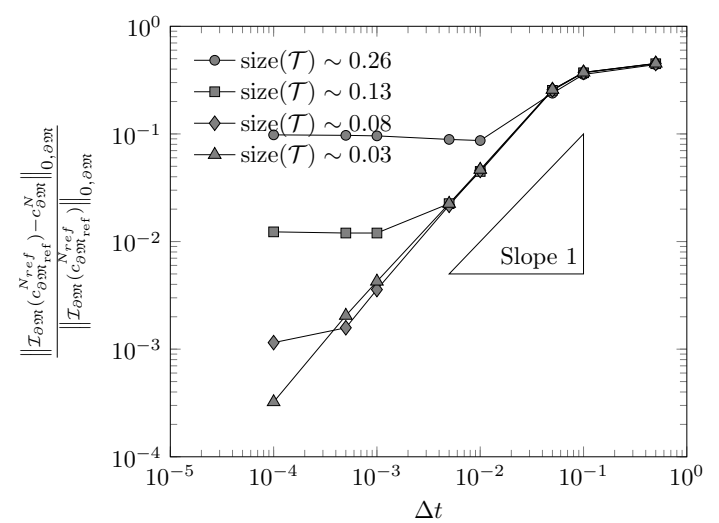

(b) $L^{2}$-error on $\Gamma$

Fig. 4: First-order convergence in time for the $L^{2}$-norms

First we observe that, as expected, we obtain a first-order convergence in time in each case (see Fig. 4 and 5).

For the space convergence we have several behaviours. Noting that when we study the Laplace problem with a finite-volume two-point flux approximation, from a computational point of view we observe a second-order convergence for the $L^{2}$-norm while at the theoretical 


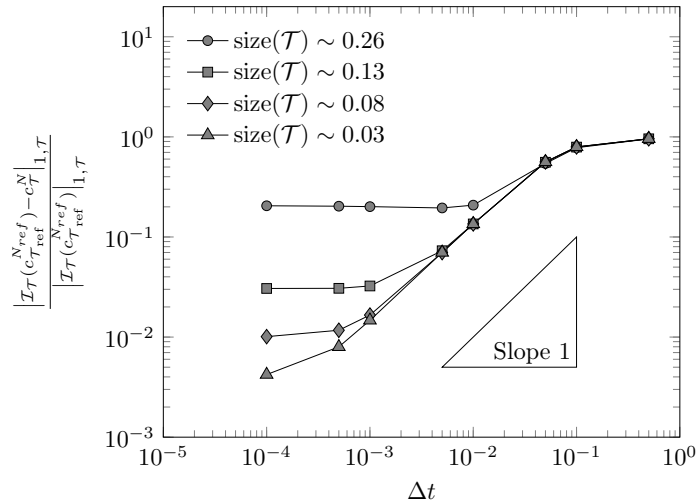

(a) $H^{1}$-error in $\Omega$

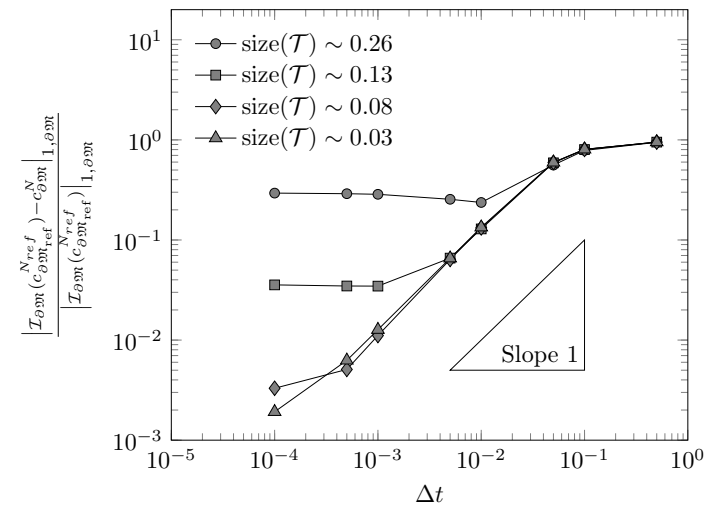

(b) $H^{1}$-error on $\Gamma$

Fig. 5: First-order convergence in time for the $H^{1}$-seminorms

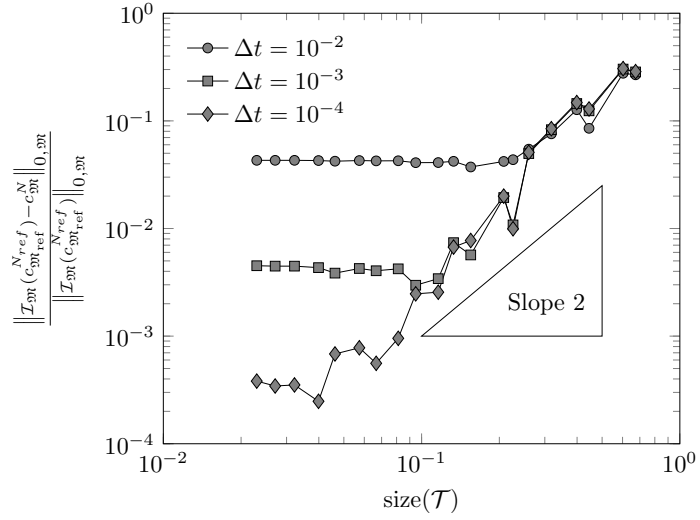

(a) $L^{2}$-error in $\Omega$

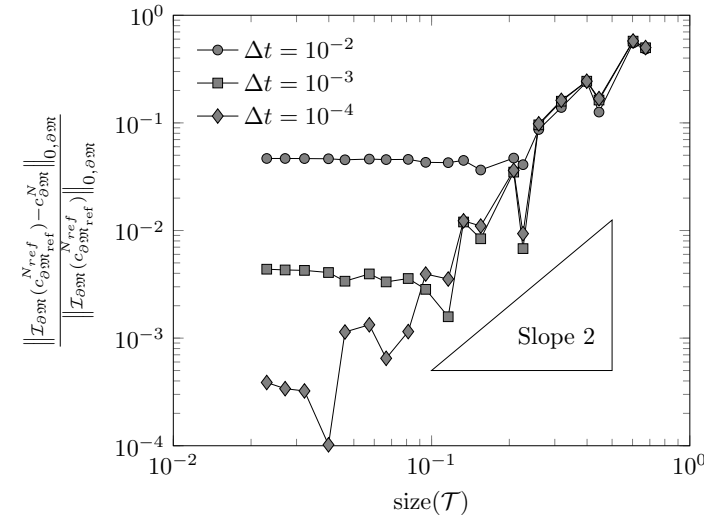

(b) $L^{2}$-error on $\Gamma$

Fig. 6: Second-order convergence in space for the $L^{2}$-norms

level, we are only able to prove the first-order convergence. A proof of this super-convergence phenomena is given in a recent work [4] for particular triangular meshes used in benchmarking. To our knowledge, the case of a general 2D triangular mesh, is still an open problem. We observe here the same super-convergence phenomena for the $L^{2}$-norms (see Fig. 6). As regards the $H^{1}(\Omega)$-seminorm (see Fig. 7a), although we observe a second-order convergence when the mesh size is coarse, we recover asymptotically the expected first-order convergence. However for the $H^{1}(\Gamma)$-seminorm (see Fig. $7 \mathrm{~b}$ ) we observe a second-order convergence instead of the first-order. We can assume that this super-convergence phenomena is due to the uniform geometry of the boundary mesh.

Appendix A. Proof of Proposition 4.11. For $f \in L^{2}(\Omega)$ and $g \in L^{2}(\Gamma)$, we consider 


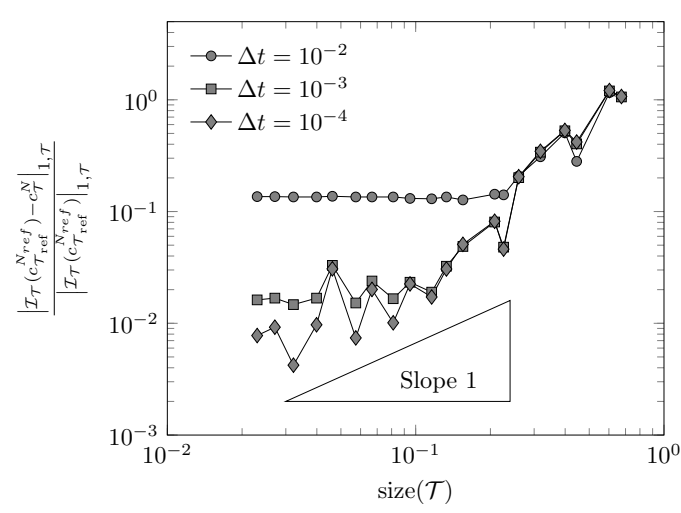

(a) $H^{1}$-error in $\Omega$

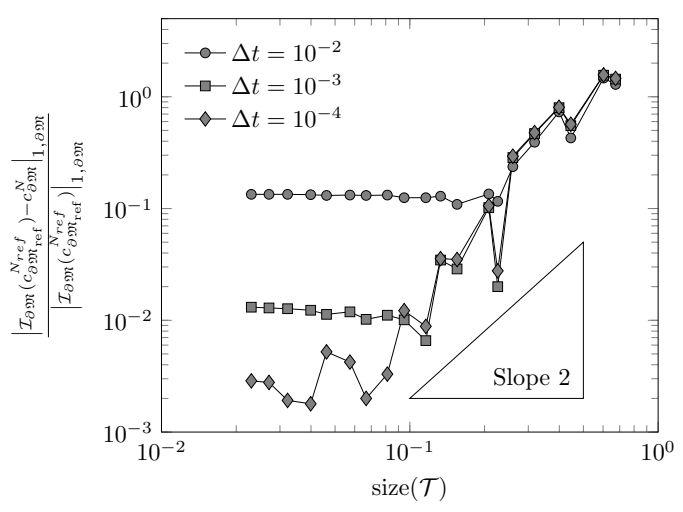

(b) $H^{1}$-error on $\Gamma$

Fig. 7: Convergence in space for the $H^{1}$-seminorms

the following problem: find $u: \Omega \rightarrow \mathbb{R}$ such that $\int_{\Omega} u=\alpha$ and

$$
\begin{array}{ll}
-\Delta u=f & \text { in } \Omega ; \\
-\Delta_{\Gamma} u_{\Gamma \Gamma}+\partial_{n} u=g & \text { on } \Gamma .
\end{array}
$$

By integrating equation (A.1a) on all interior control volumes $\mathcal{K} \in \mathfrak{M}$ and the boundary condition (A.1b) on all boundary control volumes $\mathcal{L} \in \partial \mathfrak{M}$, the two-point flux approximation of problem (A.1) writes as follows. Find $u_{\mathcal{T}} \in \mathbb{R}^{\tau}$ such that $\sum_{\mathcal{K} \in \mathfrak{M}} m_{\underline{\mathcal{K}}} u_{\mathcal{K}}=\alpha$ and

$$
\begin{aligned}
\sum_{\sigma \in \mathcal{E}_{\mathcal{K}}^{i n t}} m_{\sigma} \frac{u_{\mathcal{K}}-u_{\mathcal{L}}}{d_{\mathcal{K}, \mathcal{L}}}+\sum_{\sigma \in \mathcal{E}_{\mathcal{K}}^{\text {ext }}} m_{\mathbf{e}_{\mathcal{L}}} \frac{u_{\mathcal{K}}-u_{\mathcal{L}}}{d_{\mathcal{K}, \mathcal{L}}}=m_{\mathcal{K}} \mathbb{P}_{\mathcal{K}}^{m} f, \quad \forall \mathcal{K} \in \mathfrak{M} ; \\
\sum_{\mathbf{v} \in \mathcal{V}_{\mathcal{L}}} \frac{u_{\mathcal{L}}-u_{\mathcal{L}^{\prime}}}{d_{\mathcal{L}, \mathcal{L}^{\prime}}}+m_{\mathbf{e}_{\mathcal{L}}} \frac{u_{\mathcal{L}}-u_{\mathcal{K}}}{d_{\mathcal{K}, \mathcal{L}}}=m_{\mathcal{L}} \mathbb{P}_{\mathcal{L}}^{m} g, \quad \forall \mathcal{L} \in \partial \mathfrak{M} .
\end{aligned}
$$

We can prove that this problem admits a unique solution.

Because of the complex geometry of $\Omega$, it is possible to take into account some points $x \in \Omega^{c}$ in the proof of the error estimate. To ensure that all the quantities used in the proof of Theorem A.3 are well defined, we will use an extension in $\mathbb{R}^{2}$ of the function $u$. Since $u \in H^{2}(\Omega)$, there exists an extension $\widetilde{u} \in H^{2}\left(\mathbb{R}^{2}\right)$ (that we fix in the sequel) such that

$$
\widetilde{u}(x)=u(x), \quad \forall x \in \Omega \text { and }\|\widetilde{u}\|_{H^{2}\left(\mathbb{R}^{2}\right)} \leq C_{26}\|u\|_{H^{2}(\Omega)},
$$

with $C_{26}>0$ depending only on $\Omega$.

Proposition A.1. The tangential gradient of $u: \Gamma \rightarrow \mathbb{R}$ to the vertex $\mathbf{v}=\mathcal{L} \mid \mathcal{L}^{\prime}$ satisfies

$$
\left|\frac{u\left(x_{\mathcal{L}^{\prime}}\right)-u\left(x_{\mathcal{L}}\right)}{m_{\gamma_{\mathcal{L} \mathcal{L}^{\prime}}}}-\nabla_{\Gamma} u(\mathbf{v}) \cdot \overrightarrow{\boldsymbol{\tau}}_{\mathbf{v}, \mathcal{L}}(\mathbf{v})\right| \leq \int_{\gamma_{\mathcal{L} \mathcal{L}^{\prime}}}\left|(u \circ \varphi)^{\prime \prime}\left(\varphi^{-1}(x)\right)\right| \mathrm{d} \sigma(x),
$$

where $\varphi$ is an arc-length parametrization of the curve $\Gamma$ and $\overrightarrow{\boldsymbol{\tau}}_{\mathbf{v}, \mathcal{L}}(\mathbf{v})$ is the unit tangent vector to $\Gamma$ at point $\mathbf{v}=\mathcal{L} \mid \mathcal{L}^{\prime}$ going from $\mathcal{L}$ to $\mathcal{L}^{\prime}$. 
Proof. Let us consider the points $t_{\mathcal{L}}, t_{\mathcal{L}^{\prime}}, t_{\mathrm{v}} \in \mathbb{R}$ such that $x_{\mathcal{L}}=\varphi\left(t_{\mathcal{L}}\right), x_{\mathcal{L}^{\prime}}=\varphi\left(t_{\mathcal{L}^{\prime}}\right)$ and $\mathbf{v}=\varphi\left(t_{\mathbf{v}}\right)$, then the Taylor's formulas give

$$
\begin{aligned}
u\left(x_{\mathcal{L}^{\prime}}\right)-u\left(x_{\mathcal{L}}\right)= & \left(t_{\mathcal{L}^{\prime}}-t_{\mathbf{v}}\right)(u \circ \varphi)^{\prime}\left(t_{\mathrm{v}}\right)+\int_{t_{\mathbf{v}}}^{t_{\mathcal{L}^{\prime}}}\left(t_{\mathcal{L}^{\prime}}-s\right)(u \circ \varphi)^{\prime \prime}(s) \mathrm{d} s \\
& -\left(t_{\mathcal{L}}-t_{\mathbf{v}}\right)(u \circ \varphi)^{\prime}\left(t_{\mathbf{v}}\right)-\int_{t_{\mathbf{v}}}^{t_{\mathcal{L}}}\left(t_{\mathcal{L}}-s\right)(u \circ \varphi)^{\prime \prime}(s) \mathrm{d} s
\end{aligned}
$$

Noting that $\left|t_{\mathcal{L}^{\prime}}-t_{\mathcal{L}}\right|=m_{\gamma_{\mathcal{L} \mathcal{L}^{\prime}}}$ we conclude the proof.

Thanks to the Taylor's formulas we can prove the following proposition.

Proposition A.2. Let $\mathcal{L} \in \partial \mathfrak{M}$ be a boundary control volume and $\mathbf{v}$ be a vertex of $\mathcal{L}$, then the following equality holds

$$
m_{\gamma_{\mathcal{L} \mathbf{v}}}-d_{\mathcal{L}, \mathbf{v}}=\mathcal{O}\left(m_{\mathcal{L}} m_{\gamma_{\mathcal{L} \mathbf{v}}}\right) .
$$

Moreover for any point $x \in \sigma=\mathcal{L} \in \mathcal{E}_{\text {ext }}$, one has

$$
\overrightarrow{\mathbf{n}}_{\sigma \mathcal{K}}(x)-\overrightarrow{\mathbf{n}}_{\mathcal{K} \mathcal{L}}=\mathcal{O}\left(m_{\mathcal{L}}\right),
$$

where $\overrightarrow{\mathbf{n}}_{\sigma \mathcal{K}}(x)$ is the unit normal vector to $\sigma$ outward to $\mathcal{K}$ at point $x$.

We are now in position to prove the main result of the appendix.

THEOREM A.3. Let us assume that the solution u of the continuous problem (A.1) belongs to $H_{\Gamma}^{2}(\Omega)$. Let us consider the solution $u_{\mathcal{T}}$ to discrete problem (A.2). Then, there exists $C_{27}>0$ independent of $\operatorname{size}(\mathcal{T})$ such that

$$
\left|e_{\mathcal{T}}\right|_{1, \mathcal{T}}^{2}+\left|e_{\partial \mathfrak{M}}\right|_{1, \partial \mathfrak{M}}^{2} \leq C_{27} \operatorname{size}(\mathcal{T})^{2}\|u\|_{H_{\Gamma}^{2}(\Omega)}^{2},
$$

with $e_{\mathcal{T}}=\mathbb{P}_{\mathcal{T}}^{c} u-u_{\mathcal{\tau}}$.

We decompose the proof of Theorem A.3 into two steps. As a first step, we prove (see Proposition A.4) that the left-hand side of inequality (A.4) is bounded from above by the different consistency errors which intervene in the problem. In a second phase, we have to estimate these different consistency errors.

Proposition A.4. Let us consider the solution u to problem (A.1) and the solution $u_{\mathcal{T}}$ to discrete problem (A.2). Then the following estimate holds

$$
\begin{aligned}
\left|e_{\mathcal{T}}\right|_{1, \mathcal{T}}^{2}+\left|e_{\partial \mathfrak{M}}\right|_{1, \partial \mathfrak{M}}^{2} \leq & \sum_{\sigma=\mathcal{K} \mid \mathcal{L} \in \mathcal{E}_{i n t}} m_{\sigma} d_{\mathcal{K}, \mathcal{L}}\left(R_{\sigma, \mathcal{K}}^{i n t}\right)^{2}+\sum_{\sigma=\mathcal{L} \in \mathcal{E}_{\text {ext }}} m_{\mathbf{e}_{\mathcal{L}}} d_{\mathcal{K}, \mathcal{L}}\left(R_{\sigma, \mathcal{K}}^{e x t}\right)^{2} \\
& +\sum_{\mathbf{v}=\mathcal{L} \mid \mathcal{L}^{\prime} \in \mathcal{V}} \frac{R_{\mathbf{v}, \mathcal{L}^{2}}}{d_{\mathcal{L}, \mathcal{L}^{\prime}}}
\end{aligned}
$$

where,

$$
\begin{array}{lll}
R_{\sigma, \mathcal{K}}^{i n t}=\frac{1}{m_{\sigma}} \int_{\sigma} \nabla u(x) \cdot \overrightarrow{\mathbf{n}}_{\mathcal{K} \mathcal{L}} \mathrm{d} x-\frac{u\left(x_{\mathcal{L}}\right)-u\left(x_{\mathcal{K}}\right)}{d_{\mathcal{K}, \mathcal{L}}}, & & \forall \sigma=\mathcal{K} \mid \mathcal{L} \in \mathcal{E}_{\mathcal{K}} \cap \mathcal{E}_{i n t} ; \\
R_{\sigma, \mathcal{K}}^{e x t}=\frac{1}{m_{\mathbf{e}_{\mathcal{L}}}} \int_{\sigma} \nabla u(x) \cdot \overrightarrow{\mathbf{n}}_{\sigma \mathcal{K}}(x) \mathrm{d} x-\frac{u\left(x_{\mathcal{L}}\right)-u\left(x_{\mathcal{K}}\right)}{d_{\mathcal{K}, \mathcal{L}}}, & & \forall \sigma=\mathcal{L} \in \mathcal{E}_{\mathcal{K}} \cap \mathcal{E}_{\text {ext }} ; \\
R_{\mathbf{v}, \mathcal{L}}=d_{\mathcal{L}, \mathcal{L}^{\prime}} \nabla_{\Gamma} u_{\mid \Gamma}(\mathbf{v}) \overrightarrow{\boldsymbol{\tau}}_{\mathbf{v}, \mathcal{L}}-u_{\mid \Gamma}\left(x_{\mathcal{L}^{\prime}}\right)-u_{I \Gamma}\left(x_{\mathcal{L}}\right), & & \forall \mathbf{v}=\mathcal{L} \mid \mathcal{L}^{\prime} \in \mathcal{V} .
\end{array}
$$


Proof. Let $\mathcal{K} \in \mathfrak{M}$, we integrate equation (A.1a) on $\mathcal{K}$ and we subtract the resulting equality with equation (A.2a). Thanks to definitions of $R_{\sigma, \mathcal{K}}^{i n t}$ and $R_{\sigma, \mathcal{K}}^{e x t}$ given in Proposition A.4 imply

$$
\begin{aligned}
\sum_{\sigma=\mathcal{K} \mid \mathcal{L} \in \mathcal{E}_{\text {int }}} \frac{m_{\sigma}}{d_{\mathcal{K}, \mathcal{L}}}\left(e_{\mathcal{K}}-e_{\mathcal{L}}\right) & +\sum_{\sigma=\mathcal{L} \in \mathcal{E}_{\text {ext }}} \frac{m_{\mathbf{e}_{\mathcal{L}}}}{d_{\mathcal{K}, \mathcal{L}}}\left(e_{\mathcal{K}}-e_{\mathcal{L}}\right) \\
& =\sum_{\sigma \in \mathcal{E}_{\mathcal{K}}^{\text {int }}} m_{\sigma} R_{\sigma, \mathcal{K}}^{\text {int }}+\sum_{\sigma \in \mathcal{E}_{\mathcal{K}}^{\text {ext }}} m_{\mathbf{e}_{\mathcal{L}}} R_{\sigma, \mathcal{K}}^{e x t}, \quad \forall \mathcal{K} \in \mathfrak{M} .
\end{aligned}
$$

In the same way let $\mathcal{L} \in \partial \mathfrak{M}$, we integrate equation $(\mathrm{A} .1 \mathrm{~b})$ on $\mathcal{L}$ and we subtract the resulting equality with equation (A.2b). Then we obtain

$$
\sum_{\mathbf{v} \in \mathcal{V}_{\mathcal{L}}} \frac{e_{\mathcal{L}}-e_{\mathcal{L}^{\prime}}}{d_{\mathcal{L}, \mathcal{L}^{\prime}}}+m_{\mathbf{e}_{\mathcal{L}}} \frac{e_{\mathcal{L}}-e_{\mathcal{K}}}{d_{\mathcal{K}, \mathcal{L}}}=\sum_{\mathbf{v} \in \mathcal{V}_{\mathcal{L}}} \frac{R_{\mathbf{v}, \mathcal{L}}}{d_{\mathcal{L}, \mathcal{L}^{\prime}}}-m_{\mathbf{e}_{\mathcal{L}}} R_{\sigma, \mathcal{K}}^{\text {ext }}, \quad \forall \mathcal{L} \in \partial \mathfrak{M} .
$$

Now we multiply equation (A.6) by $e_{\mathcal{K}}$ and summing up over $\mathcal{K} \in \mathfrak{M}$ and we multiply equation $(\mathrm{A} .7)$ by $e_{\mathcal{L}}$ and summing up over $\mathcal{L} \in \partial \mathfrak{M}$. Then, summing the resulting equalities we have

$$
\begin{aligned}
\left|e_{\mathcal{T}}\right|_{1, \mathcal{T}}^{2}+\left|e_{\partial \mathfrak{M}}\right|_{1, \partial \mathfrak{M}}^{2}= & \sum_{\sigma=\mathcal{K} \mid \mathcal{L} \in \mathcal{E}_{i n t}} m_{\sigma}\left(e_{\mathcal{K}}-e_{\mathcal{L}}\right) R_{\sigma, \mathcal{K}}^{i n t}+\sum_{\sigma=\mathcal{L} \in \mathcal{E}_{\text {ext }}} m_{\mathbf{e}_{\mathcal{L}}}\left(e_{\mathcal{K}}-e_{\mathcal{L}}\right) R_{\sigma, \mathcal{K}}^{e x t} \\
& +\sum_{\mathbf{v}=\mathcal{L} \mid \mathcal{L}^{\prime} \in \mathcal{V}} R_{\mathbf{v}, \mathcal{L}} \frac{e_{\mathcal{L}}-e_{\mathcal{L}^{\prime}}}{d_{\mathcal{L}, \mathcal{L}^{\prime}}}
\end{aligned}
$$

Owing to the Cauchy-Schwarz and the Young inequalities, we obtain estimate (A.5).

With this proposition at hand we are now able to prove Theorem A.3 by estimating all the terms of the right-hand side of (A.5).

Proof. First, let $\sigma=\mathcal{K} \mid \mathcal{L} \in \mathcal{E}_{\text {int }}$ thanks to the Taylor's formulas we have

$$
\begin{aligned}
R_{\sigma, \mathcal{K}}^{\text {int }}= & \frac{1}{m_{\sigma} d_{\mathcal{K}, \mathcal{L}}} \int_{\sigma} \int_{0}^{1}(1-t)\left\langle D^{2} u\left((1-t) x+t x_{\mathcal{K}}\right)\left(x_{\mathcal{K}}-x\right),\left(x_{\mathcal{K}}-x\right)\right\rangle \\
& -\frac{1}{m_{\sigma} d_{\mathcal{K}, \mathcal{L}}} \int_{\sigma} \int_{0}^{1}(1-t)\left\langle D^{2} u\left((1-t) x+t x_{\mathcal{L}}\right)\left(x_{\mathcal{L}}-x\right),\left(x_{\mathcal{L}}-x\right)\right\rangle .
\end{aligned}
$$

Owing to the Jensen inequality and the change of variables $(t, x) \in[0,1] \times \sigma \mapsto y=x+t\left(x_{\mathcal{K}}-x\right)$ (or $(t, x) \in[0,1] \times \sigma \mapsto y=x+t\left(x_{\mathcal{L}}-x\right)$ for the second term) and since for any $\mathcal{K} \in \mathfrak{M}$, $\operatorname{diam}(\mathcal{K}) \leq \operatorname{reg}(\mathcal{T}) d\left(x_{\mathcal{K}}, \sigma\right)$, for any $\sigma \in \mathcal{E}_{\mathcal{K}}$ (see Definition 2.1) one has

$$
\left(R_{\sigma, \mathcal{K}}^{\text {int }}\right)^{2} \leq C(\operatorname{reg}(\mathcal{T})) \frac{\operatorname{size}(\mathcal{T})^{2}}{m_{\mathcal{D}}} \int_{\mathcal{D}}\left|D^{2} u(y)\right|^{2} \mathrm{~d} y
$$

Noting that $m_{\mathcal{D}}=\frac{m_{\sigma} d_{\mathcal{K}, \mathcal{L}}}{2}$ we obtain

$$
\sum_{\sigma=\mathcal{K} \mid \mathcal{L} \in \mathcal{E}_{i n t}} m_{\sigma} d_{\mathcal{K}, \mathcal{L}}\left(R_{\sigma, \mathcal{K}}^{i n t}\right)^{2} \leq C(\operatorname{reg}(\mathcal{T})) \operatorname{size}(\mathcal{T})^{2}\left\|D^{2} u\right\|_{L^{2}(\Omega)}^{2}
$$

Secondly let $\sigma=\mathcal{L} \in \partial \mathfrak{M}$, thanks to definition (A.3) of $\widetilde{u}$ we have $u\left(x_{\mathcal{L}}\right)=\widetilde{u}\left(x_{\mathcal{L}}\right)$ and $u\left(x_{\mathcal{K}}\right)=\widetilde{u}\left(x_{\mathcal{K}}\right)$, thus since $x_{\mathcal{L}}-x_{\mathcal{K}}=d\left(x_{\mathcal{K}}, x_{\mathcal{L}}\right) \overrightarrow{\mathbf{n}}_{\mathcal{K} \mathcal{L}}$, the definition of $R_{\sigma, \mathcal{K}}^{e x t}$, the Jensen inequality 
and the Taylor's formulas imply

$$
\begin{aligned}
m_{\mathbf{e}_{\mathcal{L}}} d_{\mathcal{K}, \mathcal{L}}\left(R_{\sigma, \mathcal{K}}^{e x t}\right)^{2} \leq & 5 d_{\mathcal{K}, \mathcal{L}} \frac{\left(m_{\mathcal{L}}-m_{\mathbf{e}_{\mathcal{L}}}\right)^{2}}{m_{\mathbf{e}_{\mathcal{L}}} m_{\mathcal{L}}} \int_{\mathcal{L}}|\nabla \widetilde{u}(x)|^{2} \mathrm{~d} \sigma(x) \\
& +5 \frac{\left(d_{\mathcal{K}, \mathcal{L}}-d\left(x_{\mathcal{K}}, x_{\mathcal{L}}\right)\right)^{2}}{d_{\mathcal{K}, \mathcal{L}}} \frac{m_{\mathbf{e}_{\mathcal{L}}}}{m_{\mathcal{L}}} \int_{\mathcal{L}}|\nabla \widetilde{u}(x)|^{2} \mathrm{~d} \sigma(x) \\
& +5 d_{\mathcal{K}, \mathcal{L}} \frac{m_{\mathbf{e}_{\mathcal{L}}}}{m_{\mathcal{L}}} \int_{\mathcal{L}}|\nabla \widetilde{u}(x)|^{2}\left|\overrightarrow{\mathbf{n}}_{\mathcal{K} \mathcal{L}}-\overrightarrow{\mathbf{n}}_{\sigma \mathcal{K}}(x)\right|^{2} \mathrm{~d} \sigma(x) \\
& +\frac{5 m_{\mathbf{e}_{\mathcal{L}}}}{d_{\mathcal{K}, \mathcal{L}} m_{\sigma}} \int_{\sigma} \int_{0}^{1}(1-t)^{2}\left|D^{2} \widetilde{u}\left((1-t) x+t x_{\mathcal{K}}\right)\right|^{2}\left|x_{\mathcal{K}}-x\right|^{4} \mathrm{~d} t \mathrm{~d} \sigma(x) \\
& +\frac{5 m_{\mathbf{e}_{\mathcal{L}}}}{d_{\mathcal{K}, \mathcal{L}} m_{\sigma}} \int_{\sigma} \int_{0}^{1}(1-t)^{2}\left|D^{2} \widetilde{u}\left((1-t) x+t x_{\mathcal{L}}\right)\right|^{2}\left|x_{\mathcal{L}}-x\right|^{4} \mathrm{~d} t \mathrm{~d} \sigma(x) .
\end{aligned}
$$

Thanks to Propositions 2.2, 2.3 and A.2, there exists $C_{\Gamma}>0$ independent of $\operatorname{size}(\mathcal{T})$ such that

$$
\left|d\left(x_{\mathcal{K}}, x_{\mathcal{L}}\right)-d_{\mathcal{K}, \mathcal{L}}\right| \leq C_{\Gamma} m_{\mathcal{L}} m_{\gamma_{\mathcal{L} \mathbf{v}}}, \quad\left|m_{\mathbf{e}_{\mathcal{L}}}-m_{\mathcal{L}}\right| \leq C_{\Gamma} m_{\mathcal{L}}^{3} \text { and }\left|\overrightarrow{\mathbf{n}}_{\mathcal{K} \mathcal{L}}-\overrightarrow{\mathbf{n}}_{\boldsymbol{\sigma} \mathcal{K}}(x)\right| \leq C_{\Gamma} m_{\mathcal{L}}
$$

Thus, thanks to a change of variables in the last two integrals we have

$$
\begin{aligned}
m_{\mathbf{e}_{\mathcal{L}}} d_{\mathcal{K}, \mathcal{L}}\left(R_{\sigma, \mathcal{K}}^{e x t}\right)^{2} \leq & C_{\Gamma}(\operatorname{reg}(\mathcal{T})) \operatorname{size}(\mathcal{T})^{3} \int_{\mathcal{L}}|\nabla \widetilde{u}(x)|^{2} \mathrm{~d} \sigma(x) \\
& +C(\operatorname{reg}(\mathcal{T})) \operatorname{size}(\mathcal{T})^{2}\left(\int_{\mathcal{D}_{\mathcal{L}}}\left|D^{2} \widetilde{u}(y)\right|^{2} \mathrm{~d} y+\int_{\mathcal{D}}\left|D^{2} \widetilde{u}(y)\right|^{2} \mathrm{~d} y\right),
\end{aligned}
$$

where $\mathcal{D}_{\mathcal{L}}=\left\{(1-t) x+t x_{\mathcal{L}}: t \in[0,1], x \in \sigma=\mathcal{L}\right\}$. Then, owing to (A.3) we obtain

$$
\sum_{\sigma=\mathcal{L} \in \mathcal{E}_{\text {ext }}} m_{\mathbf{e}_{\mathcal{L}}} d_{\mathcal{K}, \mathcal{L}}\left(R_{\sigma, \mathcal{K}}^{e x t}\right)^{2} \leq C_{\Gamma}(\operatorname{reg}(\mathcal{T})) C_{26} \operatorname{size}(\mathcal{T})^{2}\|u\|_{H^{1}(\Omega)}^{2} .
$$

Finally, using definition of $R_{\mathbf{v}, \mathcal{L}}$ for any $\mathbf{v}=\mathcal{L} \mid \mathcal{L}^{\prime} \in \mathcal{V}$ we have

$$
\frac{R_{\mathbf{v}, \mathcal{L}^{2}}^{2}}{d_{\mathcal{L}, \mathcal{L}^{\prime}}} \leq 2 d_{\mathcal{L}, \mathcal{L}^{\prime}}\left(\nabla_{\Gamma} u(\mathbf{v}) \vec{\tau}_{\mathbf{v}, \mathcal{L}}-\frac{u\left(x_{\mathcal{L}^{\prime}}\right)-u\left(x_{\mathcal{L}}\right)}{m_{\gamma_{\mathcal{L} \mathcal{L}^{\prime}}}}\right)^{2}+2 \frac{\left(u\left(x_{\mathcal{L}^{\prime}}\right)-u\left(x_{\mathcal{L}}\right)\right)^{2}}{m_{\gamma_{\mathcal{L} \mathcal{L}^{\prime}}}} \frac{\left(d_{\mathcal{L}, \mathcal{L}^{\prime}}-m_{\gamma_{\mathcal{L} \mathcal{L}^{\prime}}}\right)^{2}}{d_{\mathcal{L}, \mathcal{L}^{\prime}} m_{\gamma_{\mathcal{L} \mathcal{L}^{\prime}}}}
$$

Thanks to Proposition A.1 and A.2, we obtain

$$
\sum_{\mathbf{v}=\mathcal{L} \mid \mathcal{L}^{\prime} \in \mathcal{V}} \frac{R_{\mathbf{v}, \mathcal{L}}^{2}}{d_{\mathcal{L}, \mathcal{L}^{\prime}}} \leq 2 C_{\Gamma} \operatorname{size}(\mathcal{T})^{2}\left\|u_{I \Gamma}\right\|_{H^{2}(\Gamma)}^{2}
$$

Gathering estimates (A.8), (A.9) and (A.10) the claim follows.

We have obtained an error estimate between the center-value projection of the exact solution $\mathbb{P}_{\mathcal{T}}^{c} u$ and the approximate solution $u_{\mathcal{T}}$ for the Laplace problem with Ventcell boundary conditions for the $H^{1}$-seminorms. However in order to prove Proposition 4.11 we also need to prove an estimate between the exact solution $u$ and the approximate solution $u_{\mathcal{T}}$ for the $L^{2}$-norms. To conclude we adopt here the same reasoning as that given in [11, Theorem 10.1] for the Laplace problem with Neumann boundary conditions, apart from the fact that here the domain is not polygonal.

Let $\beta_{\mathcal{T}} \in \mathbb{R}$ such that $\sum_{\mathcal{K} \in \mathfrak{M}} m_{\underline{\mathcal{K}}} \bar{u}\left(x_{\mathcal{K}}\right)=\alpha$ with $\bar{u}=u+\beta_{\mathcal{T}}$. Setting $\bar{e}_{\mathcal{K}}=\bar{u}\left(x_{\mathcal{K}}\right)-u_{\mathcal{K}}$ for any $\mathcal{K} \in \mathfrak{M}$ and $\bar{e}_{\mathcal{L}}=\bar{u}\left(x_{\mathcal{L}}\right)-\bar{u}_{\mathcal{L}}$ for any $\mathcal{L} \in \partial \mathfrak{M}$, estimate (A.4) is also satisfied for 
$\bar{e}_{\mathcal{T}}$. However, thanks to its definition the error $\bar{e}_{\mathfrak{M}}$ has now zero mean-value on $\underline{\Omega}$. Thus if $\operatorname{size}(\mathcal{T}) \leq \frac{1}{2 C_{5}}$, the discrete Poincaré inequality $(2.3)$ gives

$$
\left\|\bar{e}_{\mathfrak{M}}\right\|_{L^{2}(\Omega)}^{2} \leq 4 C_{4}^{2} C_{27} \operatorname{size}(\mathcal{T})^{2}\|u\|_{H_{\Gamma}^{2}(\Omega)}^{2},
$$

and thanks to the trace inequality (Lemma 2.10) we have

$$
\left\|\bar{e}_{\partial \mathfrak{M}}\right\|_{L^{2}(\Gamma)}^{2} \leq\left(1+2 C_{4}\right)^{2} C_{8}^{2} C_{27} \operatorname{size}(\mathcal{T})^{2}\|u\|_{H_{\Gamma}^{2}(\Omega)}^{2} .
$$

Thanks to the regularity of the function $u$, denoting by $L_{u}$ the Lipschitz constant of $u$, one has

$$
\left\|u-u_{\mathcal{T}}\right\|_{L^{2}(\Omega)}^{2} \leq 3|\Omega| L_{u}^{2} \operatorname{size}(\mathcal{T})^{2}+3|\Omega| \beta_{\mathcal{T}}^{2}+3\left\|\bar{e}_{\mathfrak{M}}\right\|_{L^{2}(\Omega)}^{2} .
$$

We recall that $\int_{\Omega} u=\sum_{\mathcal{K} \in \mathfrak{M}} m_{\underline{\mathcal{K}}} \bar{u}\left(x_{\mathcal{K}}\right)=\alpha$ and $\beta_{\mathcal{T}}=\bar{u}-u$, thus one has

$$
|\underline{\Omega}| \beta_{\mathcal{T}}=\alpha-\sum_{\mathcal{K} \in \mathfrak{M}} m_{\underline{\mathcal{K}}} u\left(x_{\mathcal{K}}\right)
$$

and

$$
\sum_{\mathcal{K} \in \mathfrak{M}} m_{\underline{\mathcal{K}}} u\left(x_{\mathcal{K}}\right)=\sum_{\mathcal{K} \in \mathfrak{M}}\left(m_{\underline{\mathcal{K}}}-m_{\mathcal{K}}\right) u\left(x_{\mathcal{K}}\right)+\sum_{\mathcal{K} \in \mathfrak{M}} \int_{\mathcal{K}}\left(u\left(x_{\mathcal{K}}\right)-u(x)\right)+\alpha .
$$

Thus thanks to the regularity of $u$, Proposition 2.1 and the mesh regularity (2.1) we can claim that $\left|\beta_{\mathcal{T}}\right| \leq C \operatorname{size}(\mathcal{T})$ that concludes the proof.

The reasoning is exactly the same for the $L^{2}(\Gamma)$-norm that concludes the claim.

\section{REFERENCES}

[1] M. Bessemoulin-Chatard, C. Chainais-Hillairet, and F. Filbet, On discrete functional inequalities for some finite volume schemes, IMA J. Numer. Anal., 35 (2015), pp. 1125-1149, https://doi.org/ https://doi.org/10.1093/imanum/dru032.

[2] L. Cherfils, M. Petcu, and M. Pierre, A numerical analysis of the Cahn-Hilliard equation with dynamic boundary conditions, Discrete Contin. Dyn. Syst., 27 (2010), pp. 1511-1533, https://doi. org/https://doi.org/10.3934/dcds.2010.27.1511.

[3] S.-M. Сhoo, S.-K. Chung, And K.-I. Kim, Conservative nonlinear difference scheme for the CahnHilliard equation-II, Computers and Mathematics with Applications, 39 (2000), pp. 229 - 243, https://doi.org/https://doi.org/10.1016/S0898-1221(99)00326-0.

[4] J. Droniou and N. Nataraj, Improved $L^{2}$ estimate for gradient schemes and super-convergence of the TPFA finite volume scheme, IMA J. Numer. Anal., 38 (2017), pp. 1254-1293, https://doi.org/https: //doi.org/10.1093/imanum/drx028.

[5] Q. Du And R.-A. Nicolaides, Numerical analysis of a continuum model of phase transition, SIAM J. Numer. Anal., 28 (1991), pp. 1310-1322, https://doi.org/https://doi.org/10.1137/0728069.

[6] C.-M. ElLiott, The Cahn-Hilliard model for the kinetics of phase separation, in Mathematical models for phase change problems (Óbidos, 1988), vol. 88 of Internat. Ser. Numer. Math., Birkhäuser, Basel, 1989, pp. 35-73, https://doi.org/https://doi.org/10.1007/978-3-0348-9148-6_3.

[7] C.-M. Elliott and D.-A. French, Numerical studies of the Cahn-Hilliard equation for phase separation, IMA J. Appl. Math., 38 (1987), pp. 97-128, https://doi.org/https://doi.org/10.1093/imamat/ 38.2.97.

[8] C.-M. Elliott And D.-A. French, A nonconforming finite-element method for the two-dimensional Cahn-Hilliard equation, SIAM J. Numer. Anal., 26 (1989), pp. 884-903, https://doi.org/https://doi. org $/ 10.1137 / 0726049$.

[9] C.-M. Elliott, D.-A. French, and F.-A. Milner, A second order splitting method for the CahnHilliard equation, Numer. Math., 54 (1989), pp. 575-590, https://doi.org/https://doi.org/10.1007/ BF01396363.

[10] C.-M. Elliott And S. Larsson, Error estimates with smooth and nonsmooth data for a finite element method for the Cahn-Hilliard equation, Math. Comp., 58 (1992), pp. 603-630, https://doi.org/https: //doi.org/10.2307/2153205. 
[11] R. Eymard, T. Gallouët, and R. Herbin, Finite volume methods, in Handbook of numerical analysis, Vol. VII, P. Ciarlet and J. Lions, eds., Handb. Numer. Anal., VII, North-Holland, Amsterdam, 2000, pp. $715-1022$.

[12] D.-J. EyRE, Unconditionally gradient stable time marching the Cahn-Hilliard equation, in Symposia BB Computational and Mathematical Models of Microstructural Evolution, vol. 529 of MRS Proceedings, 1 1998, https://doi.org/https://doi.org/10.1557/PROC-529-39.

[13] X. Feng and A. Prohl, Error analysis of a mixed finite element method for the Cahn-Hilliard equation, Numer. Math., 99 (2004), pp. 47-84, https://doi.org/https://doi.org/10.1007/s00211-004-0546-5.

[14] X. Feng And A. Prohl, Numerical analysis of the Cahn-Hilliard equation and approximation of the Hele-Shaw problem, Interfaces Free Bound., 7 (2005), pp. 1-28, https://doi.org/https://doi.org/10. 4171/IFB/111.

[15] H. Fischer, P. MaAss, And W. Dieterich, Novel surface modes in spinodal decomposition, Phys. Rev. Lett., 79 (1997), pp. 893-896, https://doi.org/https://doi.org/10.1103/PhysRevLett.79.893.

[16] H. Fischer, P. MaAss, And W. Dieterich, Diverging time and length scales of spinodal decomposition modes in thin films, EPL (Europhysics Letters), 42 (1998), pp. 49-54, https://doi.org/https://doi. org/10.1209/epl/i1998-00550-y.

[17] D. Furihata, A stable and conservative finite difference scheme for the Cahn-Hilliard equation, Numer. Math., 87 (2001), pp. 675-699, https://doi.org/https://doi.org/10.1007/PL00005429.

[18] D. JAcqmin, Contact-line dynamics of a diffuse fluid interface, J. Fluid Mech., 402 (2000), pp. 57-88, https://doi.org/https://doi.org/10.1017/S0022112099006874.

[19] D. Kay, V. Styles, and R. Welford, Finite element approximation of a Cahn-Hilliard-Navier-Stokes system, Interfaces Free Bound., 10 (2008), pp. 15-43, https://doi.org/https://doi.org/10.4171/IFB/ 178.

[20] R. Kenzler, F. Eurich, P. Maass, B. Rinn, J. Schropp, E. Bohl, and W. Dieterich, Phase separation in confined geometries: Solving the Cahn-Hilliard equation with generic boundary conditions, j-Comp-Phys-Comm, 133 (2001), pp. 139-157, https://doi.org/https://doi.org/10.1016/ S0010-4655(00)00159-4.

[21] B. Kovács And C. Lubich, Numerical analysis of parabolic problems with dynamic boundary conditions, IMA J. Numer. Anal., 37 (2016), pp. 1-39, https://doi.org/https://doi.org/10.1093/imanum/drw015.

[22] F. NABEt, Convergence of a finite-volume scheme for the Cahn-Hilliard equation with dynamic boundary conditions, IMA J. Numer. Anal., 36 (2016), pp. 1898-1942, https://doi.org/https://doi.org/10.1093/ imanum/drv057.

[23] J. Shen And X. YAng, Numerical approximations of Allen-Cahn and Cahn-Hilliard equations, Discrete Contin. Dyn. Syst., 28 (2010), pp. 1669-1691, https://doi.org/https://doi.org/10.3934/dcds.2010.28. 1669. 